\title{
Molecular Beam Surface Analysis 1993 Summary Report
}

A. D. Appelhans, J. C. Ingram, G. S. Groenewold, D. A. Dahl and J. E. Delmore

Published September 1993

EG\&G Idaho, Inc.

Idaho Falls, Idaho 83415

Prepared for the

U.S. Department of Energy

Idaho Operations Office

Under DOE Contract No. DE-AC07-76IDO1570 


\section{Contents}

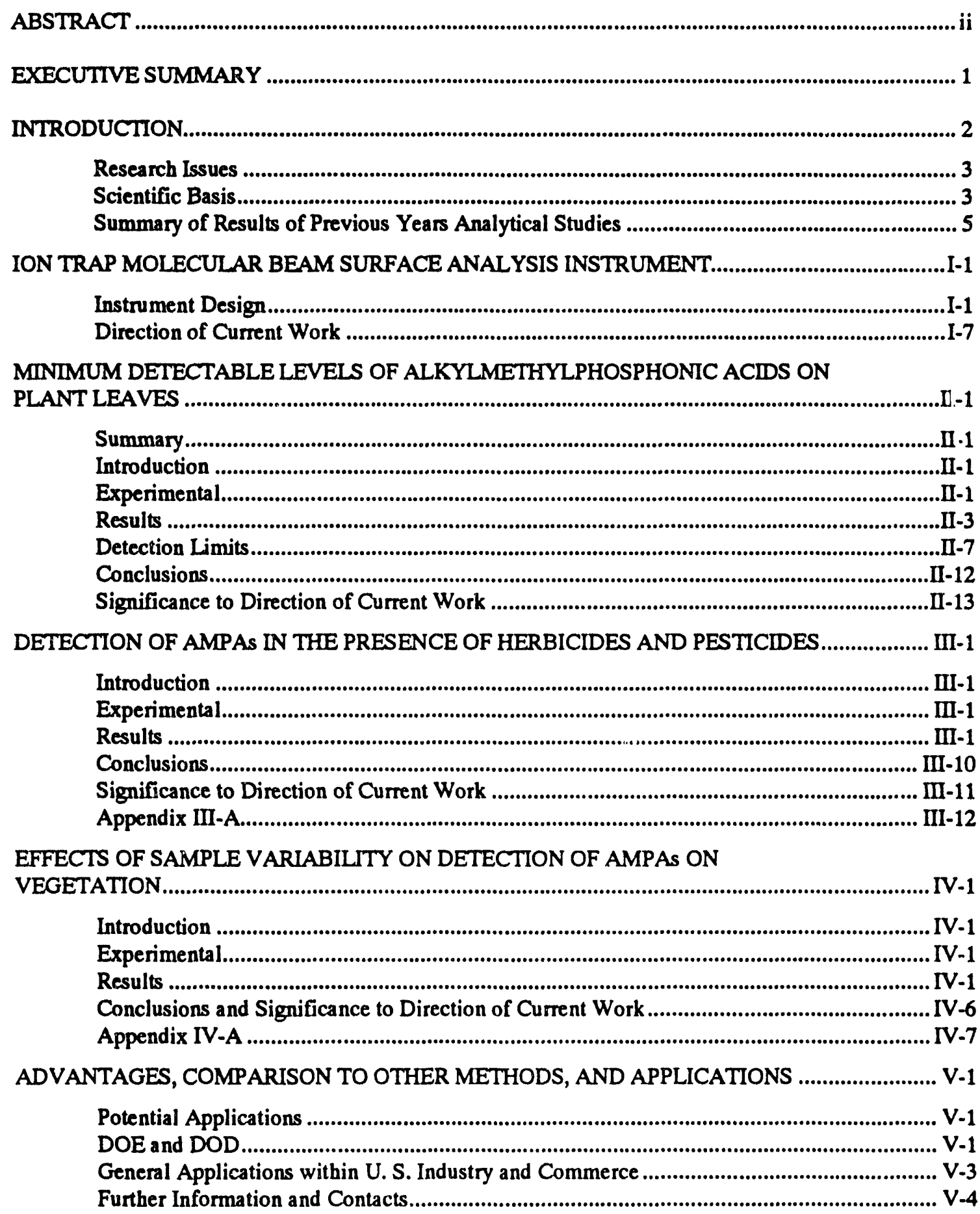




\begin{abstract}
The Molecular Beam Surface Analysis (MBSA) program is developing both laboratory-based and potentially field-portable chemical anaiyses systems taking advantage of new surface analysis technology developed at the Idaho National Engineering Laboratory (INEL). The objective is to develop the means to rapidly detect and identify, with high specificity and high sensitivity, nonvolatile and low volatile organics found in Chemical Weapons (CW) and High Explosives (HE) feedstocks, agents, and decomposition products on surfaces of plants, rocks, paint chips, filters, smears of buildings, vehicles, equipment, etc.. Ideally, the method would involve no sample preparation and no waste generation, and would have the potential for being implemented as a field-portable instrument. In contrast to existing analytical methods that rely on sample volatility, MBSA is optimized for nonvolatile and low volatile compounds. This makes it amenable for rapidly screening field samples for $\mathrm{CW}$ agent decomposition products and feedstock chemicals and perhaps actual agents. In its final configuration (benchtop size) it could be operated in a non-laboratory environment (such as an office building) requiring no sample preparation chemistry or chemical supplies. It could also be included in a mobile laboratory used in on-site, or remote site cooperative surveys, or in a standard laboratory, where it would provide fast screening of samples at minimal cost.
\end{abstract}




\title{
Molecular Beam Surface Analysis 1993 Summary Report
}

\author{
A. D. Appelhans, J. C. Ingram, G. S. Groenewold, D. A. Dahl and J. E. Delmore \\ Idaho National Engineering Laboratory
}

\section{Executive Summary}

The Molecular Beam Surface Analysis (MBSA) program is developing both laboratory-based and potentially field-portable chemical analyses systems taking advantage of new surface analysis technology developed at the Idaho National Engineering Laboratory (INEL). The objective is to develop the means to rapidly detect and identify, with high specificity and high sensitivity, nonvolatile and low volatile organics found in Chemical Weapons (CW) and High Explosives (HE) feedstocks, agents, and decomposition products on surfaces of plants, rocks, paint chips, filters, smears of buildings, vehicles, equipment, etc.. Ideally, the method would involve no sample preparation and no waste generation, and would bave the potential for being implemented as a field-portable instrument. In contrast to existing analytical methods that rely on sample volatility, MBSA is optimized for nonvolatile and low volatile compounds. This makes it amenable for rapidly screening field samples for the low volatility $\mathrm{CW}$ agent decomposition products and feedstock chemicals and, perhaps, actual agents. In its final configuration (benchtop size) it could be operated in a non-laboratory environment (such as an office building) requiring no sample preparation chemistry or chemical supplies. It could also be included in a mobile laboratory used in on-site, or remote site cooperative surveys, or in a standard laboratory, where it would provide fast screening of samples at minimal cost.

In the last two years the scope of the program has included developing an ion trap mass spectrometer based MBSA instrument and conducting a series of analytical studies to demonstrate the viability of the method, the detection levels possible, and evaluate the potential for chemical interferences from environmental sources (herbicides/pesticides). The bulk of the analytical studies have been conducted on a quadrupole mass spectrometer molecular beam surface analysis instrument. The ion trap based instrument is in the prototype stage, and has been available only a very limited time for analytical studies. The prototype instrument includes a fully operational molecular ion gun coupled to a modified ion trap mass spectrometer retrofitted with a unique offset detector system and a sample probe for introducing samples. Initial studies have proven the design of the offset detector system for detecting both positive and negative ions, have established requirements for sample positioning, and demonstrated that the collinear design is successful. Scoping experiments have been performed demonstrating that electrically non conducting samples such as teflon gaskets can be successfully analyzed. In addition, mass spectrometry/mass spectrometry measuriments bave been conducted using CW degradation products, demonstrating both the viability of the method, and the fact that these compounds are amenable to MS/MS a nalysis. These results have provided strong evidence that the specificity of this method will be very good, meeting the goal of reducing to very low probability the chances of false positives during an analysis.

The analytical studies have shown that the general technique is capable of detecting nanogram levels of $\mathrm{CW}$ degradation products on a wide variety of surfaces (vegetation, steels, polymers, rocks etc.), while meeting the goal of no sample preparation chemistry prior to analysis. In addition, it has been demonstrated that the $\mathrm{CW}$ degradation products are detectable in the presence of environmental interferents such as pesticides and herbicides, even when the interferents are present at much higher levels (5 to 10 times). They have also shown that the analysis of $\mathrm{CW}$ degradation products on plant leaves is relatively insensitive to the type of plant chosen. Anecdotal studies have shown that this technique is extremely sensitive for detecting very low levels of contaminants in air by exposing selective surfaces to the air and then examining those surfaces via MBSA. We have been able to detect levels of 
cyclohexylamine in air that were below detection levels of standard techniques such as GCMS. In addition we have been able to directly detect selected $\mathrm{CW}$ degradation products on concrete. In a study conducted for the DOE Office of Waste Management we have shown that low levels of compounds such as tributyl phosphate that are used in nuclear fuel processing can be readily detected on rocks and soils, demonstrating the possible application to nuclear non-proliferation issues.

The general results of the instrument development efforts and the analytical studies indicate there is a high probability that molecular beam surface analysis can meet the goals established at the outset of this program: to develop the means to rapidly detect and identify, with high specificity and high sensitivity, nonvolatile and low volatile organics on surfaces of plants, rocks, paint chips, filters, etc. requiring no sample preparation and no waste generation, and with the potential for being implemented as a fieldportable instrument.

\section{INTRODUCTION}

This report includes an introduction summarizing the overall program and previous testing results followed by five sections dealing in detail with the following topics: I) instrument development; II) evaluation of the sensitivity of MBSA; III) evaluation of chemical interferences; IV) effects of sample variability and anecdotal experiments; and $V$ ) advantages and potential applications of MBSA.

Molecular Beam Surface Analysis is a derivative of Secondary Ion Mass Spectrometry (SIMS) in which a high energy beam of molecular ions is used to bombard the surface of the sample to be analyzed, knocking off molecules (secondary ions) from the sample surface so they can be directed into a mass spectrometer for identification. This is illustrated in Figure 1. Molecular Beam Surfaca Analysis (MBSA) has several significant advantages:

1. It can detect low volatility compounds that are difficult or impossible to detect with gas-phase techniques.

2. It can be applied to an extremely wide range of samples -- essentially any material that is vacuum compatible -- such as vegetation, paint chips, gaskets, filters.

3. It requires no sample preparation other than cutting the sample to the appropriate size $(\sim 1 \mathrm{~cm})$; no chemical extraction, no chromatography, no heating.

4. The analysis typically takes less than 5 minutes.

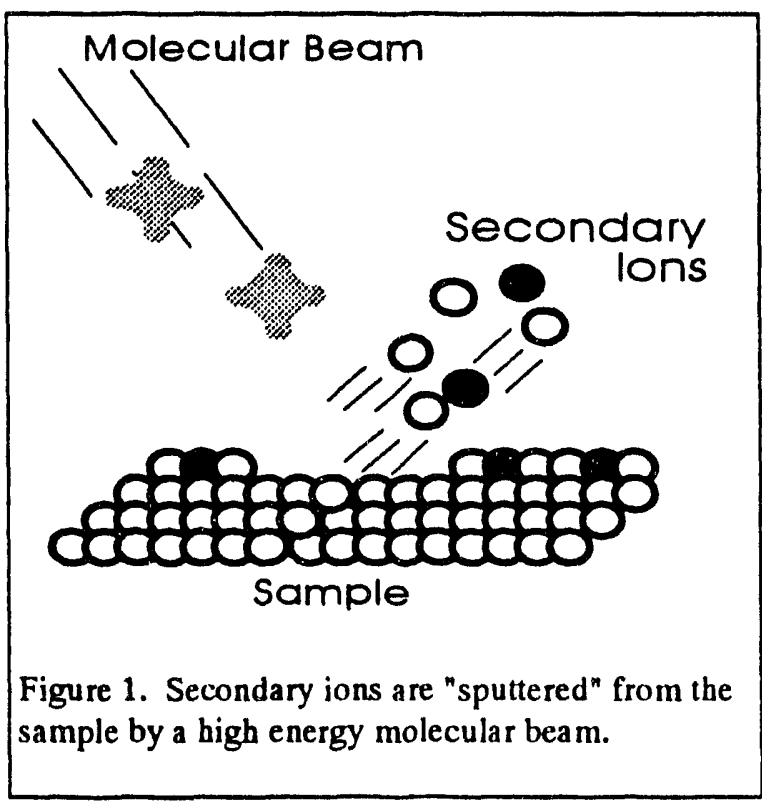

5. There is no waste generated in the sample preparation or analysis, other than the sample itself.

6. It is non-destructive to the bulk of the sample -. only the top few molecular layers are disturbed during the analysis -- so other analytical methods can be applied to the sample following MBSA analysis if desired.

Using a surface analytical method (as opposed to bulk methods) is important because CW agents and related compounds can rapidly decompose in the environment. The decomposition products have very low volatility, and will be adsorbed onto surfaces, which makes direct detection (i.e., no sample 
preparation) difficult or impossible using techniques such as IR, GC, MS, IMS or GC/MS. This difficulty arises because these more conventional techniques require the analyte to be in the gas phase for detection. In contrast, MBSA requires the analyte be condensed on the surface of the sample for detection. Since many of the analyte molecules of interest to the Office of Arms Control will be adsorbed onto the sample surface, MBSA has a distinct advantage when surveying for these analytes.

Two new technological breakthroughs in Secondary Ion Mass Spectrometry instrumentation developed in our DOE Basic Energy Sciences (BES) and Office of Health and Environmental Research (OHER) funded programs provide the foundation for this program. These breakthroughs are: 1) The development of an ion gun that produces a beam of large, high energy negative molecular ions which are highly efficient for "sputtering" charged particles from the surface of materials, and, 2) A pulsed extraction system that alternately draws positive and negative secondary particles from the sample surface allowing electrical insulators such as plant tissue, rocks, gaskets, and ceramic glass-fiber filters to be analyzed directly with no sample preparation. A quadrupole SIMS instrument which incorporates these breakthroughs was developed at the INEL and used to analyze a wide variety of materials (e.g., paint chips, polymers, leaves) for trace contaminants. The success of this technique has led us to explore development of an instrument based on these technologies that could be made small enough to be fieldable, and at the same time provide a highly sensitive and specific capability for detecting low volatility chemicals associated with $\mathrm{CW}$ agents. In our prior studies with the single quadrupole mass spectrometer the instrument performed well in controlled, laboratory conditions, with sensitivities at the nanogram level. However, we anticipate that to provide the required chemical specificity in the presence of an unknown and complex background signal, it will be necessary to achieve better sensitivity and selectivity. We anticipate that these improvements can be achieved using mass spectrometry/mass spectrometry (MS/MS). The new MBSA instrument utilizes an ion trap mass spectrometer, which can perform MS/MS analyses and has other significant advantages over the quadrupole-based systems. The following provides a summary of the research issues, the basis for our approach, and a summary of the results of the analytical studies previously reported.

\section{Research Issues:}

There are two major issues that must be addressed to define the practicability of a fieldable MBSA instrument for application to chemical detection and identification. The first is to demonstrate that MBSA technology can detect chemicals representative of CW-agent feedstocks and degradation products. The degradation products were deposited onto surfaces of samples likely to be encountered in the field: leaves, rocks, gaskets, steel, building materials, etc., and analyzed using the quadrupole SIMS with the ceramic ion gun and pulsed extraction. These measurements provided information on sensitivity, potential false positive/false negative problems, and the chemical behavior of the various sample substrates and compounds. The details of these analyses are discussed further in the later sections of this report.

The second issue is related to the development of the ion trap mass spectrometer based instrument. The goal is to produce a small laboratory version of this instrument which: a) can demonstrate the potential for field applications; and b) can perform MS/MS a nalyses. The primary area of uncertainty related to this issue is how efficiently the ions can be injected into the ion trap. There are currently no commercial SIMS instruments using ion trap technology. Thus the major challenge will be developing ion optics and operating protocols that optimize the ion injection efficiency. This effort will produce a laboratory prototype of the fieldable instrument, it is anticipated that further engineering development necessary to actually take the instrument into the field will be the responsibility of a follow-on program tied directly to an end user.

\section{Scientific Basis:}

In SIMS a high energy beam of atomic ions is directed at the sample, ejecting (sputtering) the analyte as secondary ions from the surface of the sample. The secondary ions are then mass analyzed to identify 
their chemical composition. This significantly reduces or completely eliminates the need for preprocessing of samples (as is necessary for Gas Chromatography-Mass Spectrometry and most other techniques), and addresses a different suite of target chemicals: nonvolatile and low volatiles. SIMS has historically been the most sensitive technique for detecting the presence of materials on the surface of a sample. Both the chemical range and sensitivity of SIMS are excellent. Two new advances in secondary ion mass spectrometry bave been developed at the INEL which expand its range of application to samples relevant to DOE problems: 1) A molecular negative ion beam for bombarding the sample surface has been developed which results in significant increases in sensitivity (30 times) for organic chemicais (see Analytical Chemistry 61 (10) 1087-1093, 1989); and 2) A patented pulsed secondary ion extraction method (see Analytical Chemistry 62 (15) 1679-1686, 1990) has been developed that permits any type of material (vacuum compatible) to be analyzed (even bighly non-conducting samples) and provides simultaneously both the positive and negative secondary ion spectra. These advances make it possible to analyze a raw sample in 5 minutes at sensitivities capable of detecting one monolayer equivalent on a plant leaf surface.

An obvious question arises: Can these types of analyses already be performed on commercial mass spectrometers? Since the state-of-the-art in SIMS is relatively advanced there do indeed exist laboratory based SIMS instruments that can analyze essentially any type of solid sample. The current cadre of commercial time-of-flight (TOF) SIMS instruments offer outstanding sensitivity and mass resolution. However, these are laboratory-based instruments, the inherent design of both the linear and the multiplesector (Charles Evans) types is unsuited to reduction in size, and the instruments can not perform MS/MS measurements (discussed in detail later in this report). The cost of such systems is $\sim \$ 750 \mathrm{k}$. Thus it is impractical to try to develop a rugged, low cost, fieldable unit based on this technology. Magnetic sector based SIMS instruments can provide MS/MS capability and bigh specificity, but are totally unsuited for field use because of their large bulk and power requirements. A third altemative in commercial SIMS technology is triple-quadrupole systems. These can perform MS/MS measurements and thus provide a high degree of specificity and confidence in accurate chemical identification. However they also suffer from size constraints, they are not inherently rugged or robust, and they suffer from limited sensitivity. Advances in the ion trap mass spectrometer over the last few years appear to make it an ideal choice for this application. It is now possible to perform high resolution MS/MS with the ion trap, providing excellent specificity. In addition the ion trap is a small, inherently ngged device well suited to field application, in fact, fieldable ion trap based GC/MS instruments bave been built at Oak Ridge National Laboratory (ORNL) and the U. S. Army's Environmental Research and Development Engineering Center (ERDEC). Ion traps bave been successfully coupled to external ion sources in research instruments, and thus we feel there is a high probability that they can be adapted to the requirements of this program with excellent results. However, there are no commercially available ion trap based secondary ion mass spectrometers. Thus there is a technology gap, and the molecular beam surface analysis instrument is being designed to fill that gap.

In addition to the mass spectrometer it is also important to consider the primary ion gun and the method for sample neutralization (since many field samples are expected to be insulators). Current SIMS instruments utilize either a gas or a solid-state ion gun. Both produce atomic ion beams $\left(\mathrm{Ar}^{+}\right.$or $\mathrm{Xe}^{+}, \mathrm{Cs}^{+}$ or $\mathrm{Ga}^{+}$), which have lower sputtering efficiency than molecular ion beams $\left(\mathrm{ReO}_{4}^{-}\right.$in the $\left.\mathrm{MBSA}\right)$, and both require an electron flood gun to overcome sample charging when analyzing insulating materiais. The gas guns require a gas supply and add significant pumping load to the system. These problems are overcome in the MBSA instrument by using a ceramic-based, molecular ion source ( $\mathrm{ReO} 4^{-}$) and the pulsed extraction sample neutralization method. The ceramic, molecular ion source increases the system sensitivity and is inherently rugged, produces no gas loading, and can be made very compact; ideal for field applications. The pulsed extraction charge neutralization system makes it possible to analyze any insulating material with SIMS without charging problems, and negates the need for electron flooding. Thus system complexity is reduced, both in the number of components in the system (no electron gun) and in its operation. The combination of the molecular beam ion source and the ion trap mass spectrometer 
using pulsed secondary ion sample neutralization now makes it possible to produce a small, fieldable, highly sensitive and selective instrument.

\section{Summary of Results of Previous Years Analytical Studies}

The salient problem with detecting $\mathrm{CW}$ agents which have been weathered in the environment is that the agents undergo degradation reactions (Table 1), that produce products which are characterized by low volatility, and have the capacity for acid-base reactions with surfaces. The analysis of compounds having these characteristics usually requires wet chemical extraction, separation, concentration and derivatization prior to analyses: these steps are costly in terms of time of analysis, expertise and logistics. Analyses requiring these steps are usually not performed in the field because of these limitations.

Table 1. Agents, Degradation Products, and Acronyms

\begin{tabular}{|l|l|l|l|l|}
\hline Agent & $\begin{array}{l}\text { Primary degradation } \\
\text { product }\end{array}$ & Acronym & $\begin{array}{l}\text { Secondary degradation } \\
\text { product }\end{array}$ & Acronym \\
\hline VX & $\begin{array}{l}\text { Ethyl methyl } \\
\text { phosphonic acid }\end{array}$ & EMPA & Methyl phosphonic acid & MPA \\
\hline GB (sarin) & $\begin{array}{l}\text { Isopropyl methyl } \\
\text { phosphonic acid }\end{array}$ & IMPA & Methyl phosphonic acid & MPA \\
\hline GD (soman) & $\begin{array}{l}\text { Pinacolyl methyl } \\
\text { phosphonic acid }\end{array}$ & PMPA & Methyl phosphonic acid & MPA \\
\hline HD (mustard) & Thiodiglycol & TDG & Thiodiglycol sulfoxide & TDGO \\
\hline
\end{tabular}

Molecular beam surface analysis offers the promise of being able to analyze surfaces directly for lowvolatile degradation products, without the need for wet chemical manipulation. In order to demonstrate that relevant $\mathrm{CW}$ degradation products could be detected, to bound the sensitivity of the method, and to explore the possible effects of chemical interactions on the detectability, a series of analytical studies were conducted using actual $\mathrm{CW}$ degradation products on a range of samples that could be encountered in the field.

Chemical warfare agent degradation products were analyzed using an existing, quadrupole-based, molecular beam surface analysis instrument, in order to assess the efficacy of the technique for detection of these chemicals on surfaces of samples. The degradation products EMPA, IMPA, PMPA, MPA, TDG, and TDGO (see Table 1) were analyzed by spiking aqueous solutions of the chemicals onto a variety of materials. Samples analyzed included plant leaves, O-rings, small rocks, paint chips, floor tile, ceiling tile, and stainless steel. These samples were chosen because they may represent the type of sample which might easily be collected as part of a treaty verification or nonproliferation inspection, and they provide a significant analytical challenge. The samples were attached to a target planchette using double-sided tape.

Several trends were identified based on the results of the scoping studies. Among the compounds studied, the alkyl methyl phosphonic acids were the most amenable to SIMS. MPA and TDGO were detected, but with somewhat more difficulty, and TDG could only be detected if larger quantities (ca. $100 \mathrm{ug}$ ) were present (so that the analyte did not completely evaporate in the vacuum system before analysis was complete). The experiments also showed that dandelion leaves, Viton O-rings and steel are good surfaces for detecting the decomposition products. The compounds were also detected on paint chips, rocks and glass, albeit with more difficulty. Detection was more difficult or not possible for samples having less polar surfaces (Teflon, some plant leaves) to which the analytes could not adhere, or porous surfaces (floor and ceiling tile). The primary problem with the porous samples appeared to be that the manner in which the analytes were spiked onto the samples, as solutions in water, tended to exacerbate the absorption into the material. If the analytes had been deposited as a vapor that condensed onto the surface, or as condensates on dust particles that settled onto the surface, we expect they would bave been more easily detected. We felt that these results were particularly promising because few pains were taken to optimize 
the performance of the instrument, each set of spectra were acquired in a single run (no signal averaging), and the initial detectable amounts were in the tens of nanogram range. It was clear that the need exists to better understand the surface chemistry of leaves, polymers and refractory materials in order to optimize sampling and detection strategies. The overall results of these earlier scoping studies indicated that there was significant promise that molecular beam surface analysis could play a role in meeting the need for methods of detecting these types of compounds on a wide variety of surfaces. Table 2 summarizes the results of the scoping studies.

\section{Table 2. Summary of Previous Years Scoping Study Measurements}

\begin{tabular}{|c|c|c|c|}
\hline Sample & Degradation Product & Detected? & Comments \\
\hline Dandelion & PMPA & YES & {$[\mathrm{M}-\mathrm{H}]^{-}$observed } \\
\hline Dandelion & IMPA & YES & {$[\mathrm{M}-\mathrm{H}]^{-}$observed } \\
\hline Dandelion & EMPA & YES & {$[\mathrm{M}-\mathrm{H}]^{-}$observed } \\
\hline Dandelion & MPA & YES & {$[\mathrm{M}-\mathrm{H}]^{-}$observed } \\
\hline Dandelion & TDGO & YES & {$[\mathrm{M}+\mathrm{H}]^{+}$observed } \\
\hline Dandelion & TDG & NO & analyte evaporates \\
\hline Viton O-ring & PMPA & YES & {$[\mathrm{M}-\mathrm{H}]^{-}$observed } \\
\hline Viton O-ring & IMPA & YES & {$[\mathrm{M}-\mathrm{H}]^{-}$observed } \\
\hline Viton O-ring & EMPA & YES & {$[\mathrm{M}-\mathrm{H}]^{-}$observed } \\
\hline Viton O-ring & MPA & YES & {$[\mathrm{M}-\mathrm{H}]^{-}$observed } \\
\hline Viton O-ring & TDGO & YES & {$[\mathrm{M}+\mathrm{H}]^{+}$observed } \\
\hline Viton O-ring & TDG & YES, but & $\begin{array}{l}\text { high evaporation; } \\
{[\mathrm{M}+\mathrm{H}]^{+} \text {observed }}\end{array}$ \\
\hline Paint chip & PMPA & YES & {$[\mathrm{M}-\mathrm{H}]^{-}$observed } \\
\hline Paint chip & IMPA & YES & {$[\mathrm{M}-\mathrm{H}]^{-}$not observed } \\
\hline Paint chip & EMPA & YES & {$[\mathrm{M}-\mathrm{H}]^{-}$not observed } \\
\hline Paint chip & MPA & YES & {$[\mathrm{M}-\mathrm{H}]^{-}$not observed } \\
\hline Stainless steel & PMPA & YES & {$[\mathrm{M}-\mathrm{H}]^{-}$observed } \\
\hline Stainless steel & IMPA & YES & {$[\mathrm{M}-\mathrm{H}]^{-}$observed } \\
\hline Stainless steel & EMPA & YES & {$[\mathrm{M}-\mathrm{H}]^{-}$observed } \\
\hline Stainless steel & MPA & YES & {$[\mathrm{M}-\mathrm{H}]^{-}$observed } \\
\hline Small rock & PMPA & YES & {$[\mathrm{M}-\mathrm{H}]^{-}$not observed } \\
\hline Small rock & IMPA & YES & {$[\mathrm{M}-\mathrm{H}]^{-}$not observed } \\
\hline Small rock & EMPA & YES & {$[\mathrm{M}-\mathrm{H}]^{-}$not observed } \\
\hline Small rock & MPA & YES & {$[\mathrm{M}-\mathrm{H}]^{-}$not observed } \\
\hline Glass & IMPA & YES & weak [M-H]- observed \\
\hline Teflon tape & IMPA & NO & analyte evaporates \\
\hline Floor Tile & MPA & YES & [M-H]" observed \\
\hline Ceiling Tile & MPA & NO & $63^{-}, 79^{-}, 95^{-}$not observed \\
\hline
\end{tabular}




\section{Instrument Development}

\section{Ion Trap Molecular Beam Surface Analysis Instrument}

This section describes the design and current prototype of the MBSA instrument configured with an ion trap mass spectrometer and the details of the primary components. While the system is laboratory based, it is being designed to demonstrate the potential for producing a field-transportable version. The instrument design is driven by several goals, all constrained by the desire to keep the system highly flexible, robust, and well suited for field operation by non-technical personnel. In addition, it is required that the system have a very low potential for producing false-positives. The design goals include: 1) obtaining high selectivity, 2) high sensitivity, 3) the ability to accept a wide range of sample types, and 4) simplicity in operation. All of these are subject to compromise such that the overall design provides the best result for the end-user, and it is recognized that different end users may have slightly different sets of priorities, thus the need to keep the design flexible at this stage of development. It is intended that the prototype instrument will clearly demonstrate the overall potential of the technique, and make it clear what tradeoffs would be necessary to enhance any single performance goal.

\section{Instrument Design}

The conceptual design of the MBSA instrument is shown in Figure 1. The system includes an ion trap mass spectrometer, operated from a personal computer, and contained in a small vacuum housing equipped with a sample introduction probe. When inserted into the vacuum chamber, the probe holds the sample where it can be bombarded by the primary ion beam such that the secondary ions sputtered from the sample are efficiently transported into the ion trap. The window in the lower right of Figure 2 illustrates the operation of the instrument in a standard acquisition mode. The primary beam is first pulsed onto the sample for a short duration and secondary ions of positive polarity are collected in the ion trap. During this period the beam deflector grids, multiplier, and dynode are at zero voltage, allowing the primary beam to

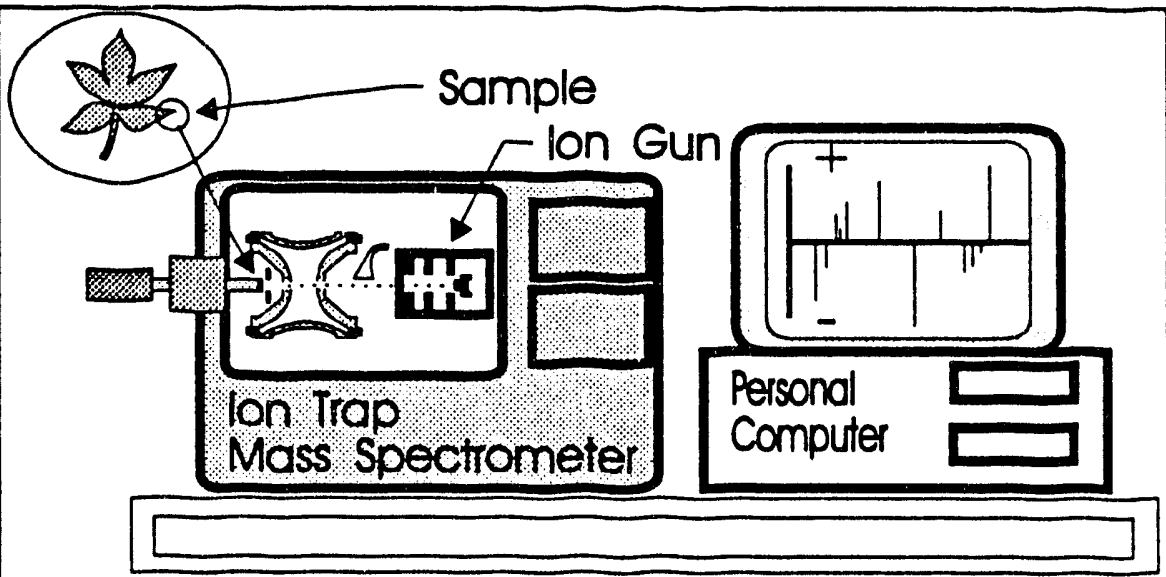

Figure 1. Conceptual design of the ion trap based MBSA benchtop instrument. pass through the detector region and the ion trap and strike the sample; the secondary ion lens is set to the appropriate voltage and polarity to direct ions into the ion trap; and the ion trap RF and DC voltages are set to optimize trapping of the ions. Following the ion generation pulse the ions are held in the ion trap briefly for collisional cooling, and then they are ejected out of the trap in a mass dependent sweep to the detector. During this period the deflector grids and dynode are set at voltages that direct the cations onto the detector. The positive ion mass spectrum is collected and stored in the data system. The cycle can then be repeated in an identical manner, or repeated with the secondary ion lens polarity reversed to collect the anions, and keep the sample from building up charge if it is a non-conductor. Note when detecting anions 
the dynode voltage is set to attract the anions for conversion to cations prior to striking the detector.

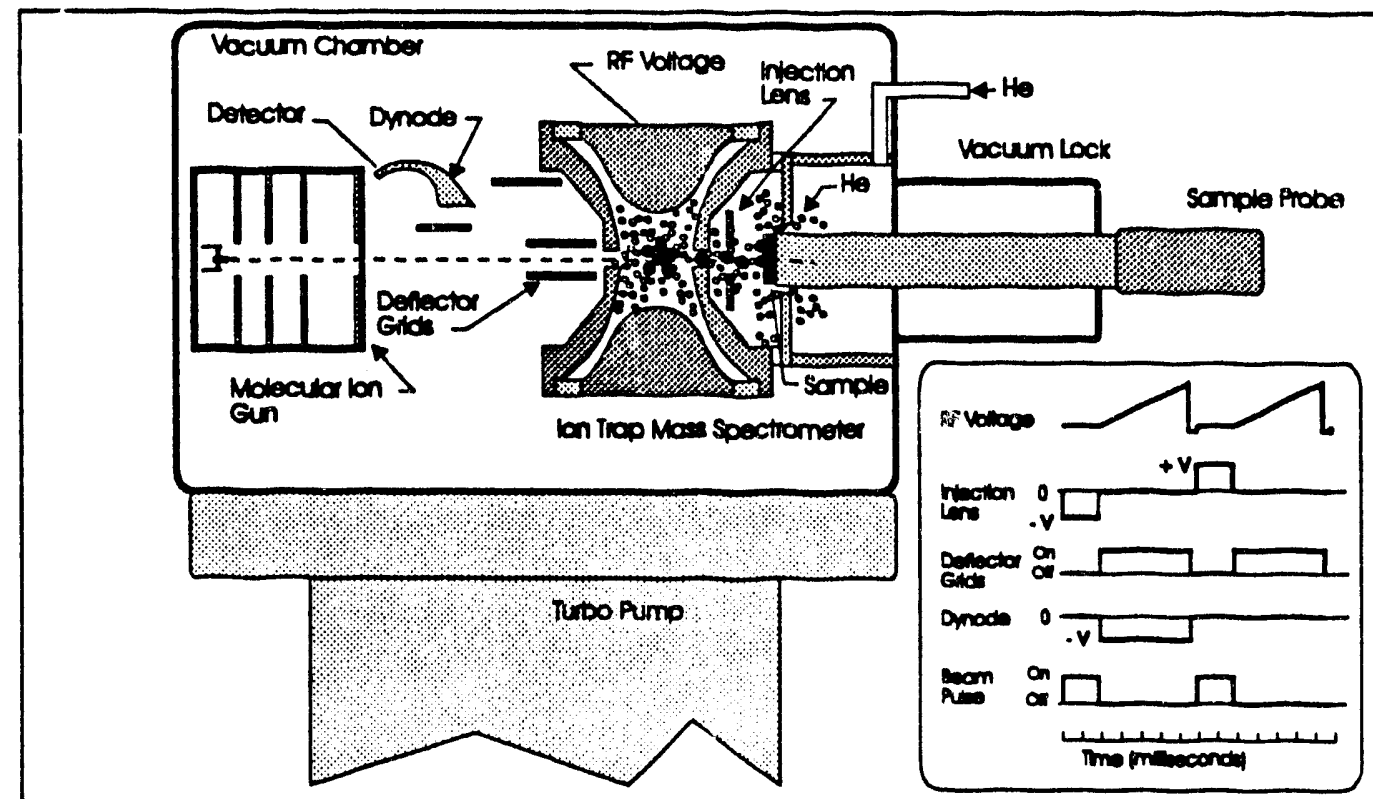

Figure 2. Conceptual design of the electrostatic and collisional focusing of secondary ions into the ion trap and a generalized operational sequence for pulsed extraction operation of the system.

Depending on the particular goal of the analysis, this generalized sequence can be modified to perform a wide range of different routines, including strategies such as collecting ions from multiple pulses,

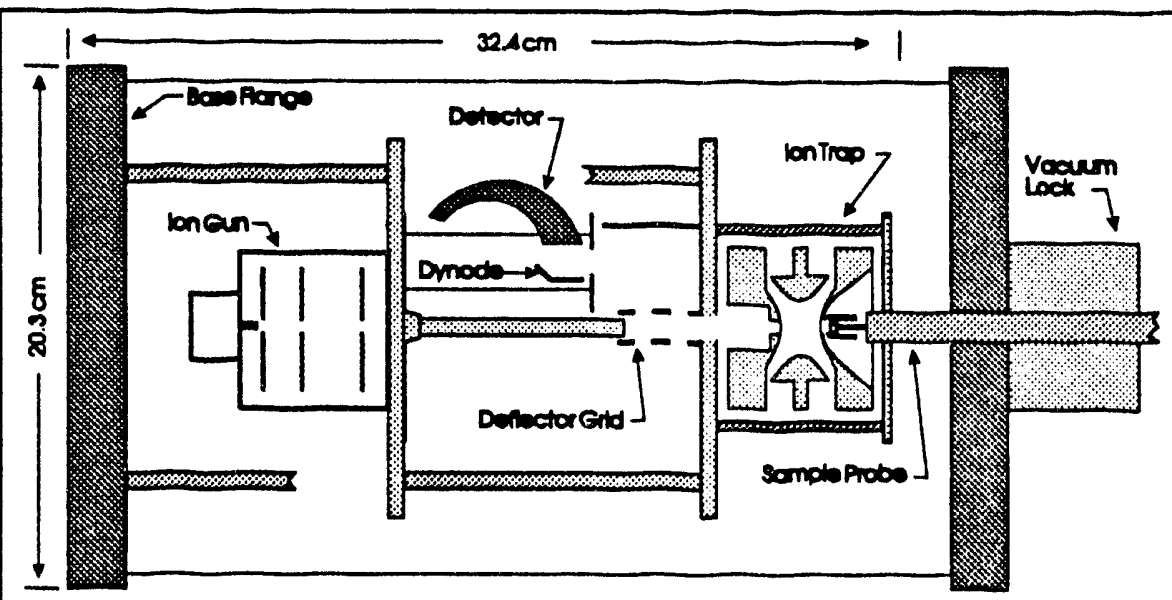

Figure 3. Scaled drawing of the current prototype MBSA instrument. collecting ions of only a particular mass, performing MS/MS on a particular mass ion, etc. This flexibility is a major feature of the ion trap based system that makes it robust and able to adapt to a wide range of requirements. A scaled drawing of the current MBSA prototype is shown in Figure 3. However, rather than discuss the design of the system on a component by component basis, in the following we describe the design relative to the overall system goals listed above so that the role the components play in meeting these goals is more readily apparent.

Selectivity The primary component controlling the selectivity and specificity is the mass spectrometer. Both the molecular ion and fragment ions of $\mathrm{CW}$ decomposition products are typically observed in the 
secondary ion mass spectra and the observation of these ions at abundances above the background gives a high level of confidence that the decomposition products can be unequivocally identified. It is anticipated, however, that the spectra of some samples will contain considerable chemical noise, which can confuse the interpretation of the data. In this case, it will be necessary to either collect bigh resolution spectra or perform mass spectrometry/mass spectrometry (MS/MS) in order to decrease the chance of faise positive detections from isobaric ions arising from the background. This was one of the primary motivations for utilizing the ion trap mass spectrometer, along with its compact size. Ion traps can perform MS/MS analyses without any additional hardware, and can even perform higher order analyses $\left(M^{n}\right)$ if needed. In addition, new operating modes are now making it possible to perform high resolution scans, which can augment the MS/MS function. The ability to perform MS/MS on a sample is expected to make it possible to determine that a mass peak does correspond to the target chemical with very high certainty. A more detailed discussion of the general MS/MS capabilities of the ion trap mass spectrometer is given in Appendix 1-.4. We have performed MS/MS analyses on PMPA to illustrate and test this

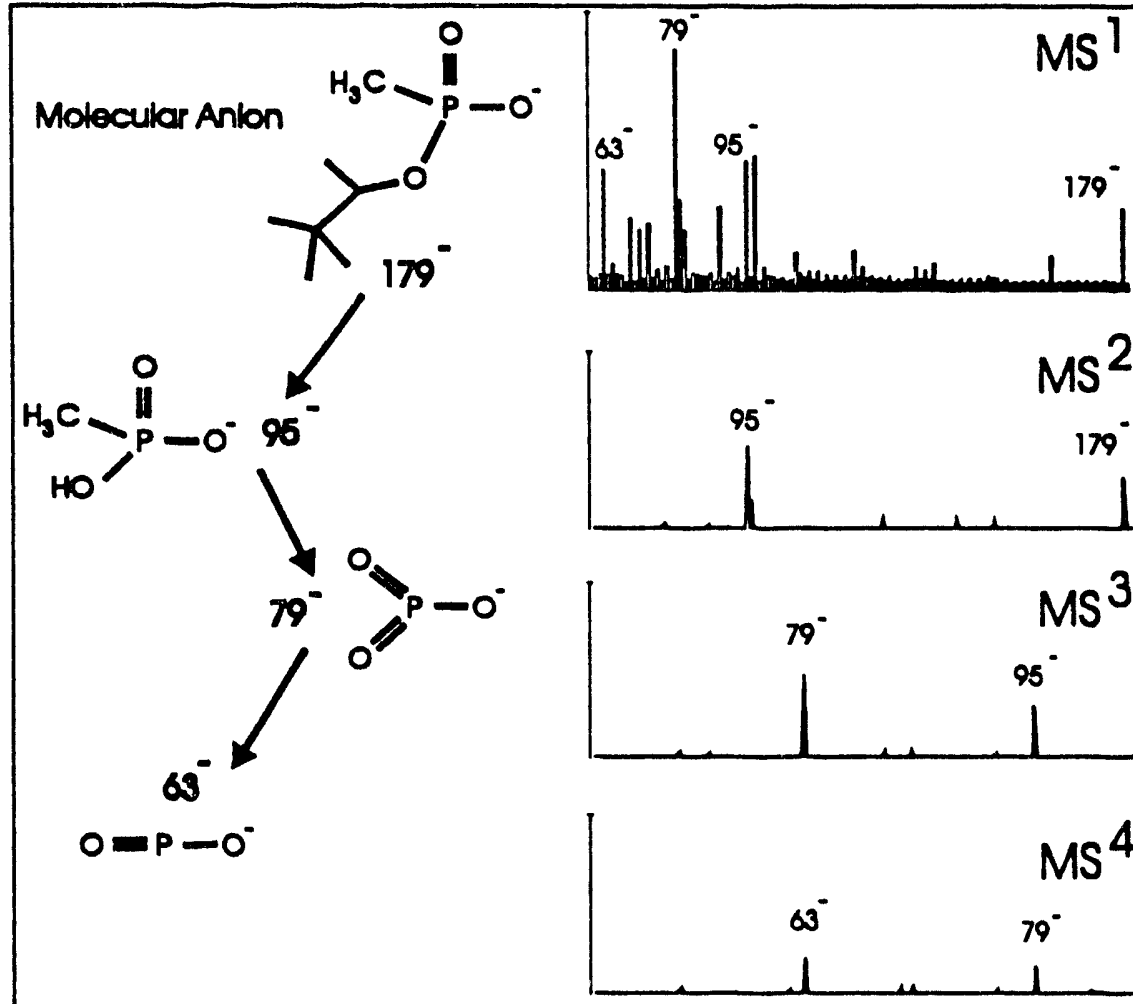

Figure 4. Four stages of mass spectrometry with collision induced dissociation at each step. PMPA on stainless steel substrate.

confidence, this does illustrate the power and efficiency of the ion trap system. The exploration of MS/MS on the MBSA has been only cursory up to this point in the system development, more detailed studies are scheduled for the upcoming year, and yet these limited results have confirmed our assertion that this will provide the needed level of specificity. The primary challenge will be developing a data system with which MS/MS analyses can le easily performed (an area that several of the commercial ion trap mass spectrometer vendors are currently addressing).

It is also clear that the system must be able to detect both positive secondary ions and negative secondary ions. The most analytically significant peaks for the AMPAs are in the negative ion spectrum, while for the mustards they are in the positive ion spectrum. The system currently can analyze either polarity ion, and it is possible, with the appropriate additions to the data acquisition system, to collect both polarity spectra simultaneously. The design of the detector system includes the capability to perform simultaneous detection, as illustrated in Figure 5. Since the ion trap will eject both positive and negative ions at the 
same time, they can be separated and detected independently. Our experience with a quadrupole mass spectrometer system that collects both the positive and negative ions, the system used to perform the a nalytical studies, has shown this to be a significant time saver, particularly when looking at unknown samples. We plan to explore this capability for the ion trap system in the upcoming year. The system currently operating in the ion trap instrument includes only a single detector, and a conversion dynode is employed for detecting negative ions.

Sensitivity The sensitivity will be dependent on the efficiency with which secondary ions are produced,

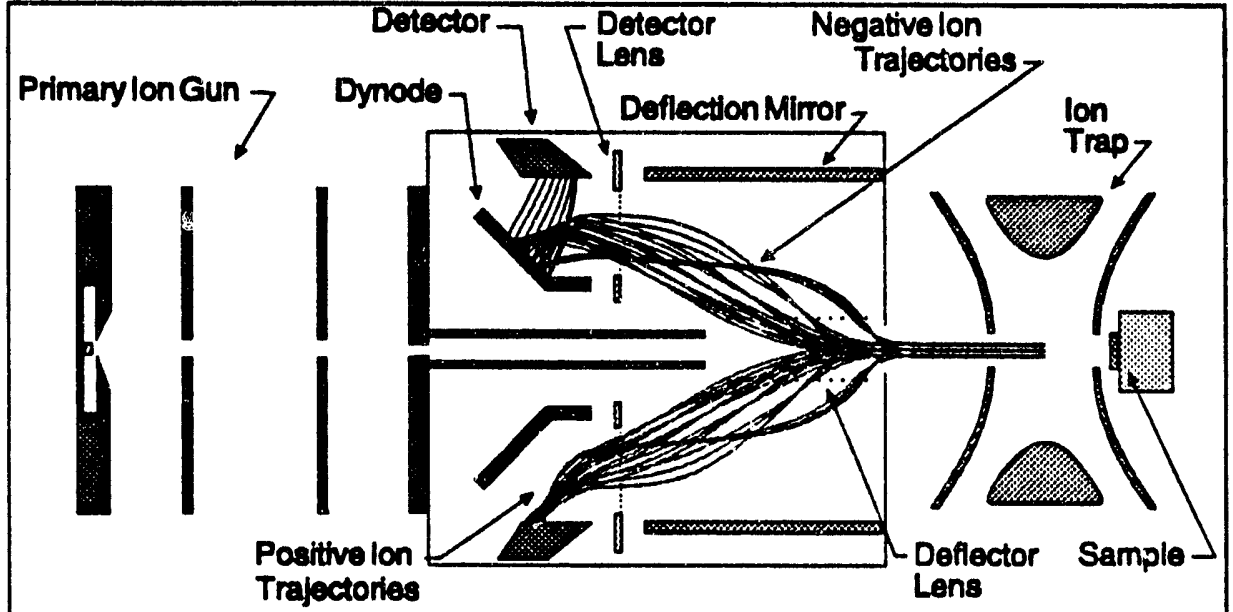

Figure 5. SIMION ${ }^{1}$ calculated ion trajectories for a dual detector system. Ion energies range from $5 \mathrm{eV}$ to $500 \mathrm{eV}$. The current ion trap MBSA instrument utilizes only a single detector in this geometry. the efficiency of transporting the ions into the ion trap and trapping them, and the efficiency of ejecting them from the trap and their subsequent detection. The first step is producing the secondary ions from the sample. We have shown ${ }^{2}$ in previous work that a molecular primary beam is 10 to 30 times more efficient at producing secondary ions

from the types of samples of interest to this program than standard atomic ion beams commonly used in SIMS. The ceramic molecular ion source used in the system is incorporated into a simplified ion optics train designed for high stability. The ion beam is shot directly through the ion trap, making it possible to keep the sample very close to the ion trap end cap, and minimizing the size of the vacuum chamber. Tests conducted on one of our research instruments has shown that the loss in primary ion beam intensity suffered by passing the primary beam through the ion trap filled with He will be only on the order of $10 \%$, a very good compromise given the advantages of the collinear design. In the design of the sample probe and secondary ion collection optics, as illustrated condeptually in Figure 2, the first goal was to keep the sample as close to the ion trap entrance as possible and use the minimum number of ion optical components. Our current design utilizes two mechanisms for moving the secondary ions sputtered from the sample into the ion trap: collisional focusing and electrostatic focusing. We are conducting experiments to define the best combination of these two mechanisms. Since the ion trap has historically operated optimally with an over pressure of $\mathrm{He}$ gas in the trap, one option is to use the helium gas flow to belp guide the secondary ions into the trap, as illustrated in Figure 2. In this configuration the primary conductance path for the helium to enter the trap will be around the sample, thus the He molecules will collide with the secondary ions as they are sputtered from the sample. This will do two things; it will tend to move the secondary ions in the general direction of helium flow, into the trap, and it will tend to damp

'Dahl, D.A.; Delmore, J. E.; SIMION PC/PS2 User's Manual, EG\&G-CS-7233, 1988.

${ }^{2}$ Appelhans, A. D.; Delmore, J. E.; Anal. Chem.; 1989; 61; 1087-1093. 
the secondary ion kinetic energy, moving all secondary ions toward a low, average kinetic energy. The injection lens serves two purposes, to focus the secondary ions into the trap aperture, and to control the polarity of the ions focused into the trap. In order to take advantage of pulsed extraction sample neutralization, the polarity of the ions extracted from the sample must be alternated between positive and negative; this is controlled with the electrostatic lens element. Figure 6 shows a SIMION model of the secondary ion extraction lens and the predicted trajectories of ions ejected from the sample with no helium present (collision free field). It's clear that the system provides a high efficiency transfer of the ions into the trap. However, if there is a high pressure of helium in this region resulting in multiple collisions, the ion trajectories are going to be significantly altered, possibly reducing the transfer efficiency. Thus it may be best to have the helium buffer gas inserted directly into the ion trap, as opposed to the sample region. While this can be modeled statistically with SIMION, we plan to test each configuration on the instrument

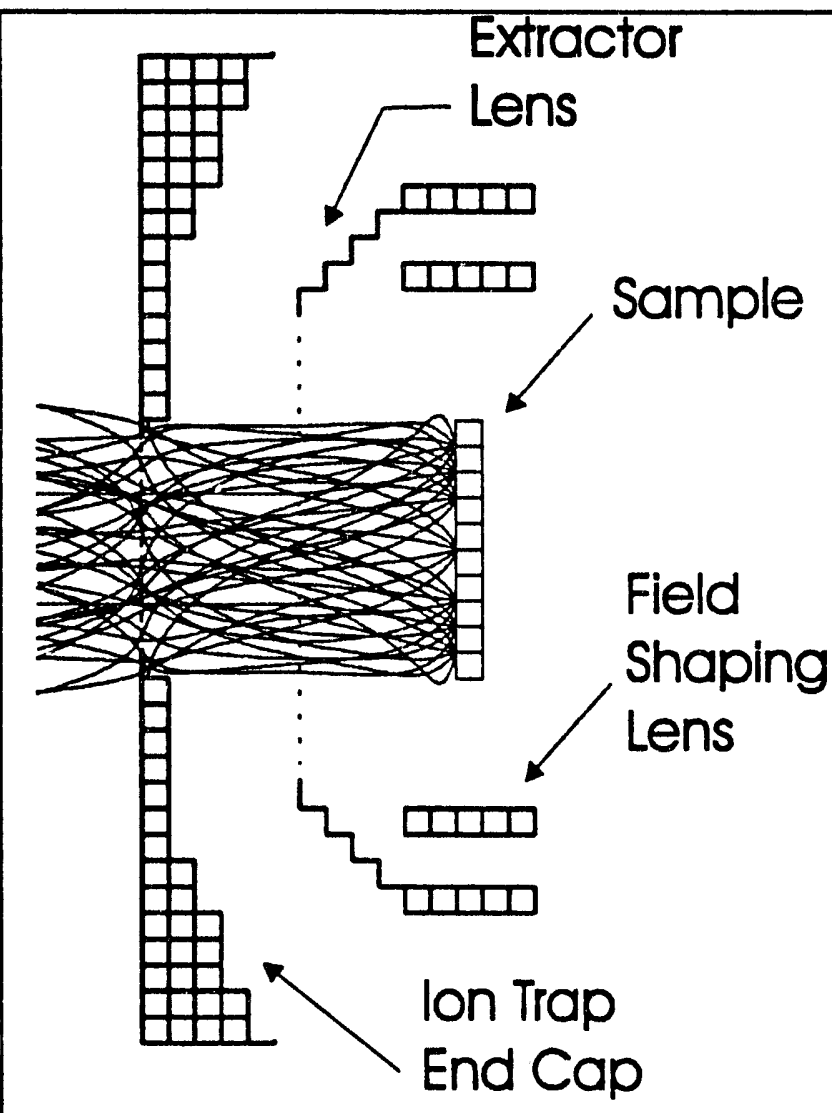

Figure 6. SIMION calculated secondary ion trajectories for the sample inlet region of the ion trap, assuming no col'sions with the helium buffer gas. experience of others ${ }^{4}$ indicates an increase of an order of magnitude in sensitivity using this technique.

${ }^{3}$ HST-1000, Teledyne MEC, Mountain View CA

${ }^{4}$ Goeringer, D. E.; Asano, K. G.; McLuckey, S. A.; Stiller, S. W.; Hoekman, D; $M S^{n}$ in the Quadrupole ion Trap Using Filtered Noise Fields, 41st ASMS Conference, San Francisco CA, June 1993.
The efficiency of trapping the ions will be optimized by controlling the RF and DC voltages applied to the trap electrodes during ion insertion, and controlling the helium pressure in the trap. Our recent experience has shown that the ion trapping efficiency is quite sensitive to the helium pressure. The pulsed operation of the ion trap permits a wide range of options for trapping ions, and it is anticipated that several strategies will evolve for optimizing the trapping efficiency. In order to obtain the highest sensitivity possible it is also desirable to fill the ion trap only with ions that are of analytical interest. Particularly in real-world samples there are many ions that are of little analytical interest, but that are produced in high abundance. Their presence in the ion trap limits the number of analytically significant ions that can be in the trap, since space charge effects place a limit on the total number of ions that can be in the trap. Methods have been developed ${ }^{3,4}$ that utilize filtered noise fields that make it possible to collect only ions within certain ranges, and to exclude ions outside of these ranges or in other specific ranges, making it possible to collect only ions that are analytically useful. We have initiated incorporation of this technology in the MBSA ion trap instrument, but at this time have no results to report. The 
The final consideration with respect to sensitivity is the efficiency of ejecting the ions from the trap and detecting them. The major parameters affecting this will be the ion trap operating parameters, the scan out function (of which there are a wide variety of options to explore), and the efficiency of the detection system. It has been shown ${ }^{5}$ that the trap can be operated to selectively control the polarity of the ions ejected out of one or the other side of the trap, making it possible, for example, to choose to eject only the positive ions into the detection system. In a normally operated ion trap half the ions are ejected out one side, the other half out of the other side of the trap, inherently reducing sensitivity. Using this new method the sensitiv ty could be increased by approximately a factor of 2 , while giving up the option of simultaneously collecting both polarity ions. The decision on which of these options to use will be based on the end-user needs. With respect to the detection system, the current system utilizes a conversion dynode for negative ions, and detects positive ions directly, using a channeltron multiplier. The SIMION model of this system (Figure 5) indicates it has a bigh acceptance efficiency, handling ions ranging from a few eV up to $500 \mathrm{eV}$ of kinetic energy, and our experimental results confirm this. Since the energy of the ions ejected from the ion trap is proportional to their mass, this broad energy bandwidth is particularly important for detection of higher mass ions. Further testing of the ion detection system geometry is planned for the upcoming year.

Sample Preparation and Insertion The ease with which a sample is prepared and introduced into the system will have a direct bearing on the cost per analysis, both with respect to time and money, and it can affect the quality of the data. The current sample probe has been designed to accommodate a wide variety of sample types, ranging from plant leaves to pieces of gravel, such that all that is required of the operator is to cut the sample to the appropriate size $(\sim 0.5 \mathrm{~cm}$ diameter), attach it to the probe tip (double stick tape is currently used, a variety of quick-connect probe tips may be utilized to accommodate different sample geometries), and insert it into the vacuum system. There is no sample preparation required other than cutting to size. The current system utilizing a double sided tape to attach the sample to the probe tip has worked very well for a wide variety of samples, making it possible to mount a typical sample in less than a minute. The insertion vacuum lock is a dual chamber design that permits the sample to be desiccated in the lock if necessary, prior to insertion into the main vacuum chamber. The pumping requirement for the insertion lock will be dependent on the type of samples the end-user anticipates handling, and thus would have to be determined based on the end-user needs. On our current laboratory based system the insertion lock utilizes the roughing pump of the main chamber turbo pump.

Operational Simplicity It is a goal to design the system so that there are very few adjustments required for operation, with as few parts as possible, to demonstrate the potential for building a system that could be operated in the field by a technician. Thus the designs of all of the components and subsystems will be evaluated to determine the optimum tradeoff between simplicity performance. The operation of the primary beam gun using the ceramic ion source is an example. This ion source has been in operation in the ion trap system for $\sim$ six months with no servicing required. The ion gun can be brought up from cold shutdown to operational status in $\sim 3$ minutes, and all power supplies associated with the system are in a single $12 \mathrm{~cm}$ high rack mount unit. The ion lens is a simple two-element system with a total length of only $\sim 3 \mathrm{~cm}$. The general focusing characteristics have been confirmed during testing of the ion gun in a separate ion beam test stand equipped with a multichannel plate image intensifier for viewing the beam profile. In contrast to the simplicity of the ion gun system, the current data acquisition system (Finnigan ITMS) is designed for operation by researchers, requiring a relatively high level of skill, training and understanding to program and operate. Thus a major task that will remain before this type of system is truly ready for operation in the field by a technician will be to provide an easy-to-use data acquisition system. Fortunately the recent introduction of three new vendors in the ion trap field bas spurred on development, and we expect to see several new options available in future systems that will make them much easier to use.

${ }^{5}$ Marquette, E.; Wang, M.; Uni-Directional ion Ejection in Ion Trap, 41st ASMS Conference, San Francisco CA, June 1993. 


\section{Direction of Current Work}

Efforts in the upcoming year will focus on optimizing and refining the design and operational protocols of the instrument. Starting with the ion trap itself, we are planning further studies to optimize the sizing of the beam apertures in the end caps. Currently these apertures are covered with a high transmission grid (85\% open area) in order to maintain the shape of the electric field inside the ion trap. Optimization will include varying the aperture size and the grid spacing, with the goal of obtaining the largest practical aperture size and grid spacing. This is important because aperture size defines the area of the sample that can be analyzed. In addition to physical optimization of the ion trap, the operational modes must be investigated and optimized. A filtered noise system is being incorporated into the system, and this will be tested in a variety of modes to help define the optimum operational conditions. With respect to MS/MS analysis, the possible advantages of pulsing in a larger mass collision gas is under consideration.

Optimizing the efficiency for getting the ions into the trap is an area that will receive much attention. Variations on the extraction lens design will be explored along with testing of the best position for introduction of the helium buffer gas. One option being considered is pulsing the helium in concert with the extraction lens to provide a high density of gas during the trapping period, followed by a lower density during mass separation and detection. The incorporation of pulsed secondary ion extraction into the operating mode of the ion trap system will be tested and evaluated.

Detection of the ions will also be optimized. The importance of high detection efficiency for negative ions is particularly important since most of the analyticaily useful information resides in the negative ion spectra for the major CW degradation products (see later sections of this report). We believe we can significantly improve the detection efficiency of the current system, both using new modeling tools soon to be available (SIMION 6.0) to optimize the system geometry, and by improving the "conversion" efficiency of the dynode. The potential advantages of incorporating a dual detector system will be carefully evaluated prior to full implementation because of the major modifications to the data system that such an option would require.

The present prototype instrument is providing us with a baseline upon which to measure the pros and cons of these planned modifications. We are very pleased with the current performance of the overall system, but also feel that we can make significant gains. All of our experience up to now indicates we should be able to meet our goal of clearly demonstrating and defining the performance of an ion trap molecular beam surface analysis instrument constructed with components that can be easily engineered for field applications. 


\section{Sensitivity Study}

\section{Minimum Detectable Levels of Alkylmethylphosphonic Acids on Plant Leaves}

\section{Summary}

Minimum detection levels were measured for chemical weapons degradation products deposited on plant leaves to provide a benchmark for evaluating the performance of the ion trap mass spectrometer based molecular beam surface analysis instrument currently under development. A second objective in this study was to evaluate factors governing the detection limits. These measurements were performed using a quadrupole secondary ion mass spectrometer. The analytes used in this investigation were the primary degradation products of VX, GB, and GD, generally classified as alkylmethylphosphonic acids (AMPAs). The lower limit of detection was approximately $4 \mathrm{ng} / \mathrm{mm}^{2}$ ( 4 monolayers) for determining that an AMPA was present on a sample, and was a factor of ten higher for determining the specific agent degradation product. In both cases, the measurements were limited primarily by chemical noise (all of the background chemicals typically present on environmental samples). This corroborates our prediction that in order to obtain the highest sensitivity and specificity, a mass spectrometer capable of performing mass spectrometry/mass spectrometry is required. The ion trap mass spectrometer system currently under development has this capability. The detection limits determined in this study are consistent with our previous scoping studies on a wider variety of plant species, indicating the limits are not strongly dependent on the type of plant chosen for the sample.

\section{Introduction}

This section summarizes the results of an evaluation of the detection limits of quadrupole mass spectrometer based molecular beam surface analysis (MBSA) for chemical weapon (CW) degradation products on plant leaves. The purpose of this study was to ascertain and evaluate factors governing detection limits related to MBSA. Since the ion trap based MBSA instrument is still under development and was not available for this study, an existing quadrupole based MBSA instrument was employed. These measurement limits are believed to be conservative estimates of the lower limit of detection, as it is expected that the ion trap based instrument will have higher sensitivity.

A major question to be addressed is the ability to distinguish not just that an agent bad been present on a sample, but to be able to specifically identify which agent was present. In these studies, lower levels of detection were determined for both cases. The agent group that presents the most difficult challenge for specificity are the nerve agents composed of VX, GB, and GD. The degradation of each of these agents in the environment leads to a class of chemicals termed alkylmethylphosphonic acids (AMPAs). Thus, the presence of this class of compounds on a sample is an indication that one of the agents had previously been present. In order to determine which specific compound had been present requires detecting the complete molecular ion. Thus, this group of compounds is an excellent test for both sensitivity and specificity of the detection method.

\section{Experimental}

The MBSA was performed using a quadrupole based secondary ion mass spectrometer (SIMS) developed in this laboratory. It is equipped with two unique features: a) perrhenate $\left(\mathrm{ReO}_{4}{ }^{-}\right)$primary ion beam and b) pulsed secondary ion extraction for sample-charge neutralization. The perrhenate ion gun produces a large molecular anion that is highly efficient for sputtering molecular ions from a sample surface, giving improved sensitivity over conventional atomic ion guns. These attributes result in the analysis of only the 
top few monolayers of sample. Pulsed extraction alternately samples positive, then negative ions. By varying the positive/negative ratio, sample surface charging can be minimized. This allows analysis of insulating samples such as leaves, which are not readily analyzed using conventional SIMS. A more detailed description of the instrument may be found in the listed references. 1,2

\section{Sample Preparation}

The chemicals used in these experiments pinacolyl methylphosphonic acid (PMPA), isopropyl methylphosphonic acid (IMPA), and ethyl methylphosphonic acid (EMPA) are listed in Table 1 along with the corresponding CW agent from which they degrade. The chemicals were obtained from CRDEC, Aberdeen in April 1992 in the form of 100 pp. aqueous solutions. These solutions were used as received. No analysis was performed in our laboratory to verify the concentrations. These solutions were diluted by a factor of $\mathbf{1 0}$ and $\mathbf{1 0 0}$ with nanopure water in order to investigate three orders of magnitude difference in surface loading.

Table 1. Agents and degradation products

\begin{tabular}{|c|c|c|c|}
\hline Agent & Primary degradation product & Structure & Acronym \\
\hline vX & Ethyl methylphosphonic acid & & EMPA \\
\hline GB (sarin) & Isopropyl methylphosphonic acid & & IMPA \\
\hline GD (soman) & Pinacolyl methylphosphonic acid & & PMPA \\
\hline
\end{tabular}

The leaves used in these experiments were taken from a common house plant, PHILODENDRUM scandens oxycardium. Fresh leaves were used for analysis each day of the experiment. Disks of $3.8 \mathrm{~mm}$ diameter were cut from the leaf with a bole boring tool. The geometric sampling area was $11.34 \mathrm{~mm}^{2}$. The leaf disks were attached to a stainless steel planchette using double-stick tape. After attaching the leaf disks to the planchettes, the leaf disks were spiked with $5 \mu$ of the solution of interest. The spiked leaf was allowed to air dry for 45 minutes before analysis. The sample was then inserted into the instrument and analyzed at a pressure of $1 \times 10^{-6}$ torr.

The calculated mass per unit area of these loadings, assuming the original concentration of the received solutions at $100 \mathrm{ppm}$, were: $44 \mathrm{ng} / \mathrm{mm}^{2}(100 \mathrm{ppm}), 4.4 \mathrm{ng} / \mathrm{mm}^{2}(10 \mathrm{ppm})$, and $0.44 \mathrm{ng} / \mathrm{mm}^{2}(1 \mathrm{ppm})$.

${ }^{1}$ Appelhans, A. D.; Dahl, D. A.; Delmore, J. E. Anal. Chem., 1990, 62, 1679-1686.

2Delmore, J. E.; Appelhans, A. D. Biol. Mass Spec., 1991, 20, 237-246. 
Note that evaporation, absorption, or other losses of the spiked material were not accounted for in these calculations. Also, the sampling area was calculated strictly from the dimensions of the disk; porosity, surface roughness, and other parameters effecting the surface area were not considered in the calculation. Thus, the calculated loading values were most likely larger than the quantity of material actually analyzed. In order to calculate surface coverage, it was assumed that the AMPAs would deposit in a upright manner with respect to the surface. This assumption is very const.vative as it implies the highest number of molecules per unit area. Assuming each molecule to bave an area of $20 \AA^{2}$, based on the same orientation of a fatty acid ${ }^{3}$, the following surface coverages were estimated: $100 \mathrm{ppm}-40$ monolayers (ML), $10 \mathrm{ppm}-4 \mathrm{ML}$, and $1 \mathrm{ppm}-0.4 \mathrm{ML}$.

These three levels of surface coverage were chosen based on our previous scoping studies. 1 We expected to easily detect the highest loading and felt that the lowest loading would be below our limit of detection.

\section{Data Acquisition}

Three scans over the mass range of interest were signal averaged for samples spiked at $40 \mathrm{ML}$, total analysis time was ca. 8 minutes per sample. Five scans were signal averaged for samples spiked at $4 \mathrm{ML}$, total ana iysis time was ca. 15 minutes per sample. Ten scans were signal averaged for samples spiked at $0.4 \mathrm{ML}$, total analysis time was ca. 30 minutes per sample. Eight sets of data were collected at each loading level, using new leaves each time. These data were collected on eight separate days. An optimization procedure for sample placement and minimization of sample charging was employed before each sample was analyzed to ensure sample-to-sample consistency in analysis. Blanks consisting of both the bare leaf and leaves spiked with water only were also analyzed in a similar fashion to determine the background.

Only the anion spectra are presented in this report; the cation spectra did not contain information useful in identifying the species of interest. The spectra were normalized to the $25^{-}$peak $\left(\mathrm{C}_{2} \mathrm{H}^{-}\right)$to account for minor fluctuations in primary beam intensity from day to day. This peak was chosen as it was reproducibly present on both the leaf blank and the AMPA spiked leaves. The "mean relative abundance" label on the $y$-axis of the spectra in the following figures represents the average of the 8 sets of data collected for each analyte normalized to the $25^{\circ}$ peak.

\section{Results}

\section{Spectral Interpretation}

Background spectra were acquired for both a bare leaf and a leaf spiked with water only. In Figure 1, an averaged negative ion spectrum of a blank leaf is shown; this spectrum is an average of 8 sets of data taken on 8 different days. The spectrum exhibits a strong peak at $25^{-}$which is attributed to $\mathrm{C}_{2} \mathrm{H}^{-}$and is typically observed from organic surfaces. The peak at $41^{-}$is attributed to $\mathrm{HC} \equiv \mathrm{C}-\mathrm{O}^{-}$which arises from the waxy alcohols that make up much of the epicuticular waxes of plant leaves ${ }^{4}$. This peak corroborates the hypothesis that the plant leaves used in these studies are hydrophilic in character. The peak at $42^{\circ}$ is believed to be due to $\mathrm{O}=\mathrm{C}=\mathrm{N}^{-}$. Confirmation of this assignment arises from the presence of a peak at $26^{-}$ which corresponds to $C \equiv N^{-}$. In Figure 1 the ratio of 25:26 is much larger than in Figure 2; correspondingly, the 41:42 ratio is larger in Figure 2 than in Figure 1. Reported analyses of the epicuticular waxes do not indicate the presence of nitrogen-containing compounds, such as amines or

${ }^{3}$ Adamson, A. W. Physical Chemistry of Surfaces, John Wiley \& Sons, Inc., New York, 1990, pg. 435.

${ }^{4}$ Baker, E. A.; Stevens, P. J. G.; Pestic. Sci.; 1987; 19; 265-281. 


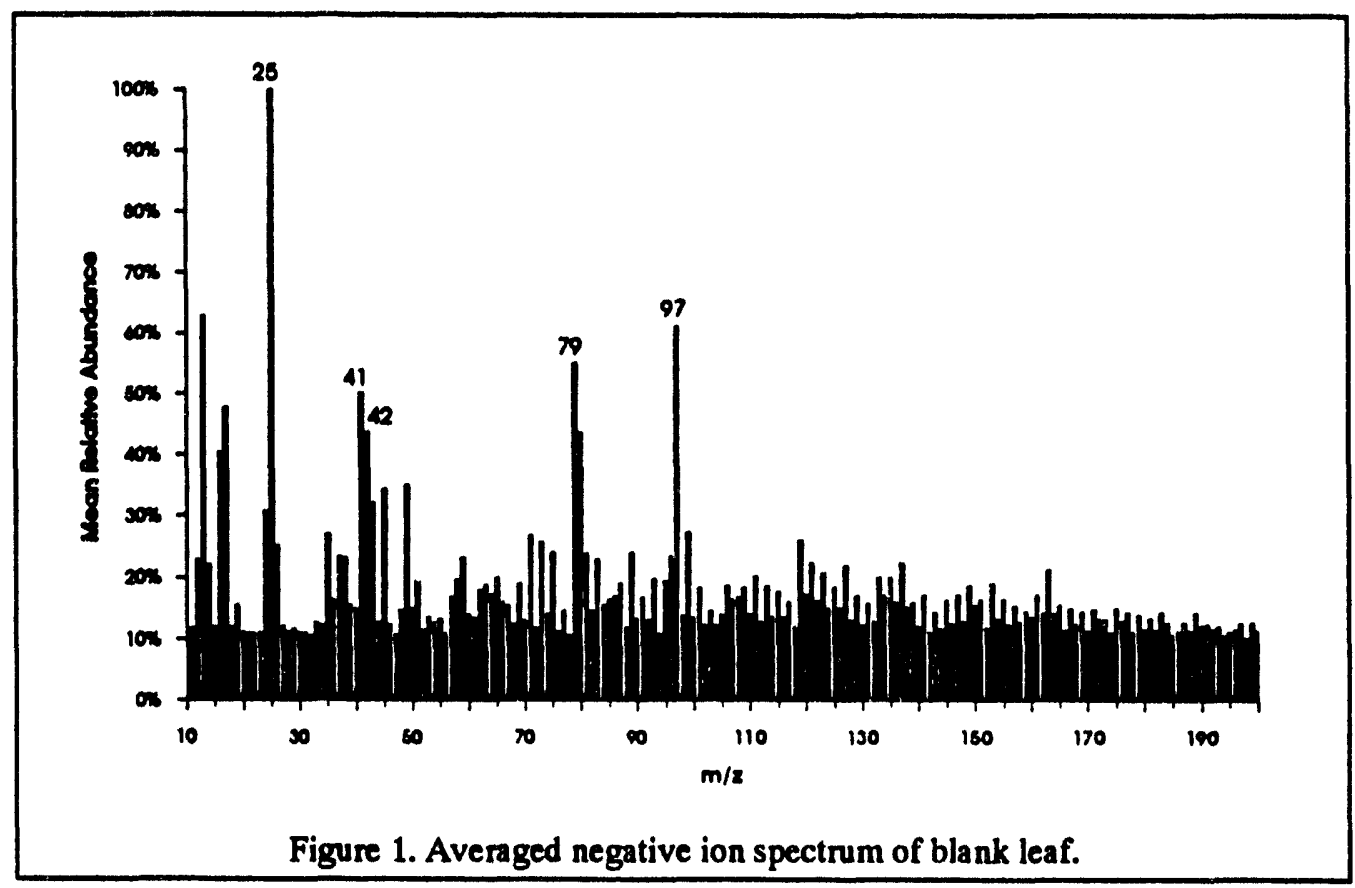

amides ${ }^{4,5}$. These results strongly suggest the presence of a nitrogen-containing compound on the leaf surface. Additionally, SIMS analysis of a variety of other plant leaves also exhibit the $26^{-}$and $42^{-}$peaks (see section IV of this report). Further investigations are underway to identify the origins of these peaks.

The peak observed at $79^{-}$is attributed to $\mathrm{PO}_{3}{ }^{-}$which originates from phosphate and is somewhat

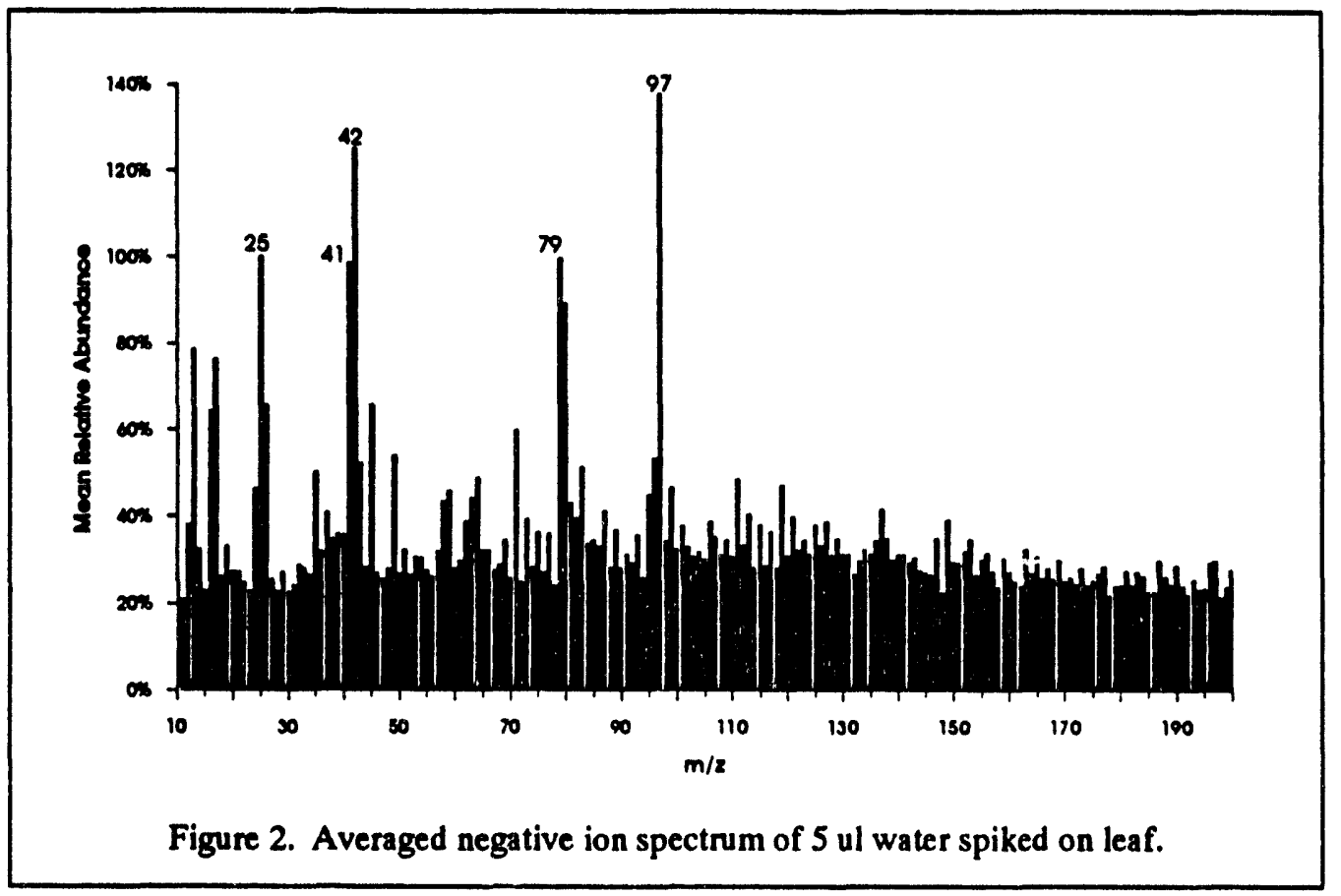

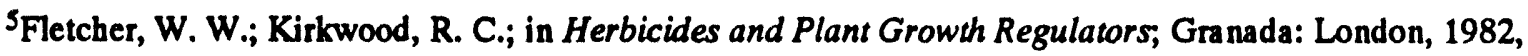
p104. 
ubiquitous in the environment as a result of detergents, pesticides, and other phosphate-containing

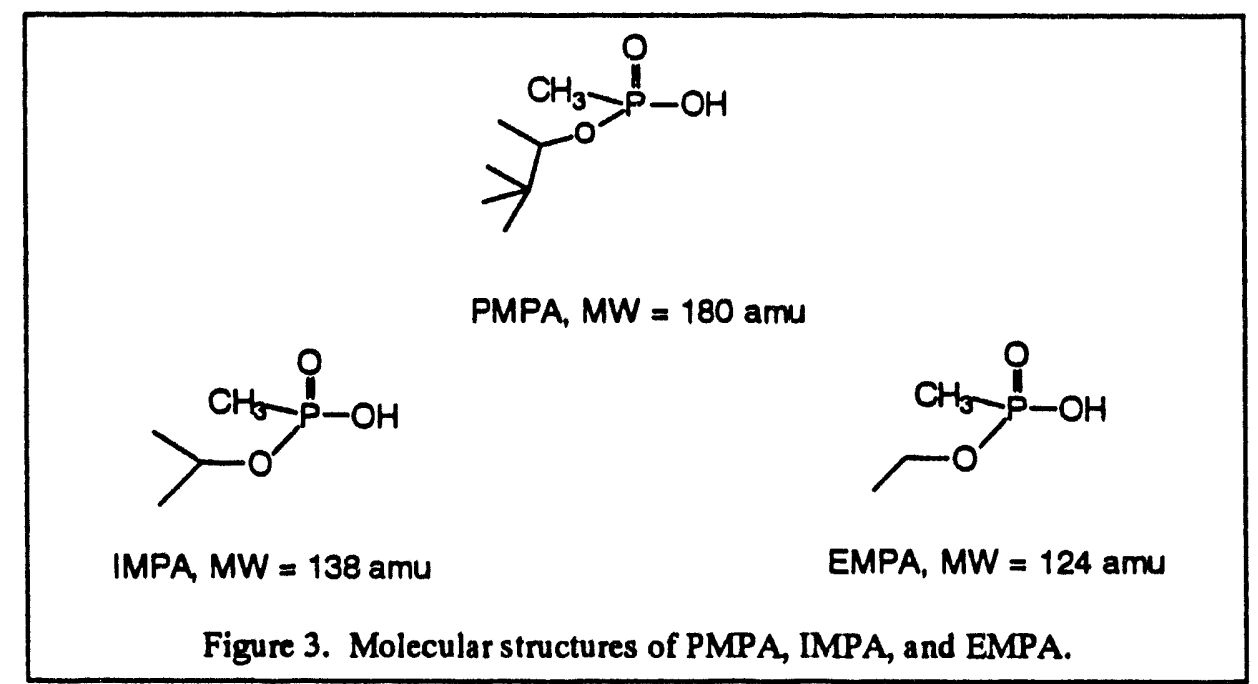

materials. The peaks at $80^{-}$(unmarked) and $97^{-}$are attributed to $\mathrm{SO}_{3}^{-}$and $\mathrm{HSO}_{4}^{-}$, respectively, and a re also quite prevalent in the environment. In addition to these major peaks, a significant "background" signal is observed at every mass. This background is primarily a result of chemical noise, that is, it arises from the wide variety of atomic and molecular ions sputtered from the sample, and also produced in the quadrupole mass spectrometer by high energy collisions. The electronic noise in the detection system is two orders of magnitude below this chemical noise level.

Leaves spiked with nanopure water only were analyzed to determine background peaks due to

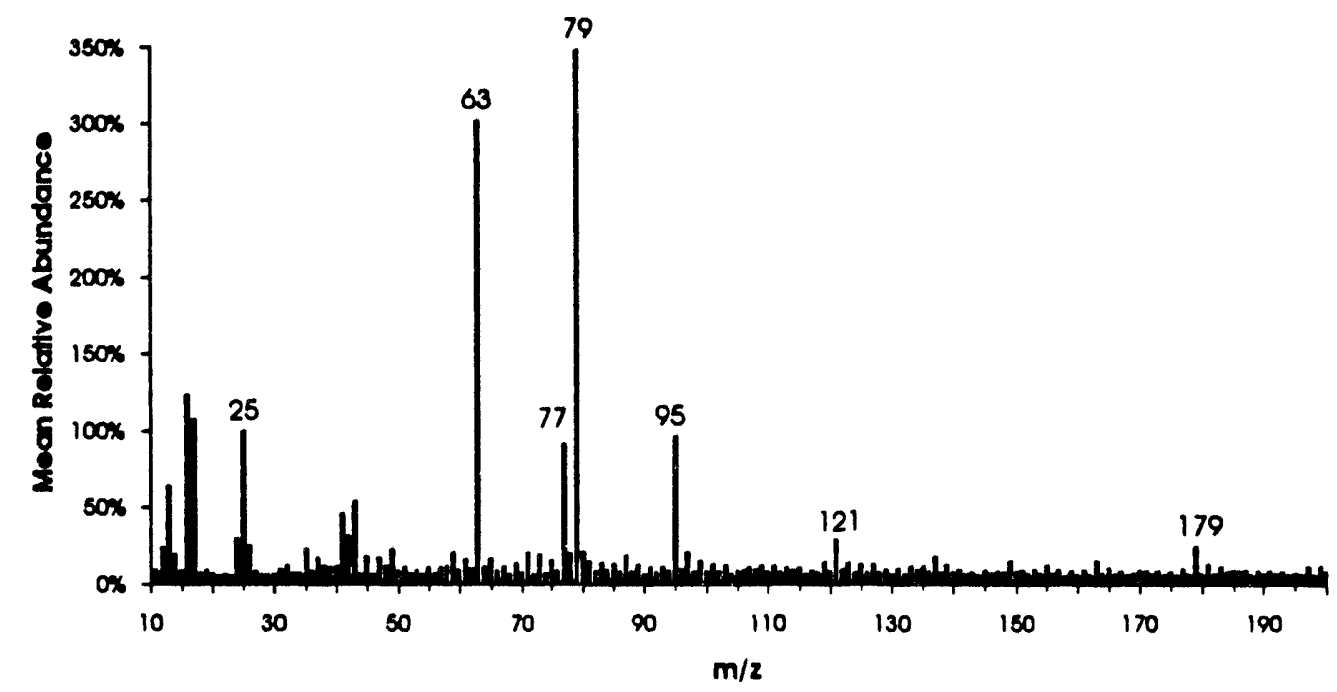

Figure 4. Averaged negative ion spectrum of PMPA on a leaf at $40 \mathrm{ML}$ loading

contaminants in the water or interactions of the water with the leaf. This is important because the AMPAs were deposited on leaves as aqueous solutions. Figure 2 shows an averaged negative ion spectrum of a blank leaf spiked with $5 \mu$ l water; this spectrum is also an average of 8 sets of data taken on 8 different days. Comparing Figures 1 and 2, the major peaks are similar; however, the corresponding abundances are somewhat different. These results are not surprising as MBSA is very sensitive to surface composition, and it would be expected that water-soluble species, such as acids, would be concentrated on the surface as the water evaporated. 
The molecular structures for PMPA, IMPA, and EMPA are shown in Figure 3 along with their molecular weights. The methylphosphonic acid moiety present in all three molecules is responsible for the similar fragmentation chemistries observed in the anion SIM spectra.

The averaged negative ion spectra for PMPA, IMPA, and EMPA on plant leaves are shown in Figures 4, 5, and 6, respectively. The AMPA spectra were taken for leaves spiked at $40 \mathrm{ML}$ and are an average of 8

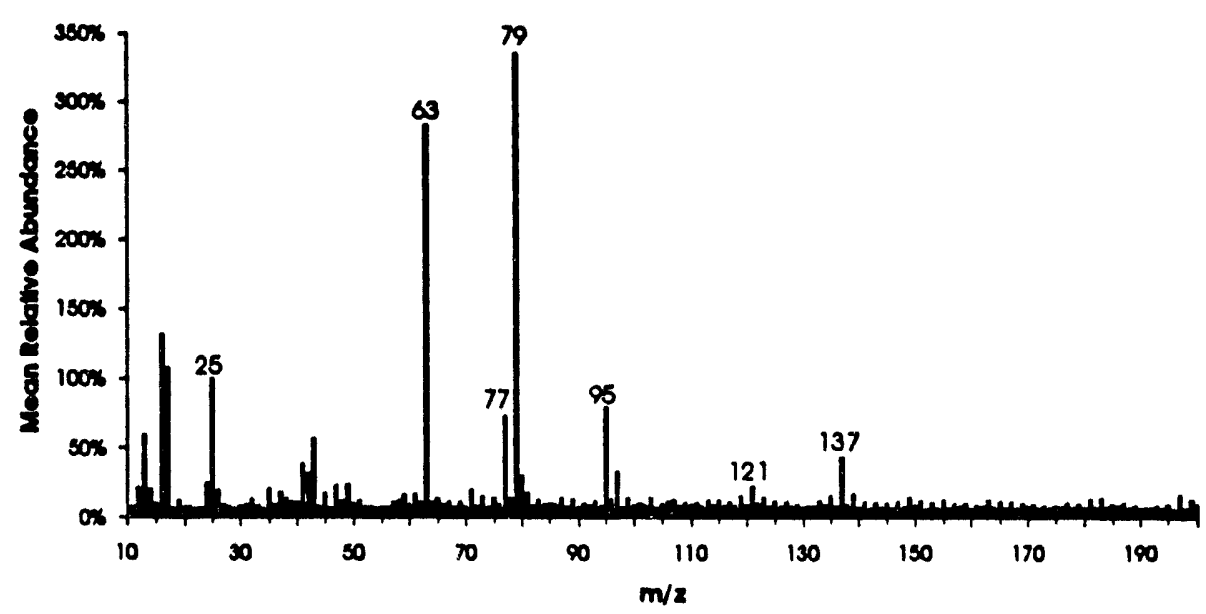

Figure 5. Averaged negative ion spectrum of IMPA on a leaf at $40 \mathrm{ML}$ loading.

sets of data. Comparing the three spectra, the $[\mathrm{M}-\mathrm{H}]^{-}$ions at $179^{-}$(PMPA), 137- (IMPA), and 123(EMPA) standout as the only major differences.

The fragment ions at $121^{\circ}, 95^{\circ}, 79^{\circ}, 77^{\circ}$, and $63^{\circ}$ marked on the spectra are identified in Figure 7 . The $79^{\circ}$ and $63^{\circ}$ ions are the most abundant ions observed in the negative ion spectra and are attributed to $\mathrm{PO}_{3}{ }^{-}$and $\mathrm{PO}_{2}^{-}$. These ions are commonly observed from compounds containing phosphorous and oxygen

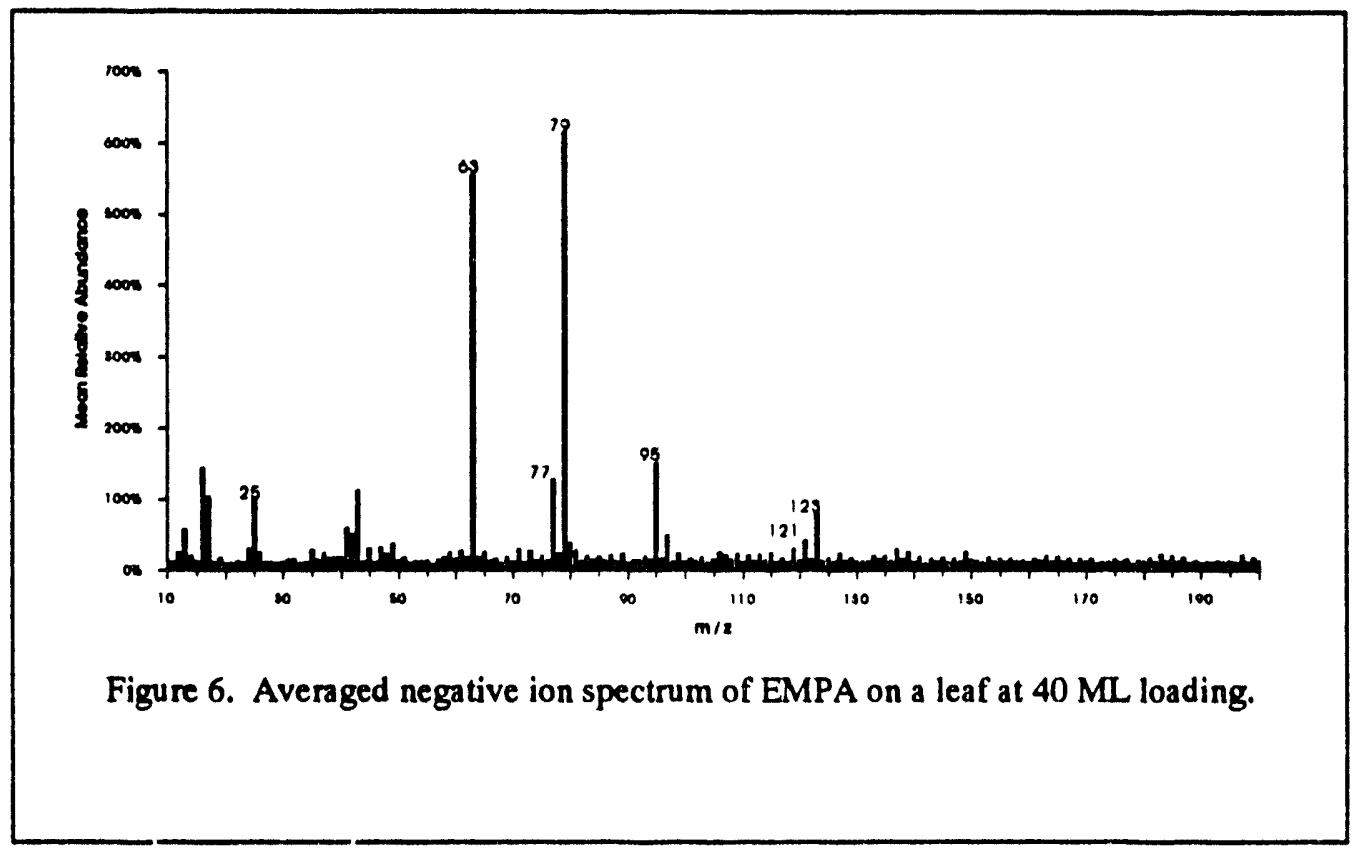


(recall a $79^{-}$peak is observed as a major peak in the background spectra). The $95^{-}$ion is interpreted as the methyl phosphonate ion which would arise from cleavage of the large alkyl groups from the parent molecules. Another fragment ion indicative of the methyl phosphonate moiety is observed at $\mathrm{m} / \mathrm{z} 77^{-}$, which is believed to bave the structure presented in Figure 7. It is speculated that $77^{-}$is formed by dehydration of the $95^{\circ}$, or by loss of an alcohol from the $[\mathrm{M}-\mathrm{H}]^{-}$ion. The ion observed at $\mathrm{m} / \mathrm{z} 121^{-}$in the spectra of all three compounds is believed to be an impurity or secondary degradation product in the AMPA solutions as we bave not seen it in newer sets of solutions. This ion would correspond to the loss of $\mathrm{C}_{4} \mathrm{H}_{10}$ from [PMPA-H], $\mathrm{CH}_{4}$ from [IMPA-H], and $\mathrm{H}_{2}$ from [EMPA-H]". Two structures are proposed in Figure 7. Continued<smiles>C=COP(C)(=O)[O-]</smiles><smiles>O=P1([O-])CCCO1</smiles>

$121^{\circ}$ $95^{\circ}$<smiles>CP(=O)([O-])O</smiles><smiles>O=P(=O)[O-]</smiles>

$79^{\circ}$<smiles>CP(=O)([O-])O</smiles>

77

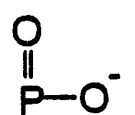

$63^{\circ}$

Figure 7. Fragment ions from AMPA spectra.

investigations as to the origin of this peak are being pursued.

\section{Detection Limits}

The detection limits of MBSA for AMPAs using the quadrupole MBSA instrument were determined by collecting spectra from plant leaves spiked with the AMPAs at three different loadings. These data were collected on 8 separate days; the results were statistically analyzed to determine the reproducibility of the measurements. The intensity and precision of background signal clearly bave important roles in the detection of AMPAs. In order to quantitatively characterize the background, the relative standard deviation was determined for each $\mathrm{m} / \mathrm{z}$ value in all the data sets. The relative standard deviation was determined by calculating the standard deviation at a given $\mathrm{m} / \mathrm{z}$ value from 8 data sets and dividing by the mean value of the relative abundance at that $\mathrm{m} / \mathrm{z}$ value. (See Appendix II-A for a more detailed description of the statistical analysis used in these studies.)

In Figure 8, the relative standard deviations of data taken from the blank leaf are shown plotted versus the $\mathrm{m} / \mathrm{z}$ values. The results indicate that the relative standard deviations are independent of $\mathrm{m} / \mathrm{z}$ value. In Figure 9, the relative standard deviations of data taken for $40 \mathrm{ML}$ PMPA on a leaf are shown plotted versus the $\mathrm{m} / \mathrm{z}$ values. By comparing Figures 8 and 9 , it is concluded that no increase in uncertainty of the measurement is observed with the addition of the analyte.

In order to evaluate these results by data set, the relative standard deviations were averaged over the range which the data was collected (m/z 10 to 200); the results are tabulated in Table 2 . These results indicate that the average relative standard deviations for the different data sets are similar, ranging from $64 \%$ to 97\%. 


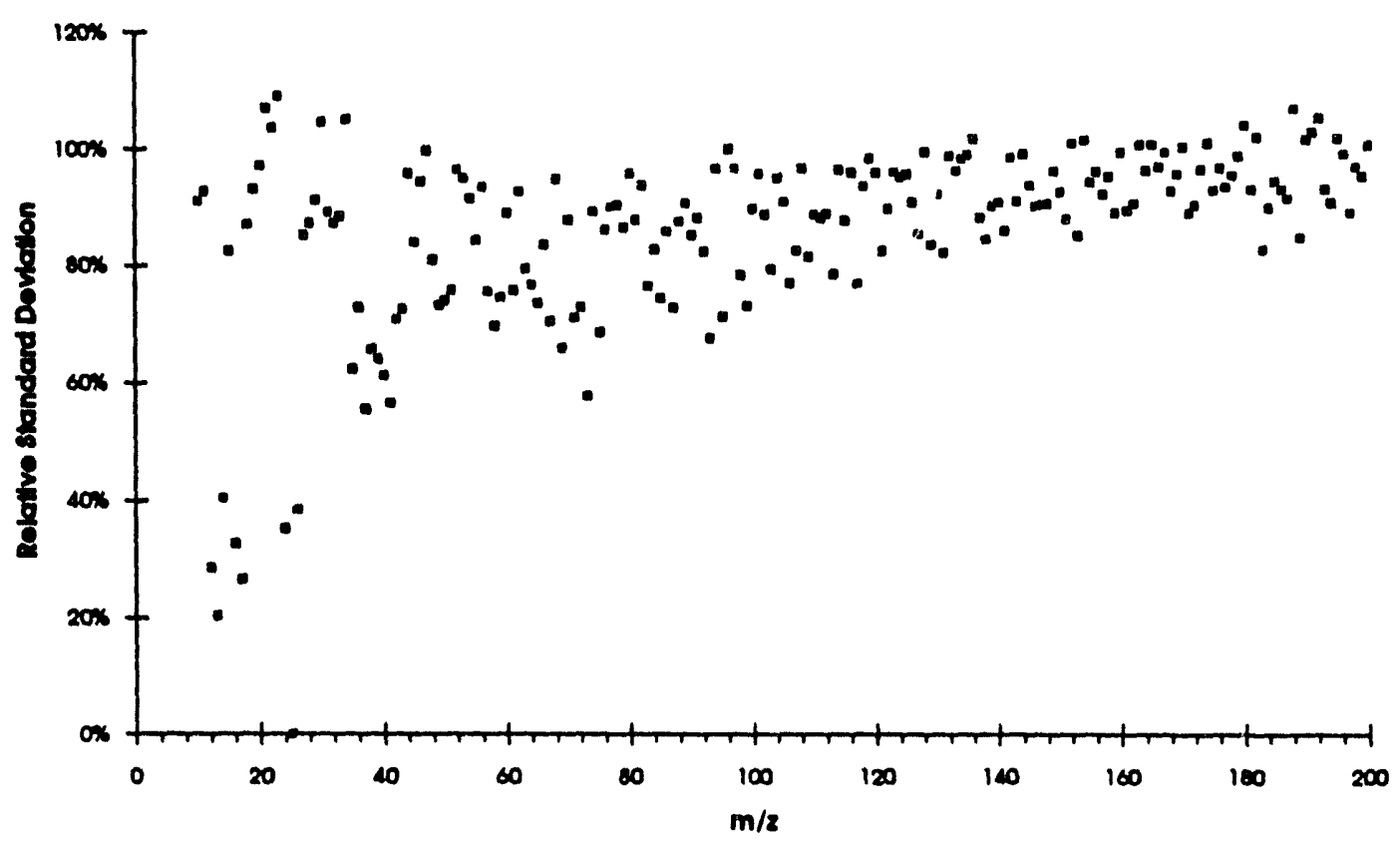

Figure 8. Relative standard deviation versus $\mathrm{m} / \mathrm{z}$ for a blank leaf.

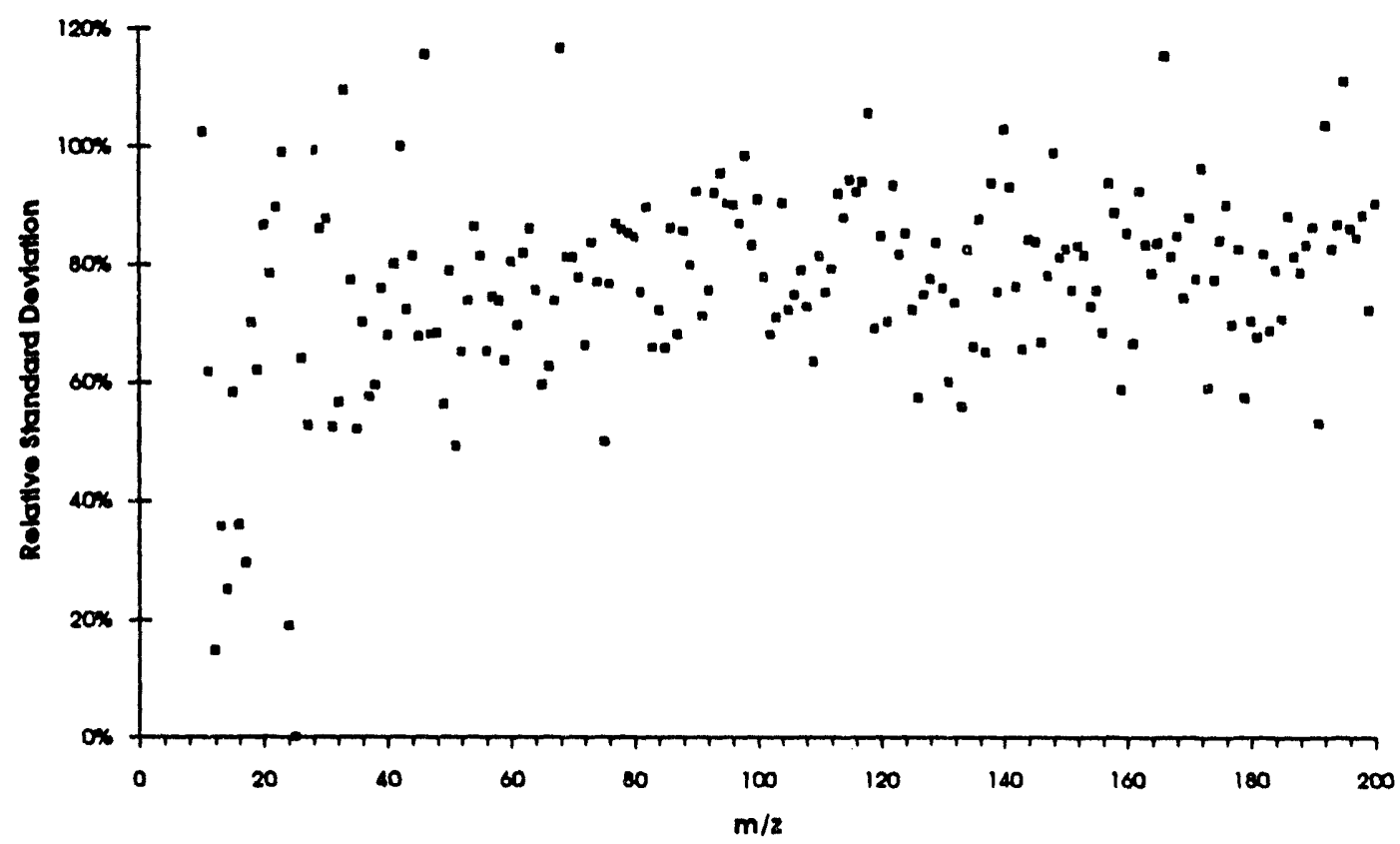

Figure 9. Relative standard deviation versus $\mathrm{m} / \mathrm{z}$ for $40 \mathrm{ML}$ PMPA on a leaf. 
Table 2. Average Standard Deviations From AMPA Measurements

\begin{tabular}{|c|c|c|c|}
\hline Analyte & Loading (ng) & $\begin{array}{c}\text { Maximum Surface Coverage } \\
\text { (monolayers) }\end{array}$ & $\begin{array}{c}\mathbf{\sigma}_{\mathbf{a}} \text { [Ave. Rel. } \\
\text { Std. Dev. (\%) }\end{array}$ \\
\hline PMPA & 500 & 40 & 77 \\
\hline PMPA & 50 & 4.0 & 70 \\
\hline PMPA & 5 & 0.4 & 81 \\
\hline & & 40 & 64 \\
\hline IMPA & 500 & 4.0 & 97 \\
\hline IMPA & 50 & 0.4 & 75 \\
\hline IMPA & 5 & & 88 \\
\hline & & 40 & 67 \\
\hline EMPA & 500 & 4.0 & 80 \\
\hline EMPA & 50 & 0.4 & \\
\hline EMPA & 5 & & \\
\hline & & & \\
\hline Leaf & na. & & \\
\hline
\end{tabular}

In order to determine the lower limit of detection, a criterion was set to evaluate the peak abundances. If the peak abundance was greater than the background plus $2 \sigma_{\mathrm{a}}$ (where $\sigma_{\mathrm{a}}=$ averaged standard deviation over the entire spectrum, see Appendix A), the peak was deemed significant. The background was calculated by averaging the signal in the $\mathrm{m} / \mathrm{z}$ range of 165 to 175 . This region was chosen because it lacked variable spectral features and is representative of much of the rest of the background in the negative ion spectrum.

Histograms representing the mean relative abundances for the major fragment ion peaks at $63^{-}, 77^{-}, 79^{-}$, 95", and [M-H] ${ }^{-}$are shown in Figures 10-12 for PMPA, IMPA, and EMPA, respectively, on leaves at the three different loadings. The discrimination level described above is also shown. These figures clearly show a decrease in signal with decrease in AMPA loading.

At the $40 \mathrm{ML}$ and $4 \mathrm{ML}$ loadings for all three analytes, the following fragments readily passed this requirement: 63-, 79-, and 95-. As noted in Figure 6, the 95- fragment is unique to the AMPAs, and along with the $63^{-}$and $79^{-}$peaks, identify the presence of an AMPA on the leaf surface. These peaks, though, are not selective for the individual AMPA. Selectivity is possible using the $[\mathrm{M}-\mathrm{H}]^{-}$peaks: the abundances of these peaks, $179^{-}$for PMPA, $137^{-}$for IMPA, and $123^{-}$for EMPA, are just above the discrimination level at the $40 \mathrm{ML}$ loading but do not pass the requirement at the $4 \mathrm{ML}$ loading. The 
signal-to-noise ratios for the [M-H] ${ }^{-}$ions at the $4 \mathrm{ML}$ loading are just above 2 ; however, due to the uncertainty in the measurement, the abundances of these peaks are not acceptable to determine a positive

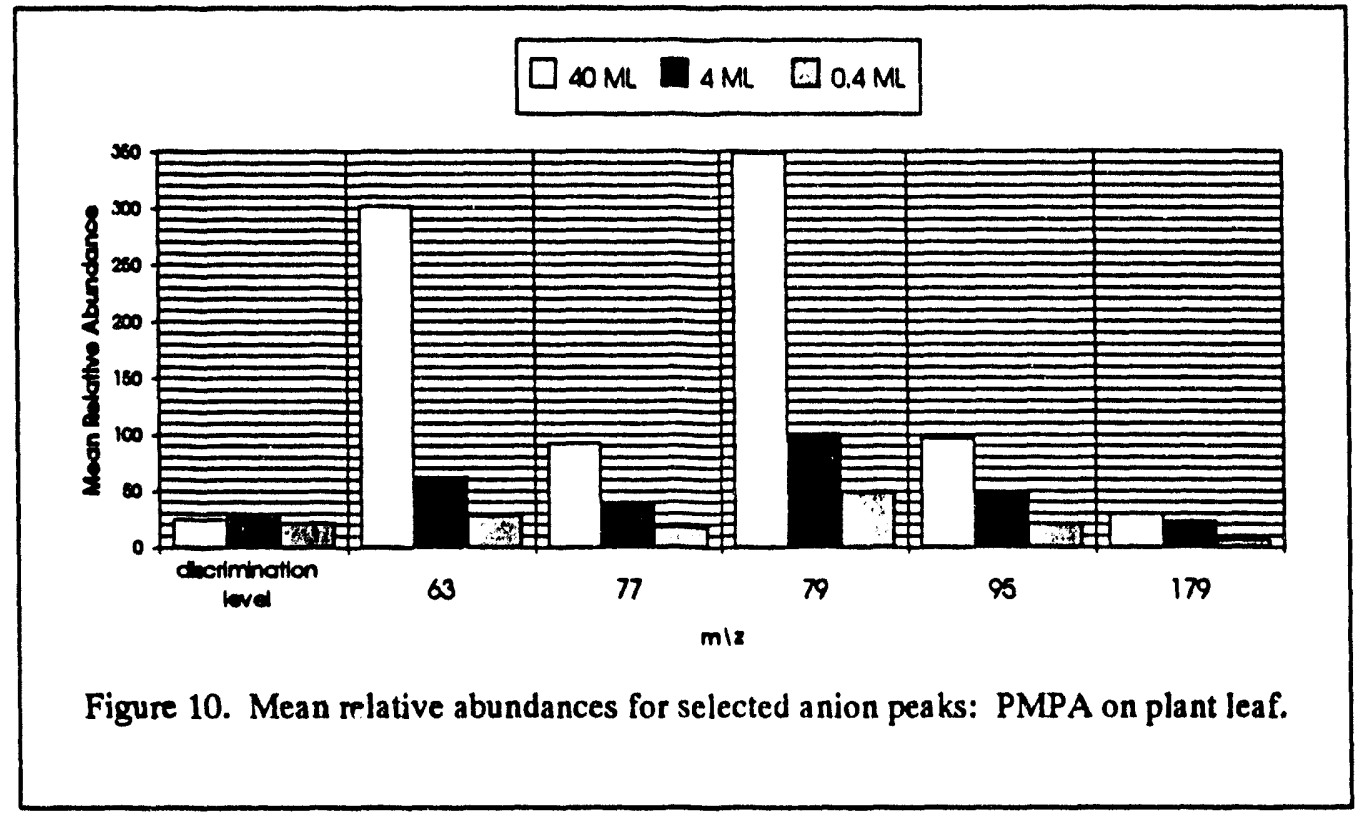

response. These results indicate that the $40 \mathrm{ML}$ loading of PMPA, IMPA, or EMPA on plant leaves can be selectively detected using MBSA with quadrupole based MS. At a loading of $4 \mathrm{ML}$, the AMPAs can be detected; however, selective detection of the individual AMPAs is not practical.

At the 0.4 ML loading for PMPA, the $79^{-}$and $63^{-}$peaks fulfills the above requirement. At the $0.4 \mathrm{ML}$.

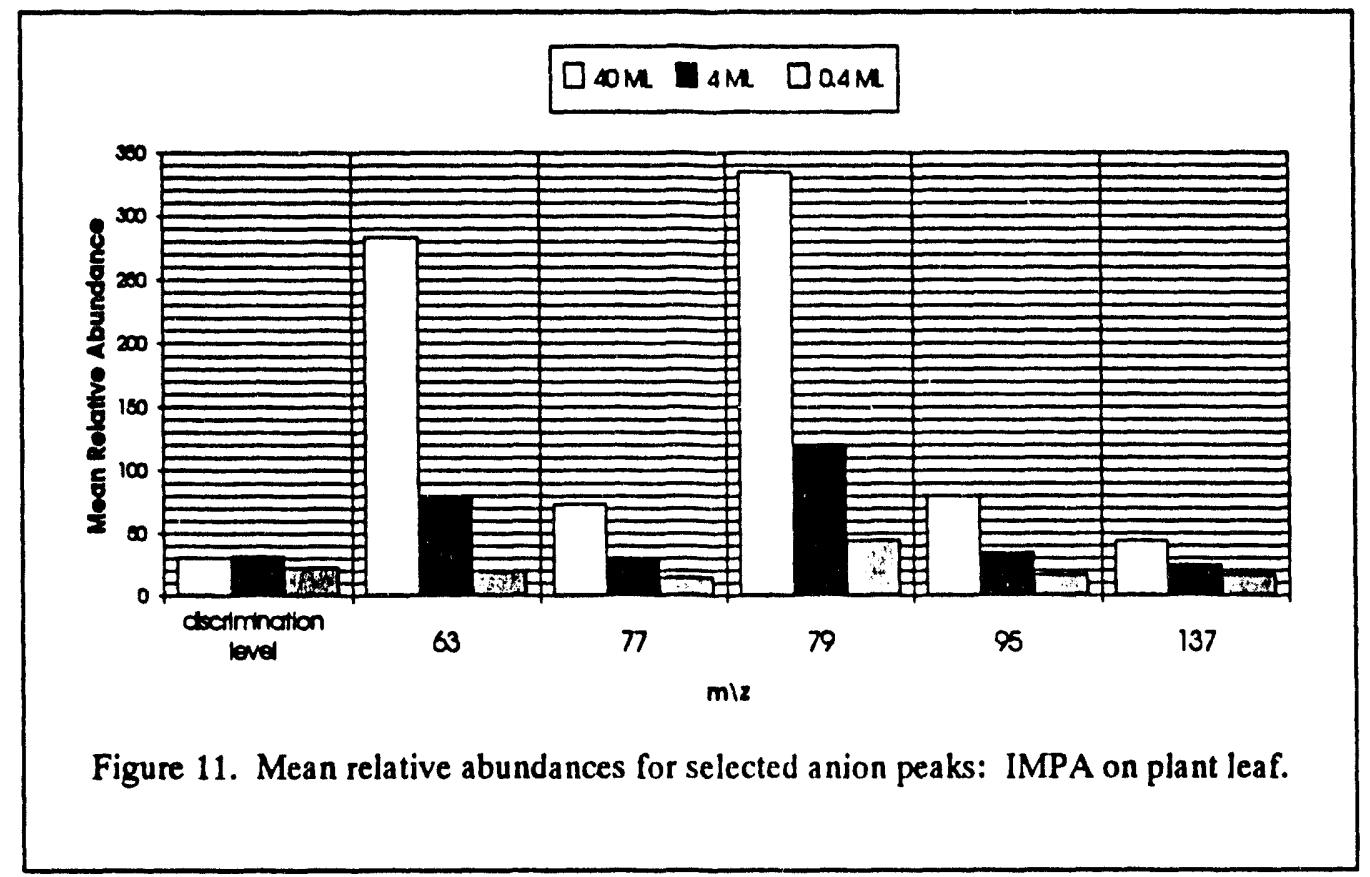

loading for IMPA and EMPA, only the 79- peak is above the discrimination level. Additionally, at these loadings for all three analytes, background peaks at $80^{\circ}$ and $97^{\circ}$ become major peaks in the spectra as demonstrated in Figure 13 which shows the averaged negative ion spectrum of 0.4 ML PMPA on a leaf (see Figure 5 for background spectrum). Due to the common 79- peak in the background and the AMPA 


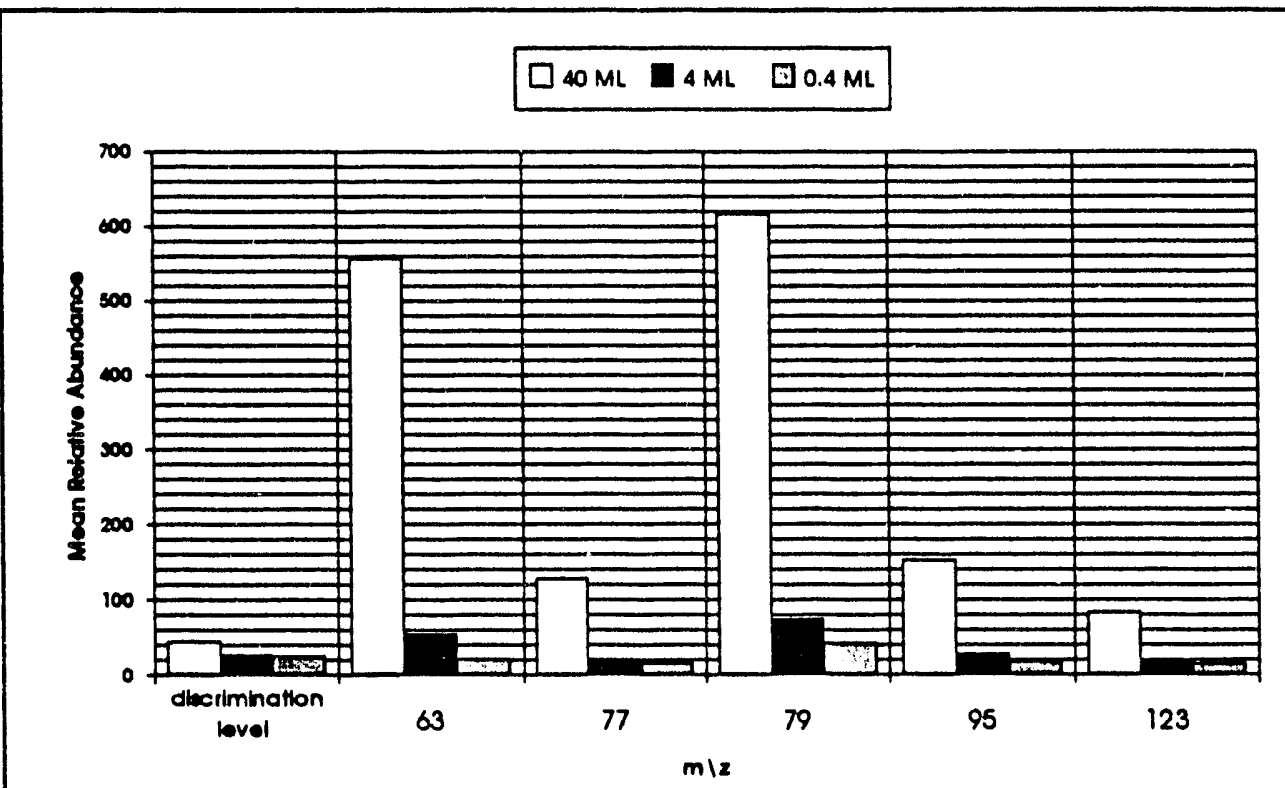

Figure 12. Mean relative abundances for selected anion peaks: EMPA on plant leaf.

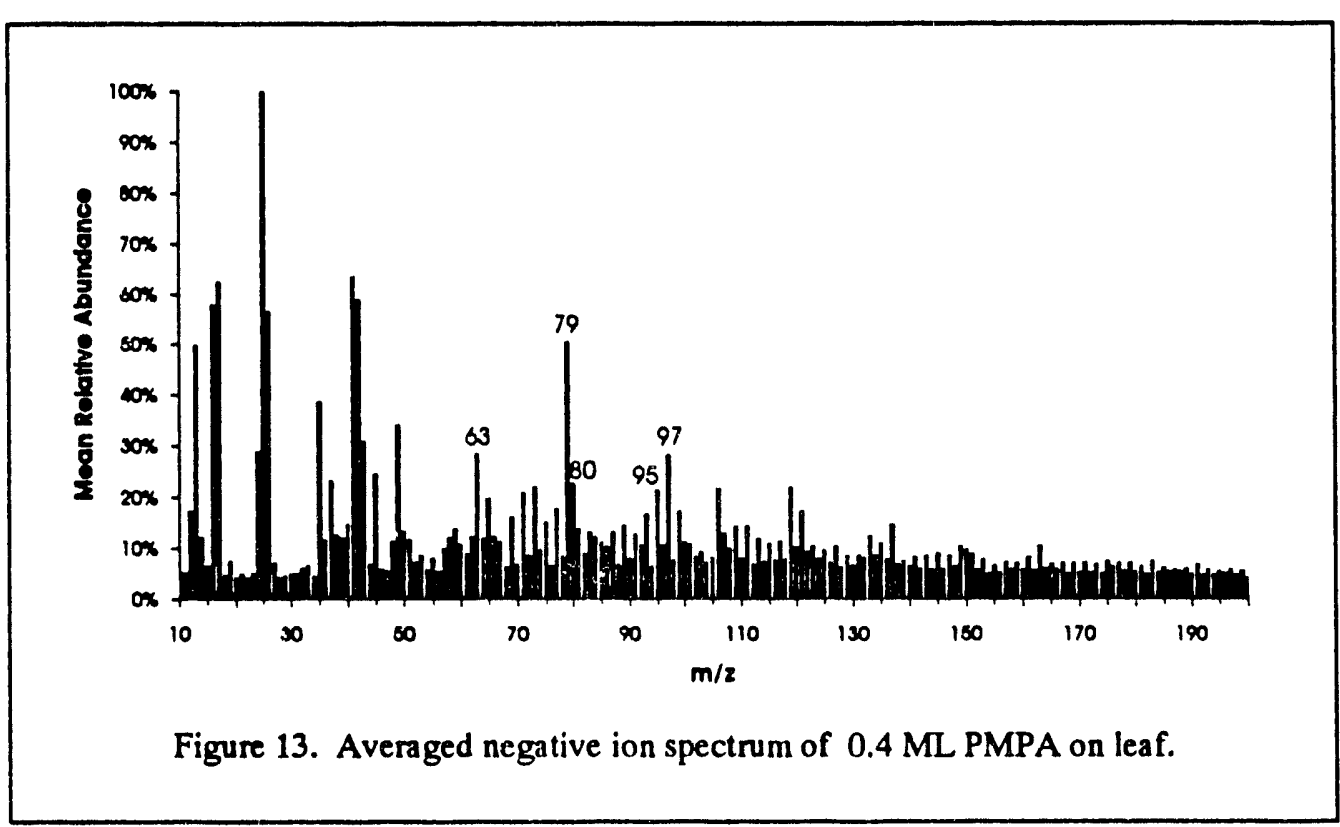

spectra, this peak cannot be assigned to AMPA. These results indicate that, as we expected, the $0.4 \mathrm{ML}$ loading of PMPA, IMPA, and EMPA is below the detection level of the quadrupole MBSA instrument. 


\section{Conclusions}

The lower limit of detection for AMPAs on plant leaves by quadrupole MBSA was determined to be 4 ML. At this level, a sequence of peaks assigned to anion fragments of the AMPA molecules are observed at conclusive levels above the background. The [M-H] $]^{-}$peaks, which provide selectivity among the AMPAs, are not conclusive at this level. At the $40 \mathrm{ML}$ loading, the AMPAs on plant leaves were found to be selectively detectable as the $[\mathrm{M}-\mathrm{H}]^{-}$peaks are above the discrimination level. The limit of detection was determined to be constrained by the chemical noise level of the background. In order to lower the detection levels, a reduction in the chemical noise level is necessitated.

These results also corroborate previous work for AMPA detection on dandelion leaves. The relative abundances for fragment ions associated with the AMPAs on the house plant leaves compare well to those observed from AMPAs on dandelion leaves, as demonstrated in Figures 14 and 15 . Further studies were conducted to determine if the type of plant leaf chosen would have a significant effect; the results of this study are presented in section IV of this report. Based on our experience to date we anticipate MBSA to be broadly applicable to detection of AMPAs on leaves.

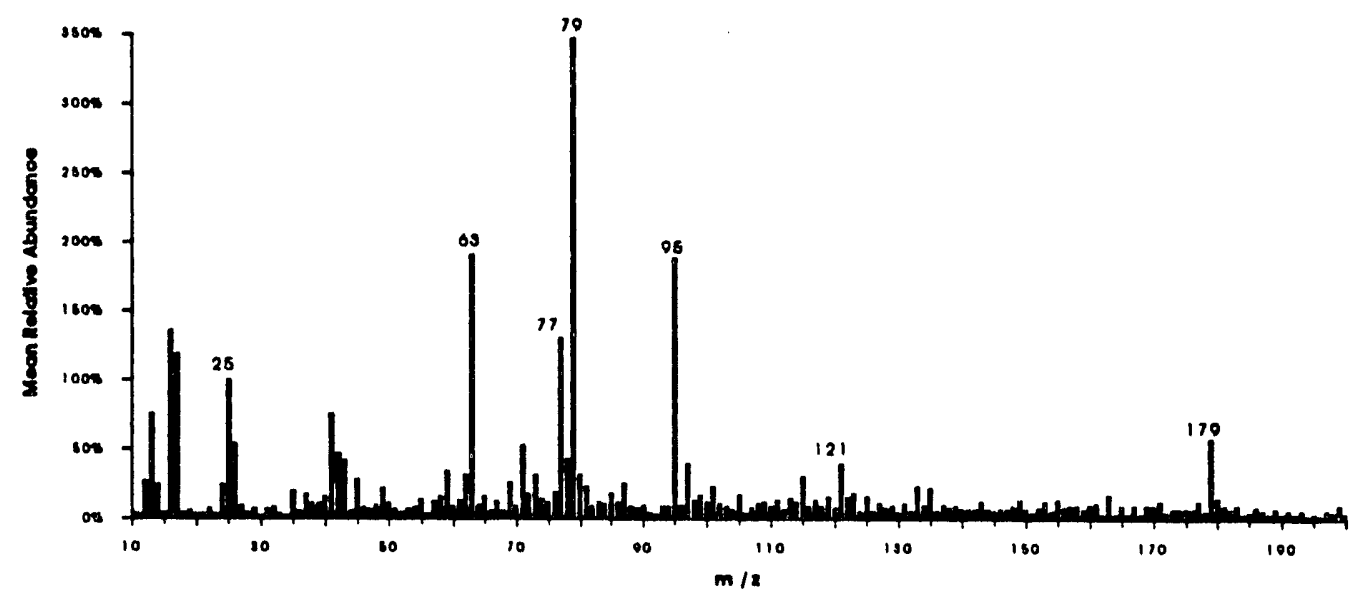

Figure 14. Negative ion spectrum of 16 ML PMPA on a dandelion leaf.

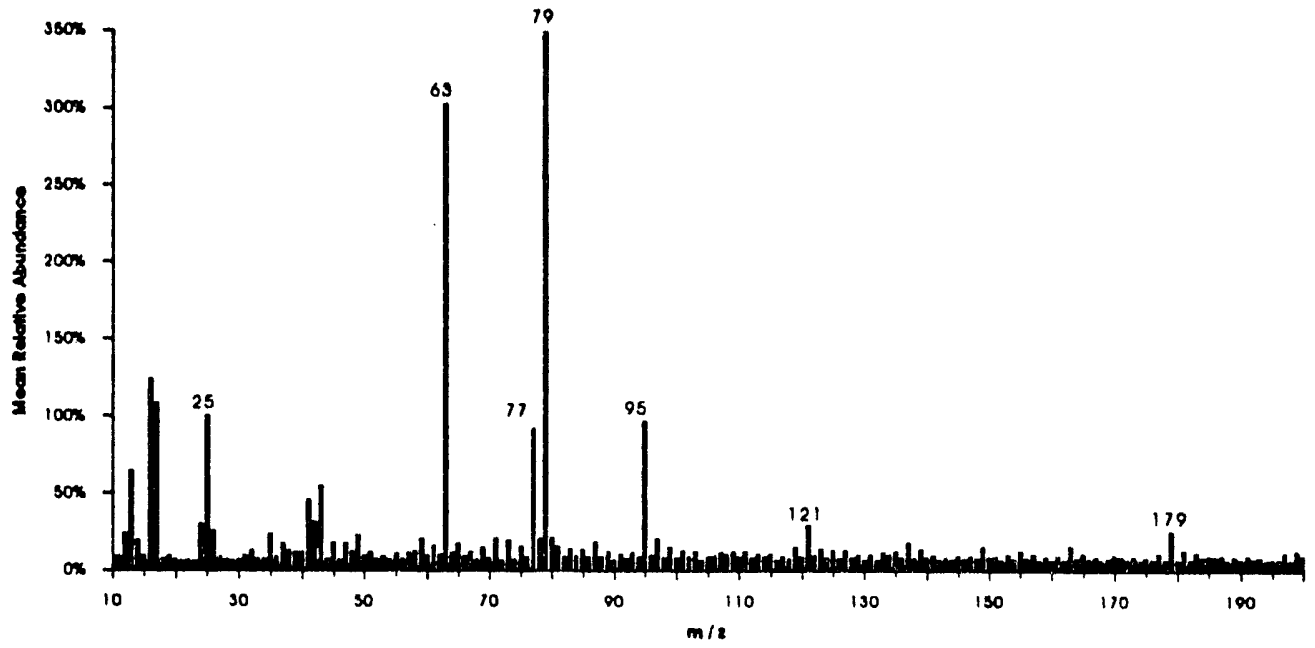

Figure 15. Averaged negative ion spectrum of 40 ML PMPA on a house plant leaf. 


\section{Significance to Direction of Current Work}

These results demonstrate the advantages of a method that could selectively detect the AMPAs in the presence of a chemical noise background. This issue is currently being addressed through the development of the ion trap based MBSA instrument. We believe we will obtain better specificity with the ion trap system by utilizing its mass spectrometry/mass spectrometry capability.

Due to the differences in the quadrupole and ion trap based instruments, the limits of detection determined with the quadrupole system are believed to be conservative estimates of the detection levels that will be achieved with the ion trap instrument. These measurements will be used as a benchmark in determining the improved sensitivity and specificity possible with the ion trap based system.

\section{Appendix II-A}

\section{Relative Standard Deviation:}

The relative standard deviations for each data set were determined in the following manner:

1. Standard deviations were determined for the relative abundances at each $\mathrm{m} / \mathrm{z}$ value for each data set. The abundances were normalized to the $25^{-}$peak in each spectrum to obtain the relative abundance. For example, in the $40 \mathrm{ML}$ PMPA on leaf data set, at $\mathrm{m} / \mathrm{z} 50^{-}$, the relative abundances were: $7,6,4,8,6,4,16$, and 25 . The standard deviation for these relative abundances is 7 .

2. Mean relative abundances at each $\mathrm{m} / \mathrm{z}$ value were determined for each data set. For the same example listed above, the mean relative abundance is 9.5 .

3. Relative standard deviations were determined by dividing the standard deviation at each $\mathrm{m} / \mathrm{z}$ value by the corresponding mean relative abundance. For the same example listed in steps 1 and 2 , the relative standard deviation is $74 \%$.

\section{Average Relative Standard Deviation:}

The average relative standard deviations for each data set were determined by taking the mean of the relative standard deviations over the entire range of $\mathrm{m} / \mathrm{z}$ values (10 to 200).

\section{Discrimination Level:}

The discrimination levels for the relative abundances used in determination of the detection level were determined in the following manner:

1. The average standard deviation was determined by taking the mean of the standard deviations of the relative abundances for a given data set over the entire range of $\mathrm{m} / \mathrm{z}$ values. (See step 1 in the Relative Standard Deviation section above for determination of the standard deviation.)

2. The background from the averaged negative ion spectrum was determined by averaging the abundances in the $\mathrm{m} / \mathrm{z}$ range of 165 to 175 .

3. The discrimination level is equal to 2 times the average standard deviation plus the background. 
This Page Intentionally Left Blank 


\section{Chemical Interferences}

\section{Detection of AMPAs in the Presence of Herbicides and Pesticides}

\section{Introduction}

A significant challenge to the unambiguous detection of $\mathrm{CW}$ degradation products is the presence of a wide variety of pesticides and herbicides in the environment. These compounds have chemical structures that are similar to those of the $\mathrm{CW}$ degradation products. To evaluate the potential for interferences from these types of environmental sources we have chosen a set of pesticides and herbicides that are commonly used throughout the world for testing with the alkylmethylphosphonic acids (AMPA). The objectives of this investigation were to determine if the AMPAs could be detected in the presence of these interferences, the potential for false positive detections, and to provide data that could be used to evaluate the potential for methods for reducing false positives.

\section{Experimental}

All measurements were performed using the quadrupole mass spectrometer based molecular beam surface analysis instrument. Positive and negative ion spectra were obtained simultaneously in all measurements. Measurements were made using plant leaves (dandelion) and stainless steel samples. The plant leaves were grown in a protected environment without exposure to herbicides or pesticides. The stainless steel samples were washed with soap and water, rinsed with distilled water (nanopure), sonicated in methanol and then methylene chloride. We first obtained and evaluated the secondary ion mass spectra of the compounds in their "pure" form in order to establish baseline spectra. The degradation product of the agent GD, pinacolyl methylphosphonic acid (PMPA), was chosen for this study because it is very representative of the general class of AMPAs. The protocol for the mixed analysis was to first spike a sample surface with the pesticide or berbicide (in solution), dry it in air, analyze it in the MBSA instrument, remove the sample from the instrument and add a spike of the PMPA, dry in air, and then again analyze with MBSA. Table 1 provides the common name of the compounds, their structure and molecular weight, the amount added to the samples, and the ratio of interferent/PMPA.

\section{Results}

\section{Single Component Experiments}

PMPA: The secondary ion mass (SIM) spectra of PMPA on a dandelion leaf and on stainless steel are shown in Figure 1. The anjon spectra contains the $\mathrm{m} / \mathrm{z} 179^{-}$molecular ion peak, and the primary fragmentation ions at $\mathrm{m} / \mathrm{z} 95^{\circ}, 79^{-}, 77^{\circ}$, and $63^{\circ}$. It is also interesting to note that on the stainless steel substrate, a peak at $\mathrm{m} / \mathrm{z} 97^{+}$is present which is absent on the dandetion surface. This peak may correspond to protonated methyl phosphonic acid, $\left[\mathrm{CH}_{3} \mathrm{P}(\mathrm{OH})_{3}\right]^{+}$.

Pesticides/Herbicides (general): Overall, the SIM spectra for the pesticides/herbicides were much stronger from the stainless steel surface as compared to the dandelion surface. In most cases, higher molecular weight fragment ions were observed and the abundances of the ions were, in general, higher. Additionally, the stainless steel data often displayed fragment ions from both the cation and anion spectra which were attributed to the analyte. In most of the dandelion data, the peaks of interest were observed in the anion spectra. Individual spectra for each of the compounds are provided in Appendix III- $A_{\text {. }}$. 
Table 1 Compounds used in Interference Study

\begin{tabular}{|c|c|c|c|}
\hline Compound & $\begin{array}{l}\text { Structure and Molecular } \\
\text { Weight (daltons) }\end{array}$ & $\begin{array}{c}\text { Amount } \\
\text { Spiked onto } \\
\text { Sample (ng) }\end{array}$ & $\begin{array}{l}\text { Ratio of } \\
\text { Interferent to } \\
\text { PMPA }\end{array}$ \\
\hline $\begin{array}{l}\text { pinacolyl } \\
\text { methylphosphonic } \\
\text { acid in } \mathrm{H}_{2} \mathrm{O}\end{array}$ & 180 & 200 & na \\
\hline $\begin{array}{l}\text { Glyphosate in } \\
\text { methanol }\end{array}$ & 169 & 1550 & 7.75 \\
\hline $\begin{array}{c}\text { Abate in methylene } \\
\text { chloride }\end{array}$ & 466 & 1150 & 5.75 \\
\hline $\begin{array}{l}\text { Dursban in } \\
\text { methanol }\end{array}$ & 350 & 1500 & 7.5 \\
\hline $\begin{array}{l}\text { Fenthion in } \\
\text { methanol }\end{array}$ & 278 & 1250 & 6.25 \\
\hline Mocap in methanol & & 1094 & 5.5 \\
\hline DEF in methanol & & 1000 & 5.0 \\
\hline
\end{tabular}



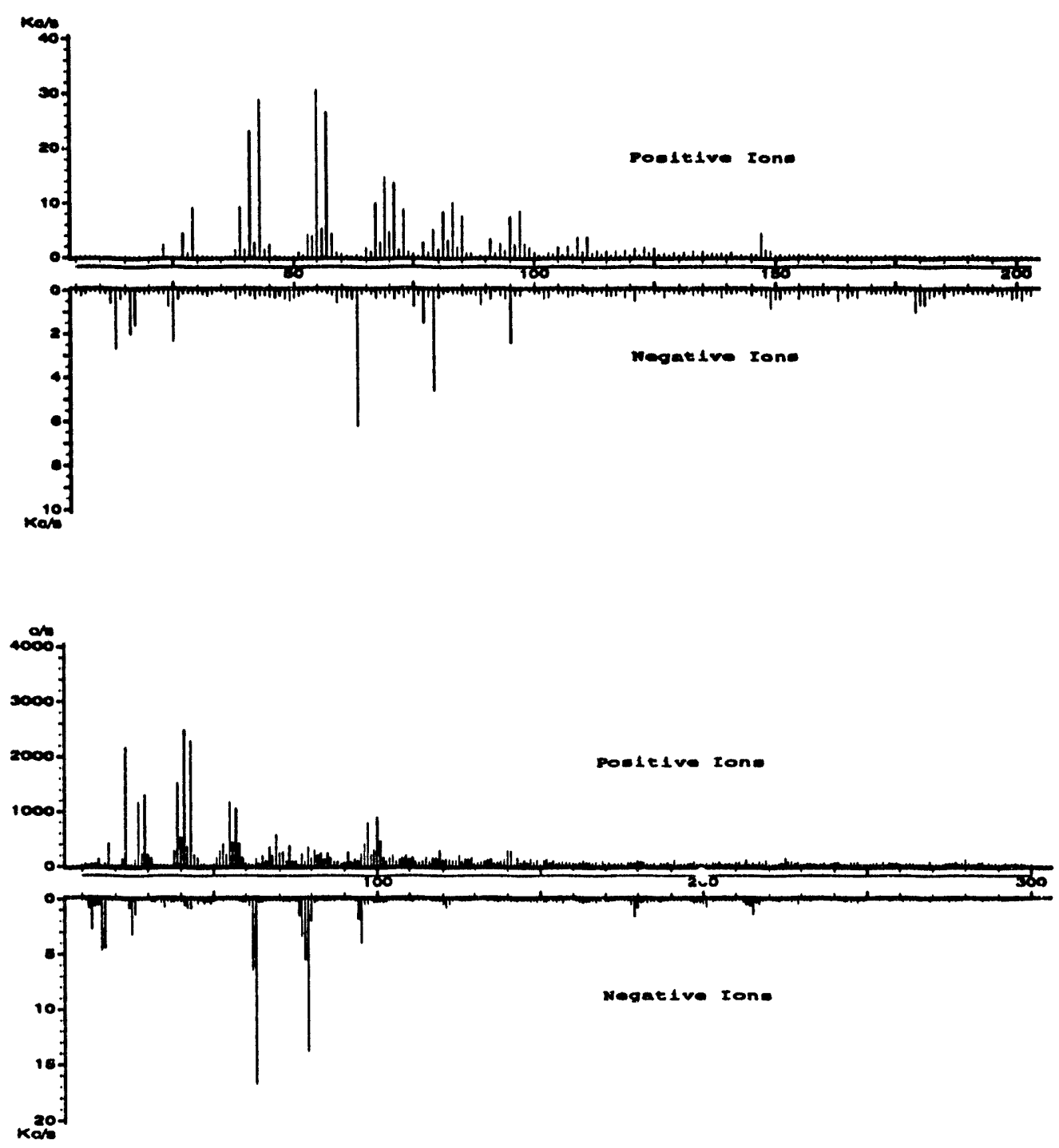

Figure 1. Mass spectra of PMPA on Stainless Steel (bottom) and Dandelion (top).

A few fragment ions were observed in more than one set of data from the pesticides/herbicides. These ions include: $\mathrm{m} / \mathrm{z}=141^{\circ},\left[\left(\mathrm{CH}_{3} \mathrm{O}\right) \mathrm{PSO}\right]^{-}$, seen in abate and fenthion; $\mathrm{m} / \mathrm{z}=127^{\circ}$, most likely $\left[\mathrm{S}_{2} \mathrm{PO}_{2}\right]^{-}$, seen in dursban, mocap, and $\mathrm{DEF}$; and $\mathrm{m} / \mathrm{z}=95^{\circ},\left[\mathrm{SPO}_{2}\right]^{-}$, seen in abate, dursban, fenthion, mocap, and DEF.

It is interesting to note, that a peak at $\mathrm{m} / \mathrm{z}=101^{-}$was present in many of the pesticide/herbicide spectra taken from the dandelion surface. This peak has not been identified, but it was also observed in a study investigating the possible effects of $\mathrm{pH}$ on secondary ion production. In the $\mathrm{pH}$ study it was found that at

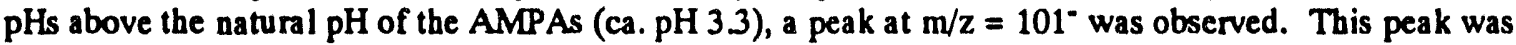
also observed when the leaf was spiked with a $0.01 \mathrm{M} \mathrm{NaOH}$ solution. It was concluded that the 101peak was from the leaf surface and was caused by the application of solutions more basic than $\mathrm{pH}$ 3.3. In the discussion below, it will be noted when the $101^{\circ}$ peak was observed. The following paragraphs summarize the major features of the spectra for each compound. The spectra are contained in Appendix III-A. 
Glyphosate: Very strong spectra were observed from the stainless steel; molecular ions were observed both in the cation spectrum $(\mathrm{m} / \mathrm{z}=170 \text {, (glyphosate }+\mathrm{H}]^{+}$) and anion spectrum $(\mathrm{m} / \mathrm{z}=168$, [glyphosate H]". Glyphosate was of particular interest because it was one of the few compounds to have a phosphonate moiety. Many positive and negative fragment ions were identified. The only possible ions which would interfere in PMPA analysis are at $\mathrm{m} / \mathrm{z}$ values of $79^{\circ}$ and $63^{\circ}$. In the spectra from the dandelion leaf, a very weak molecular ion peak at $\mathrm{m} / \mathrm{z}=168^{-}$was observed $(\mathrm{S} / \mathrm{N} \sim 2)$. A peak at $101^{-}$was observed from tive dandelion surface.

Abate: Very strong spectra were observed from the stainless steel; a molecular ion was observed in the cation spectrum at $\mathrm{m} / \mathrm{z}=466^{+}$, which corresponds to the intact radical cation, [abate $\left.-\mathrm{H}\right]^{+}$. A peak was also observed in the anion spectrum at $\mathrm{m} / \mathrm{z}=450^{\circ}$, but was not assigned. Most of the fragments identified were in the anion spectrum with cation fragments present but unassigned. The highest molecular weight fragment ion observed in the spectra from the dandelion surface was $\mathrm{m} / \mathrm{z}=141^{\circ}$ and identified as $\left[\left(\mathrm{CH}_{3} \mathrm{O}\right)_{2} \mathrm{PSO}^{-}\right.$. Abundant ions were observed at $63^{\circ}, 79^{-}$, and $95^{-}$which correspond to $\mathrm{PS}^{-} / \mathrm{PO}_{2}^{-}, \mathrm{POS}^{-}$ $/ \mathrm{PO}_{3}{ }^{-}$, and $\mathrm{PSO}_{2}{ }^{-}$. These ions are isobaric with ions for the AMPA compounds and illustrate the potential for misidentifying a turget based solely on the presence of these peaks. MS/MS of the parent ion would help to eliminate this potential problem.

Dursban: The highest molecular weight ion observed in the stainless steel spectrum was a cluster of ions at $\mathrm{m} / \mathrm{z}$ values of $196^{+}, 198^{+}$, and $200^{+}$. These fragments are identified as the isotopes of $\left[\mathrm{C}_{5} \mathrm{HNCl}_{3}\right.$ 일, the pattern matches that of a molecule containing three chlorine atoms. Only a few other anion peaks were identified as fragments of dursban. A cation peak is observed at $\mathrm{m} / \mathrm{z}=192^{+}$but was not assigned. The spectra from the dandelion surface has only two peaks which a re attributed to dursban with one being the $192^{+}$peak. Note that the $101^{\circ}$ peak was present in the dandelion anion spectrum.

Fenthion: The stainless steel spectra contain both anion and cation fragments which are attributed to fenthion. A peak at $\mathrm{m} / \mathrm{z}=278^{+}$is observed and presently attributed to the radical cation of fenthion. A peak at $\mathrm{m} / \mathrm{z}=263^{\circ}$ was observed and attributed to the loss of $\mathrm{CH}_{3}$ from fenthion. In addition to the fragment ions identified in both the cation and anion spectra, there were also peaks which may be due to fenthion but are unassigned. The dandelion spectra contain fewer peaks attributed to fenthion fragments than the stainless steel; bowever, in the anion spectra, peaks were present which are also present in the stainless steel data which were not assigned. Note that the $101^{-}$peak was present in the dandelion anion spectrum.

Mocap: In the stainless steel spectra, the only anions observed at strong abundances were at $\mathrm{m} / \mathrm{z}^{-6}$ and $7^{-}$, which may be assigned to either $\mathrm{PS}^{-}$or $\mathrm{PO}_{2}^{-}\left(63^{\circ}\right)$ and to $\mathrm{POS}^{-}$or $\mathrm{PO}_{3}^{-}\left(7^{-}\right)$. In the dandelion data these peaks are also present but at lower abundances. The highest molecular weight peak in the stainless steel data is at $\mathrm{m} / \mathrm{z}=183^{\circ}$, and in the dandelion data at $\mathrm{m} / \mathrm{z}=199^{\circ}$, and $183^{\circ}$. None of the peaks have been assigned. Note that a very small $101^{\circ}$ peak is present in the dandelion anion spectrum. Mocap is the most volatile of all the pesticides/herbicides investigated in this study, which may account for the low number and abundances of peaks.

DEF (s,s,s tributyl phosphorotrithioate): The highest molecular weight ion in the stainless stecl data is at $\mathrm{m} / \mathrm{z}=257^{-}$and is attributed to the loss of $\mathrm{C}_{4} \mathrm{H}$ from DEF. A few other lower mass ions in both the cation and anion spectra were identified as fragments from DEF. It is interesting to note that the presence of cations at $\mathrm{m} / \mathrm{z}=56^{+}$and $52^{+}$. Due to the absence of nitrogen in the DEF molecule, it is not clear if these fragments are radical cations or come from some other contaminant on the stainless steel surface. The dandelion data do not contain these peaks. Anion peaks are observed at $\mathrm{m} / \mathrm{z} 63^{-}$and $79^{-}$, which are isobaric with peaks from AMPAs, as noted for Abate. Note that the $101^{\circ}$ peak was present in the dandelion anion spectrum. 


\section{Results of the Mixed Component Experiments}

General Observations: The results of the mixed experiments (PMPA + pesticide/herbicide) followed the same trends as the single component results discussed above in that the spectra from stainless steel contained peaks of larger masses and the ion abundances were larger compared to data from the dandelion surfaces. In anion spectra collected from the dandelion surfaces, the $101^{-}$peak was not present in any of the mixed system data. It is believed that the $\mathrm{pH}$ of the PMPA solutions is responsible for the absence of this peak.
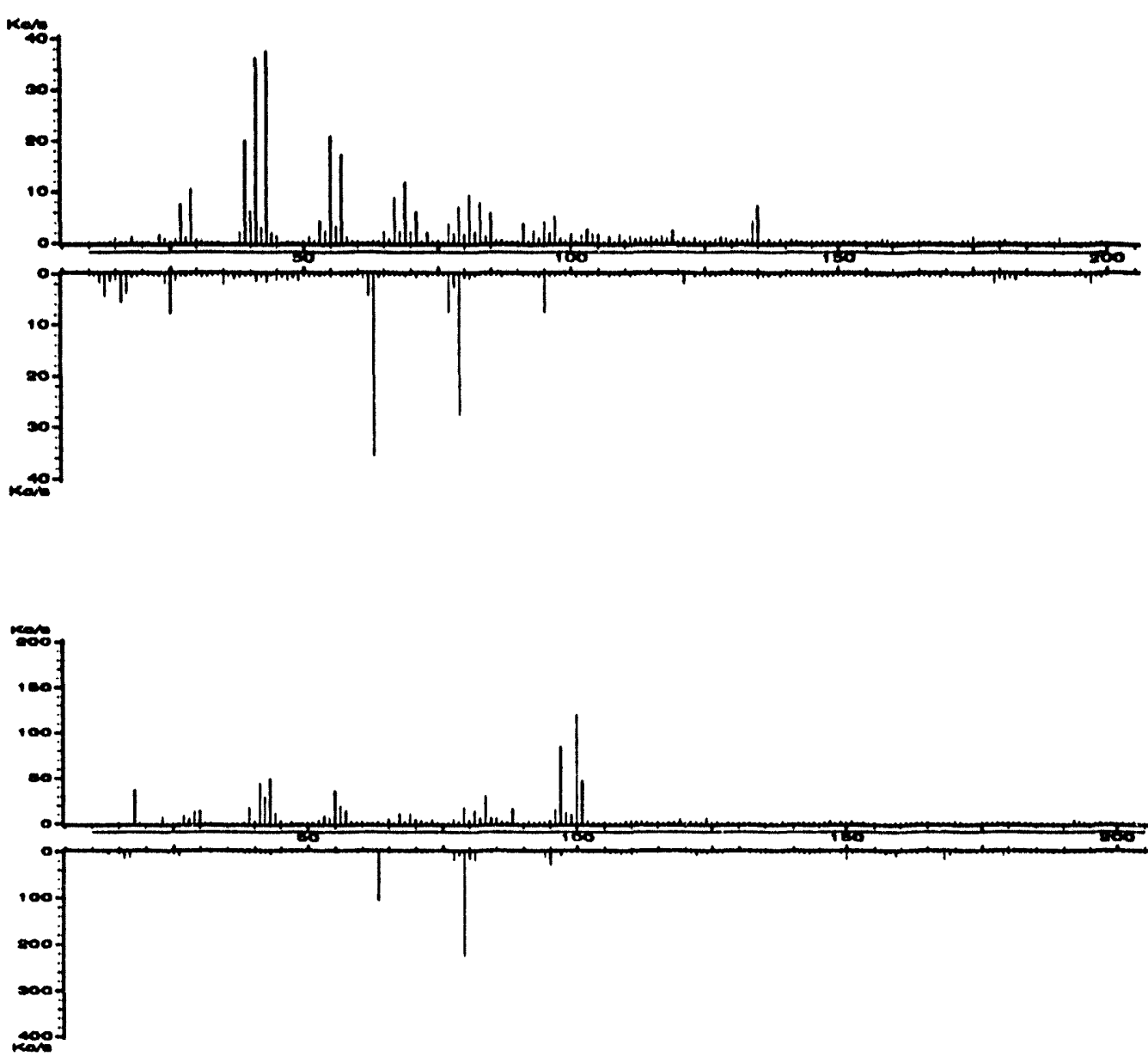

Figure 2. Glyphosate and PMPA mixture on stainless steel (bottom) and dandelion (top).

Glyphosate/PMPA: In the stainless steel data, peaks due to glyphosate are as strong or stronger than those from PMPA. However, the PMPA peaks $\left(179^{\circ}, 121^{\circ}, 95^{\circ}, 79^{\circ}, 77^{\circ}\right.$, and $\left.63^{\circ}\right)$ are observed in the anion spectrum along with the $97^{+}$peak in the cation spectrum. The dandelion spectra display very strong PMPA peaks and no obvious glyphosate peaks. The only interfering peaks in both the stainless steel and dandelion data are the $79^{\circ}$ and $63^{\circ}$ peaks. These data indicate that PMPA may be detected in the presence of glyphosate on either substrate. Since $63^{\circ}$ and $79^{-}$are observed in the spectrum of both glyphosate and PMPA, they are potertial interferents. The presence of both 168- (from Glyphosate) and 179- (PMPA), indicate both compounds are contributing to the $63^{\circ}$ and $79^{-}$peaks. However, $95^{\circ}$ originates only from PMPA, not from Glyphosate, and is thus a good indicator. The optimum solution is to perform MS/MS on the $179^{-}$peak. 
Abate/PMPA: In the stainless steel data, peaks due to abate are as strong or stronger than those from PMPA. The PMPA peaks $\left(179^{\circ}, 121^{\circ}, 95^{\circ}, 79^{\circ}, 77^{\circ}\right.$, and $\left.63^{\circ}\right)$ are observed in the anion spectrum along with the $97^{+}$peak in the cation spectrum. The ion abundances in the data taken from the dandelion surface are weaker than those in the stainless steel data. Peaks identified as abate and PMPA fragments are observed in the dandelion spectra. The molecular ion for PMPA $\left(m / 2=179^{-}\right)$is weak $(s / n-3)$ but detectable. In the case of abate, the interfering peaks in both the stainless steel and dandelion dats are the

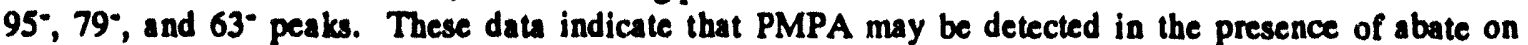
stainless steel. Detection of PMPA on dandelion surfaces in the presence of abate may be more difficult due to the low abundances of the $179^{\circ}$ peak and the interferences at $95^{\circ}, 79^{\circ}$, and $63^{\circ}$.
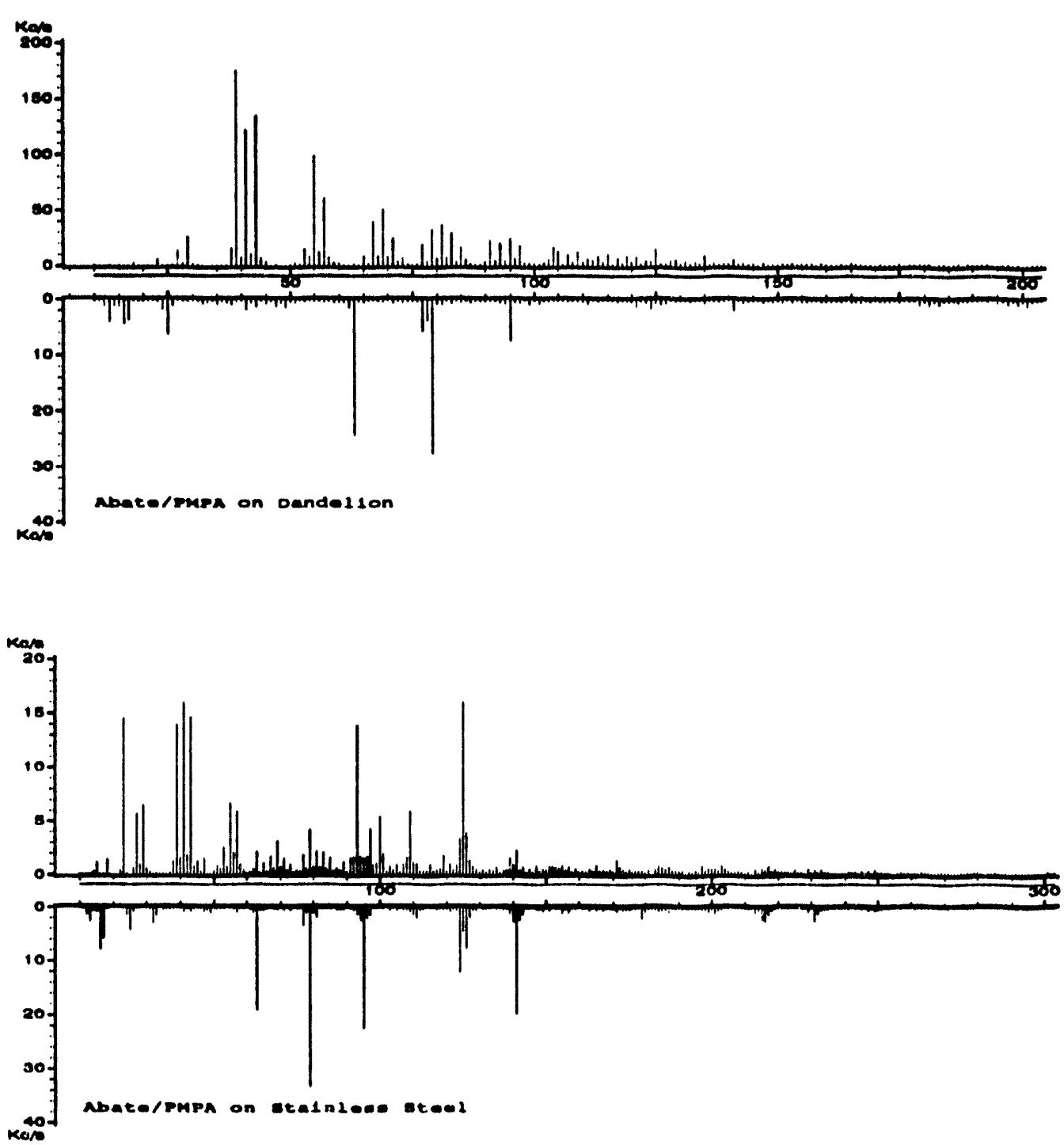

Figure 3. Abate and PMPA mixture on stainless steel (bottom) and dandelion (top). 

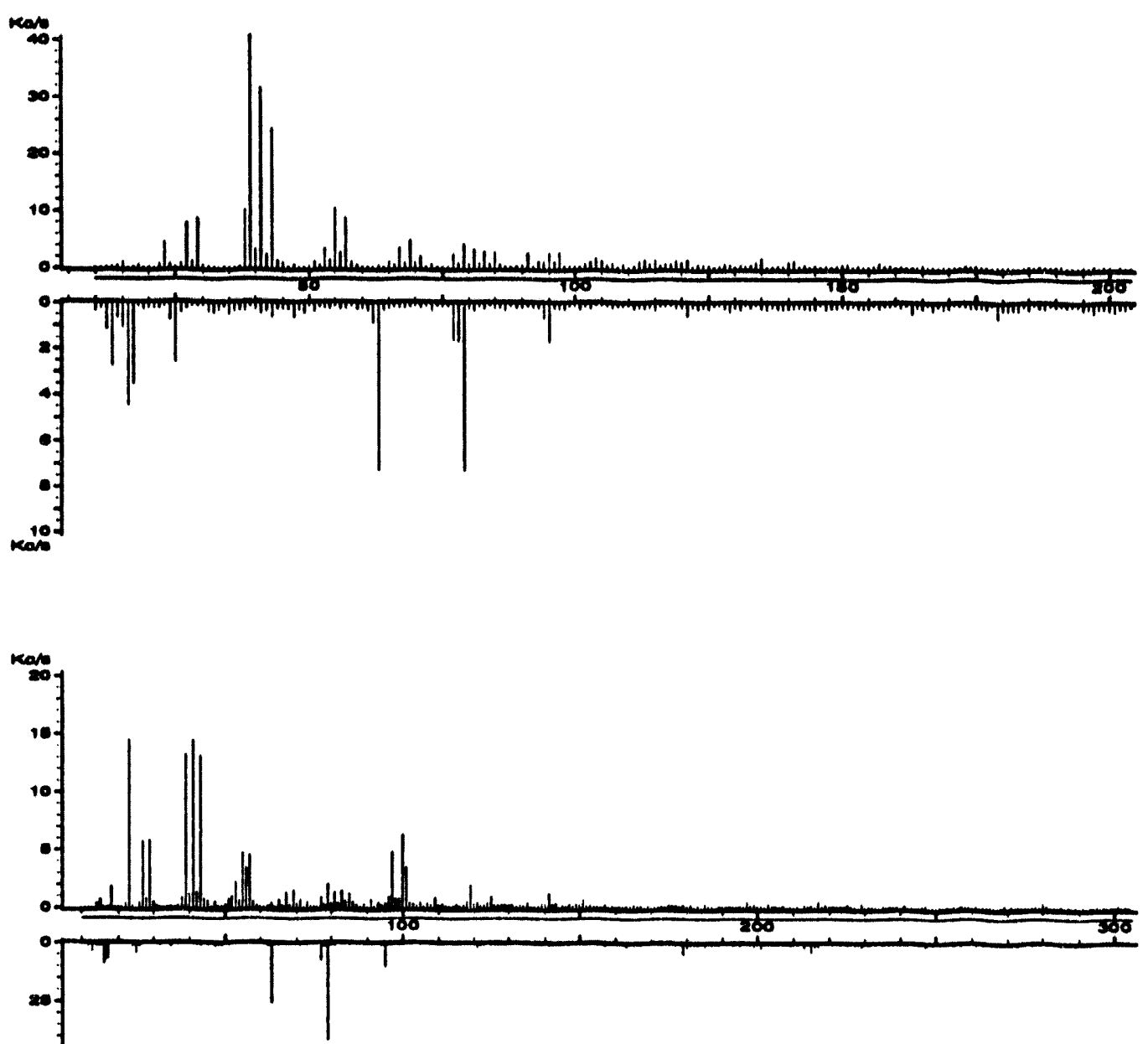

ro

Figure 4. Dursban and PMPA mixture on stainless steel (bottom) and dandelion (top).

Dursban/PMPA: In the stainless steel data, peaks due to PMPA are stronger than those due to dursban. The PMPA peaks $\left(179^{\circ}, 121^{\circ}, 95^{\circ}, 79^{\circ}, 77^{\circ}\right.$, and $\left.63^{\circ}\right)$ are observed in the anion spectrum along with the $97^{+}$peak in the cation spectrum. Only the interfering peaks at $95^{\circ}, 79^{\circ}$, and $63^{\circ}$ may be identified with dursban. The spectra collected from the dandelion surface display weaker ion abundances than that collected from stainless steel; however, only the PMPA peaks $\left(179^{\circ}, 121^{\circ}, 95^{\circ}, 79^{\circ}, 77^{\circ}\right.$, and $\left.63^{\circ}\right)$ are observed. As with the stainless steel data, the only dursban peaks observed in the dandelion data are those listed above as interfering peaks. These data indicate that PMPA may be detected in the presence of dursban on either substrate. 
Fenthion/PMPA: In the stainless steel data, peaks due to PMPA are stronger than those due to fenthion. The PMPA peaks $\left(179^{-}, 121^{\circ}, 95^{\circ}, 79^{\circ}, 77^{\circ}\right.$, and $\left.63^{\circ}\right)$ are observed in the anion spectrum along with the $97^{+}$peak in the cation spectrum. The only fentbion peaks present in the stainless steel data are at $141^{-}$ and the interfering peaks at $95^{\circ}, 7^{\circ}, 63^{\circ}$. The dandelion data displays mainly the PMPA peaks which are weaker than those in the stainless steel data. The only identified fenthion peaks in the dandelion data are the interfering peaks at 95\%,79-, and 63-. These data indicate that PMPA may be detected in the presence of fenthion on either substrate.
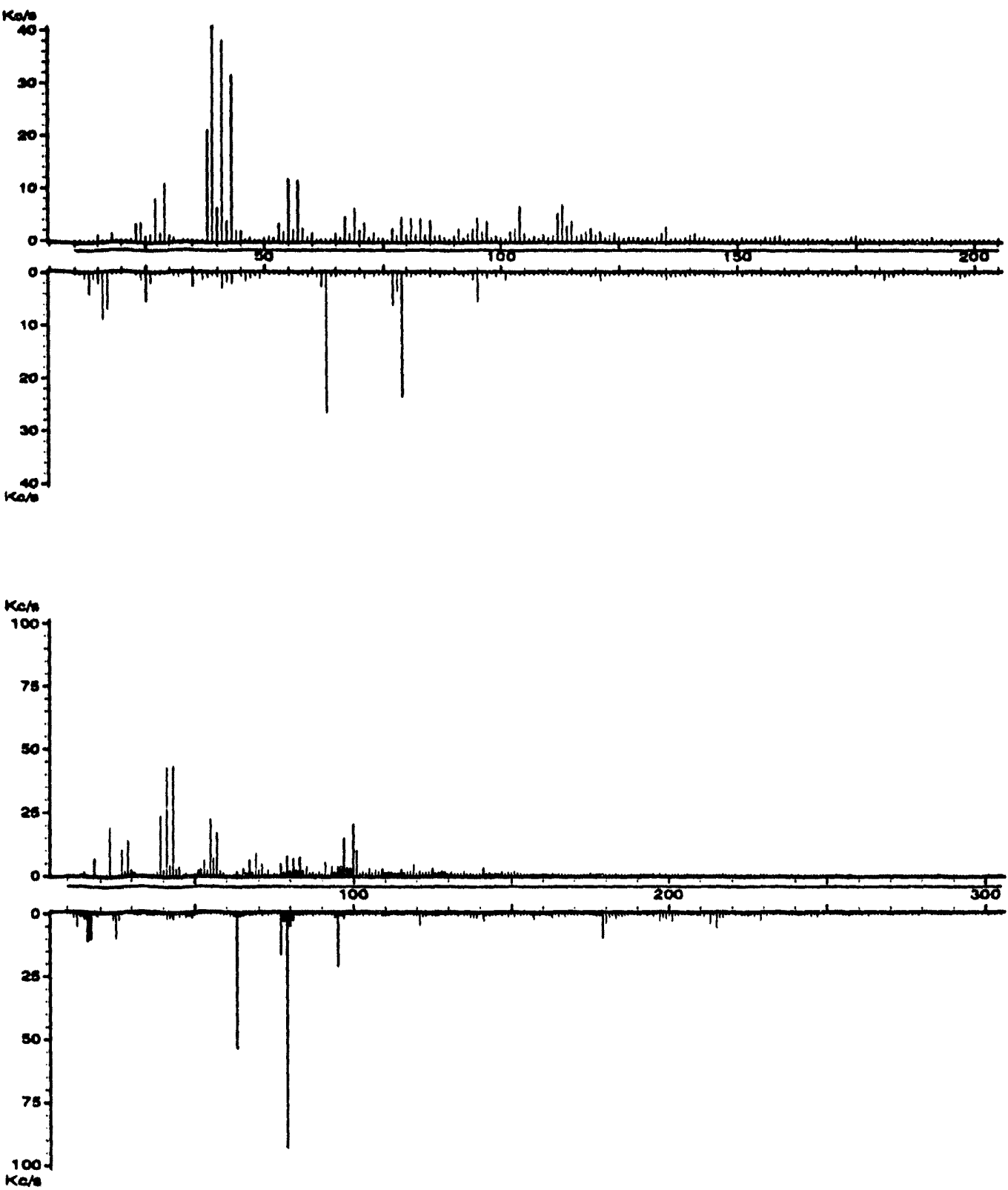

Figure 5. Fenthion and PMPA mixture on stainless steel (bottom) and dandelion (top). 
Mocap/PMPA: In the stainless steel data, peaks due to PMPA are stronger than those due to mocap. The PMPA peaks $\left(179^{-}, 121^{\circ}, 95^{\circ}, 79^{\circ}, 77^{\circ}\right.$, and $\left.63^{\circ}\right)$ are observed in the anion spectrum along with the $97^{+}$ peak in the cation spectrum. The only mocap peaks present in the stainless steel data are the interfering peaks at $95^{\circ}, 79^{\circ}$, and $63^{\circ}$. The dandelion data displays mainly the PMPA peaks which are weaker than those in the stainless steel data. The only identified mocap peaks in the dandelion data are the interfering peaks at $79^{\circ}$, and $63^{\circ}$. These data indicate that PMPA may be detected in the presence of mocap on either substrate.
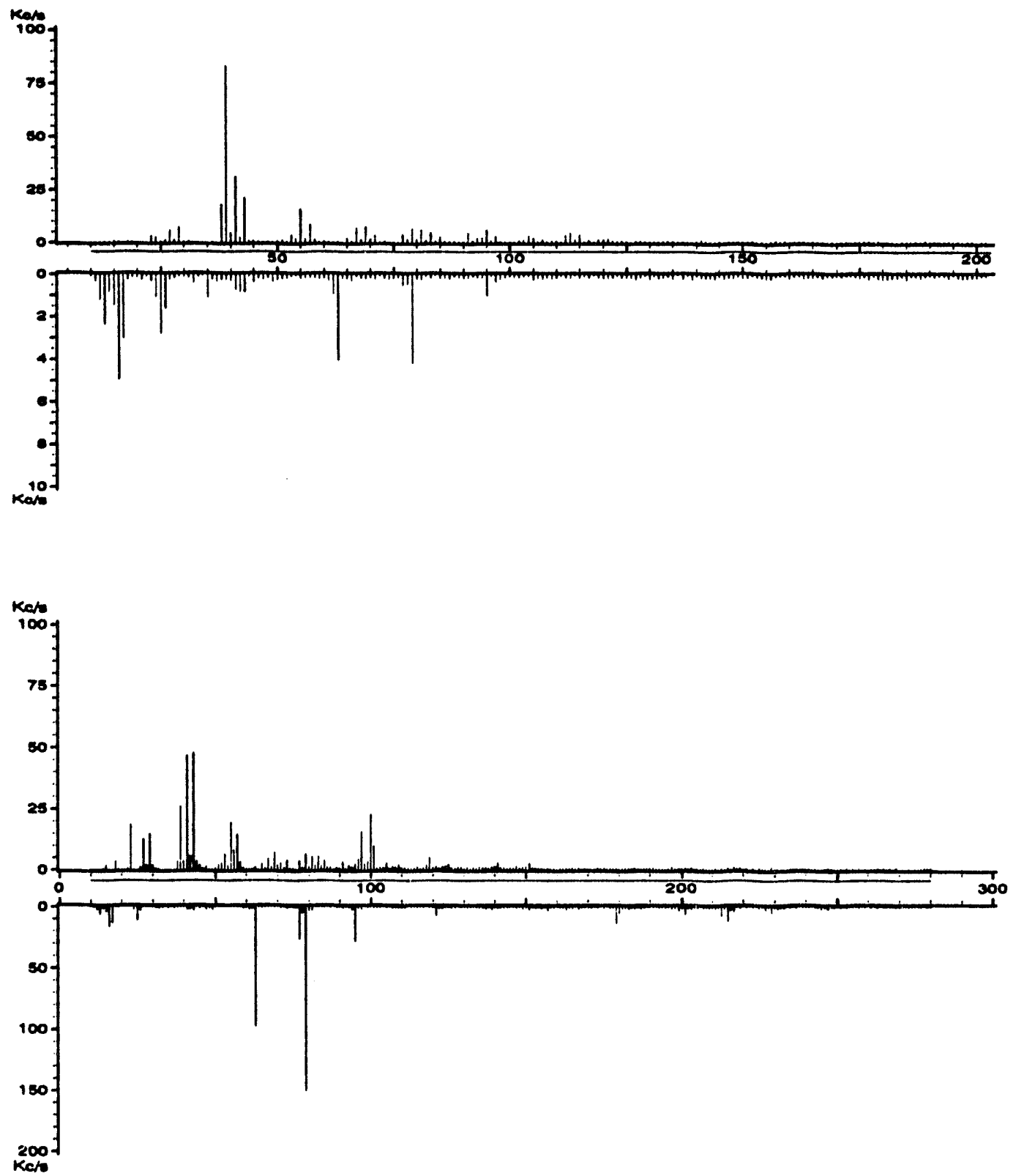

Figure 6. Mocap and PMPA mixture on stainless steel (bottom) and dandelion (top). 
DEF/PMPA: In the stainless steel data, peaks due to PMPA are stronger than those due to DEF. The PMPA peaks $\left(179^{\circ}, 121^{\circ}, 95^{\circ}, 79^{\circ}, 77^{\circ}\right.$, and $\left.63^{\circ}\right)$ are observed in the anion spectrum along with the $97^{+}$ peak in the cation spectrum. The only DEF peaks present in the stainless steel data are the interfering peaks at $79^{-}$and $63^{\circ}$. The dandelion data displays mainly the PMPA peaks which are weaker than those in the stainless steel data. The only identified DEF peaks in the dandelion data are the interfering peaks at $79^{-}$and $63^{\circ}$. These data indicate that PMPA may be detected in the presence of DEF on either substrate.
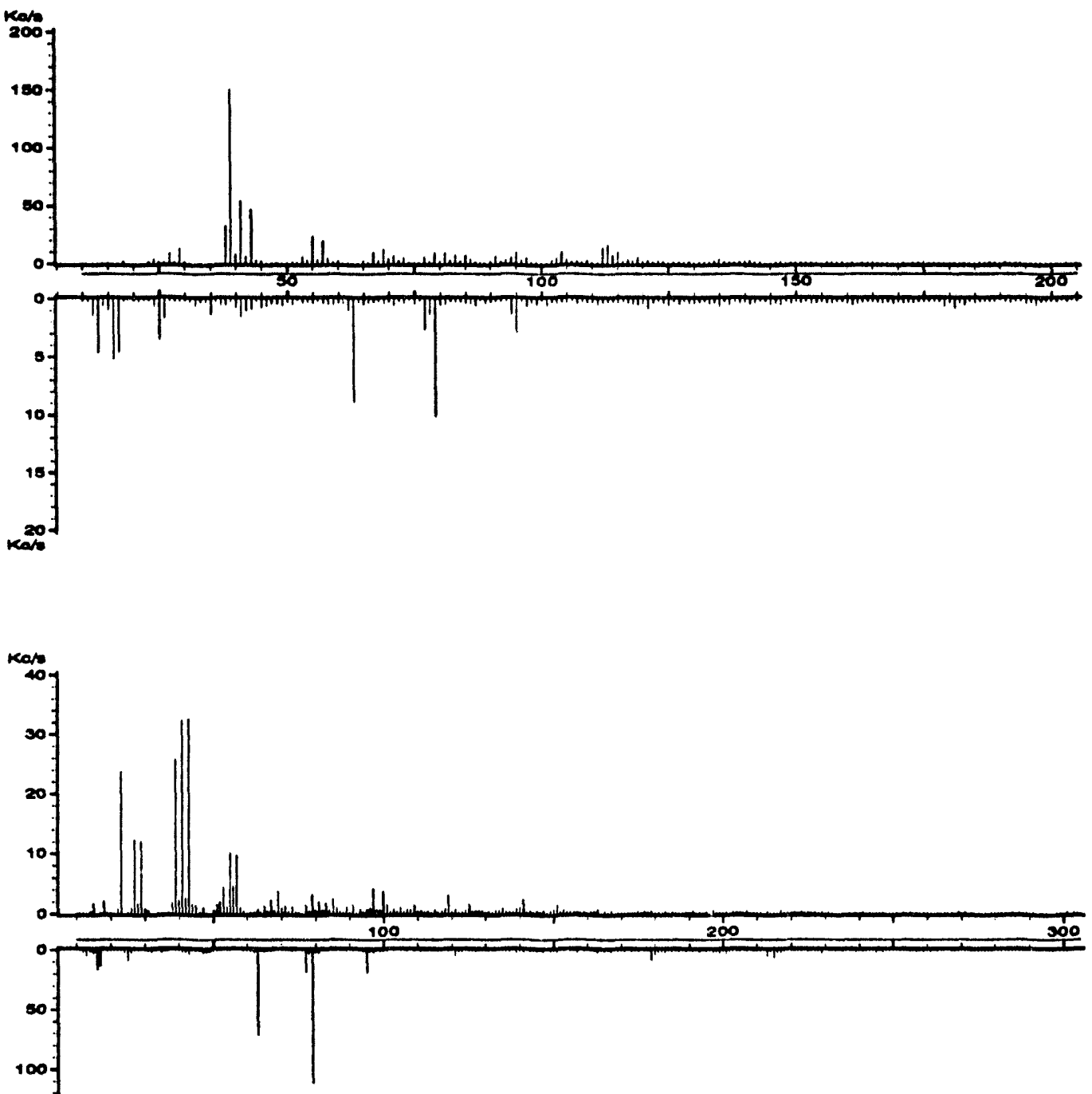

150.

Figure 7. DEF and PMPA mixture on stainless steel (bottom) and dande lion (top). 


\section{Conclusions}

The results of this study indicate that PMPA may be detected on stainless steel in the presence of all six pesticides/herbicides investigated. In the case of detection of PMPA from a dandelion surface, it was found only the abate may cause some difficulties in the PMPA detection. It is important to emphasize that these investigations were carried out with the pesticide/herbicide present at a factor of 8 to 10 times in excess of the PMPA. Possible explanations for the strong PMPA SIM spectra in the presence of the pesticides/herbicides investigated in these studies include: a) PMPA may have a higher ionization probability compared to the pesticide/herbicides on these types of substrates; b) less absorption of the PMPA into the substrate such that more is accessible for analysis, or c) the pesticides/herbicides preferentially adsorb to the substrate leaving the PMPA on the top layer where it is more readily sampled.

\section{Significance to Direction of Current Work}

The similarity in the $\mathrm{m} / \mathrm{z}$ values of fragments from the PMPA and from the sulfur/phosphorous containing compounds used in this study demonstrate how the detection of PMPA could be improved with MS/MS capabilities. In order to minimize the possibility of false positive detections it appears that the moleculas ion peak of the AMPA of interest must be detected, and to provide high confidence, an MS/MS scan of the molecular ion peak should be performed. Using this approach it appears that the AMPAs can be readily detected in the presence of environmental interferents on steel surfaces and vegetation surfaces. Thus, the MS/MS capability of the ion trap based MBSA must be fully integrated into the operating protocols of the system and should be optimized.

The need for detecting the molecular ion peak also points out the importance of the filtered noise system (see section I of this report) being incorporated in the MBSA instrument. In order to obtain the highest sensitivity it will be necessary to collect adequate quantities of molecular ions so that MS/MS can be utilized. The filtered noise system makes it possible to selectively collect only those jons of analytical interest in the ion trap.

The investigations of the single component systems demonstrate the advantage of collecting both the anion and cation spectra during each analyses. Many spectra collected for the pesticides/berbicides contained both anion and cation fragments which could be used in the detection of these analytes. While this improved efficiency, it has not yet been demonstrated as a firm requirement, and thus the tradeoffs involved in incorporating simultaneous detection of positive and negative ions in the ion trap MBSA must be further evaluated before a final decision is made.

The role that surface chemistry plays in the production of secondary ions must be further examined, for it appears to present opportunities to both improve detectability, and to enhance selectivity. Further studies should be conducted to elucidate the effects of surface chemistry. 


\section{Appendix III-A}

Individual mass spectra of the test compounds used in the interference study, cations on top, anions on bottom.

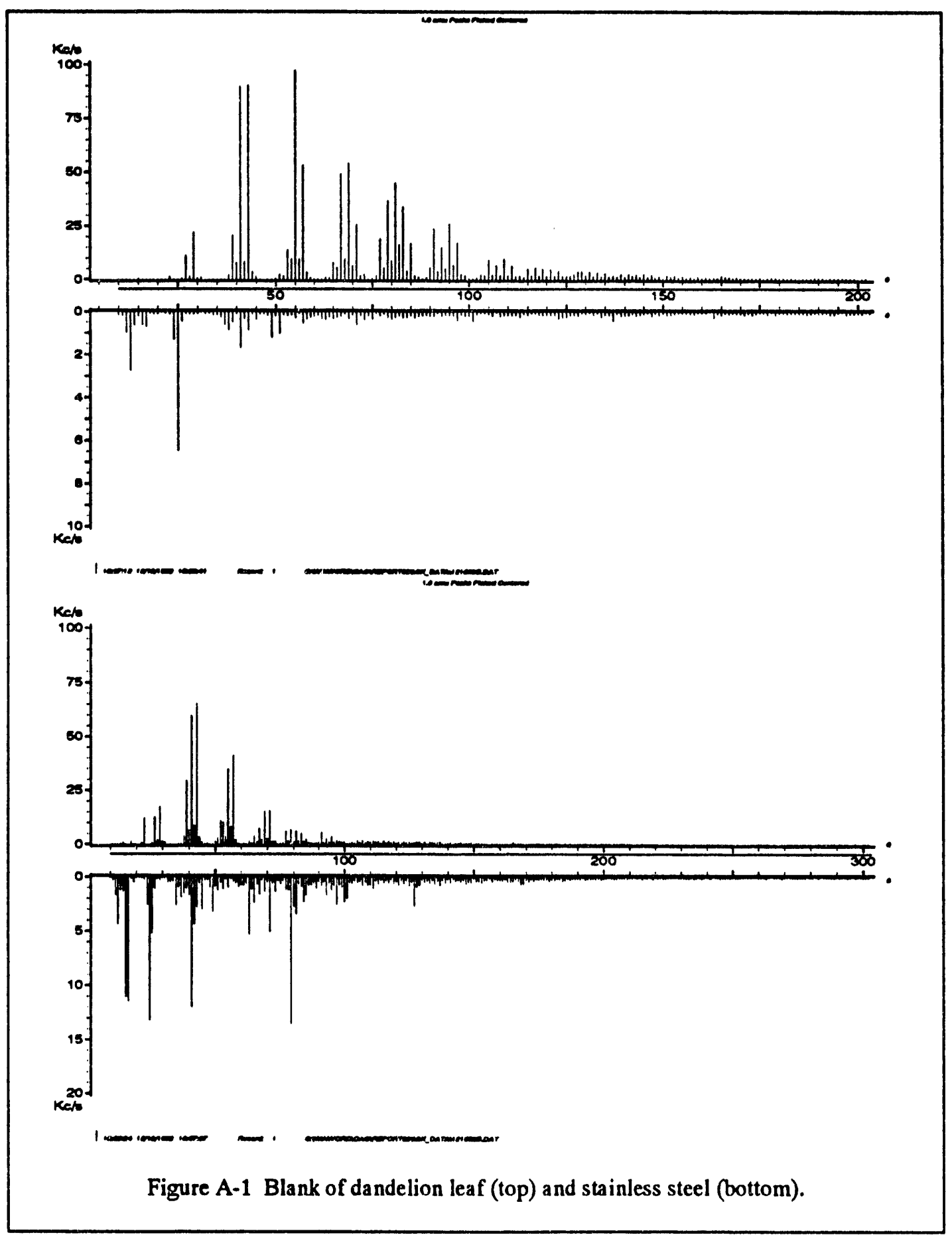




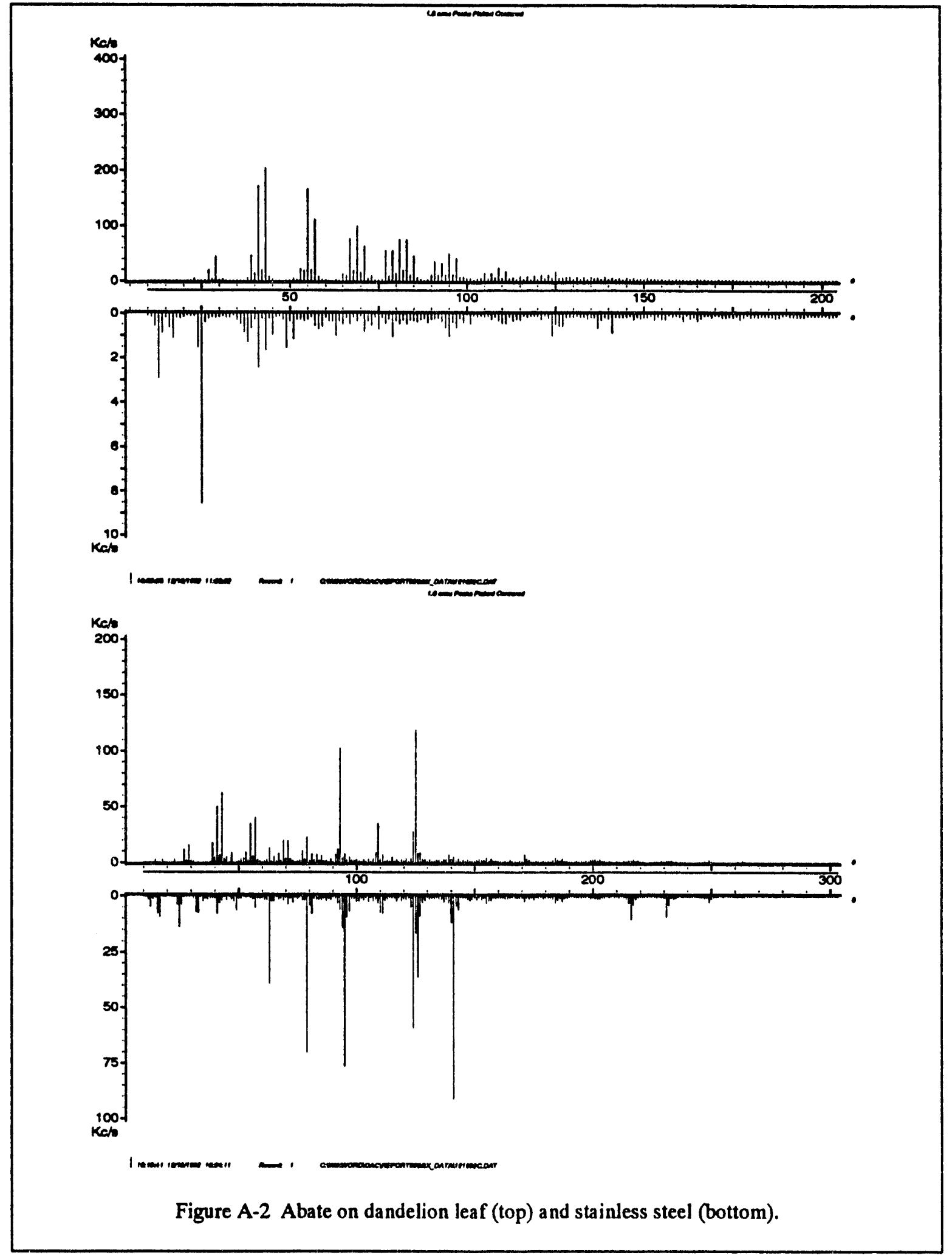




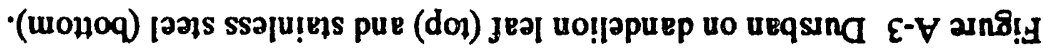
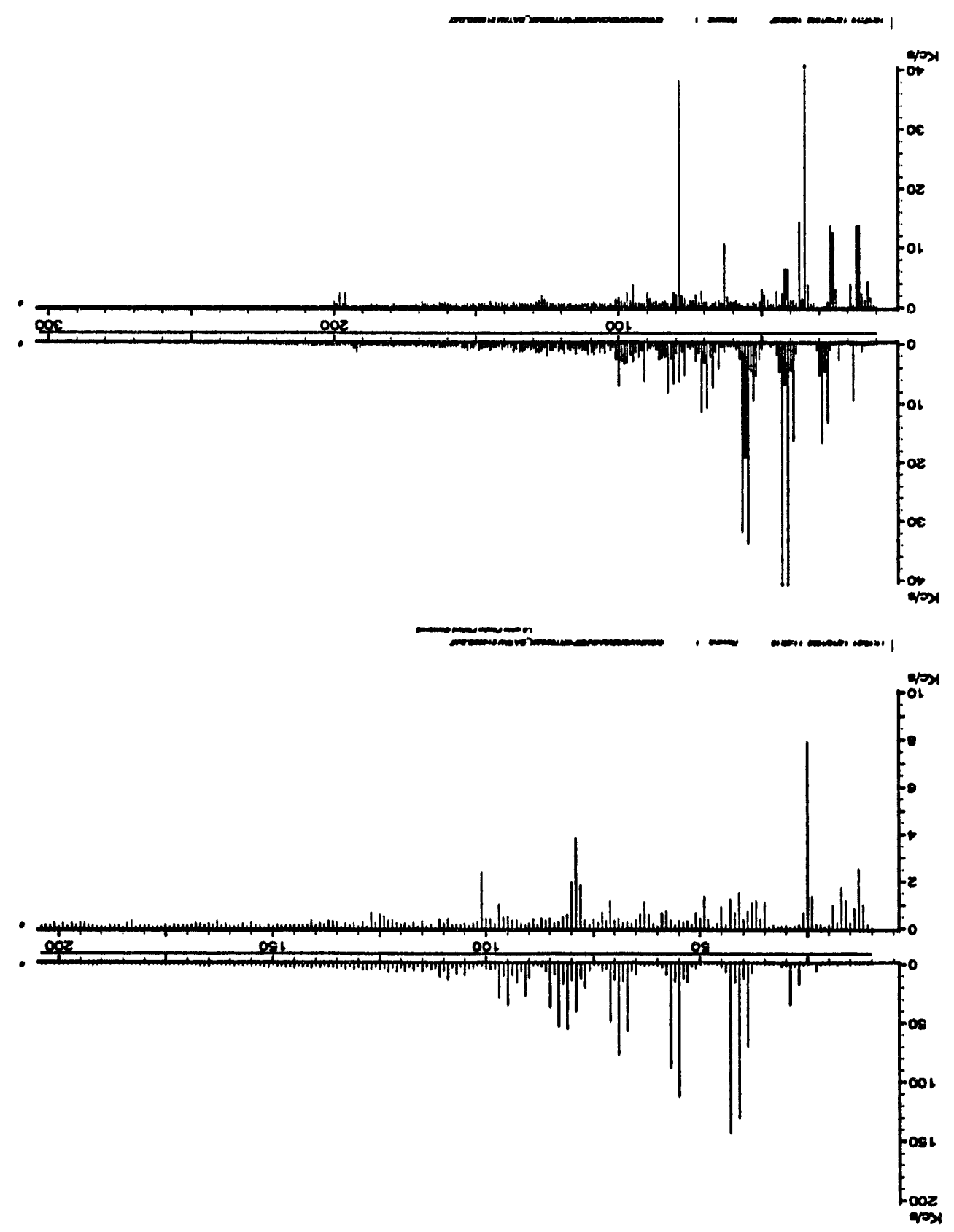


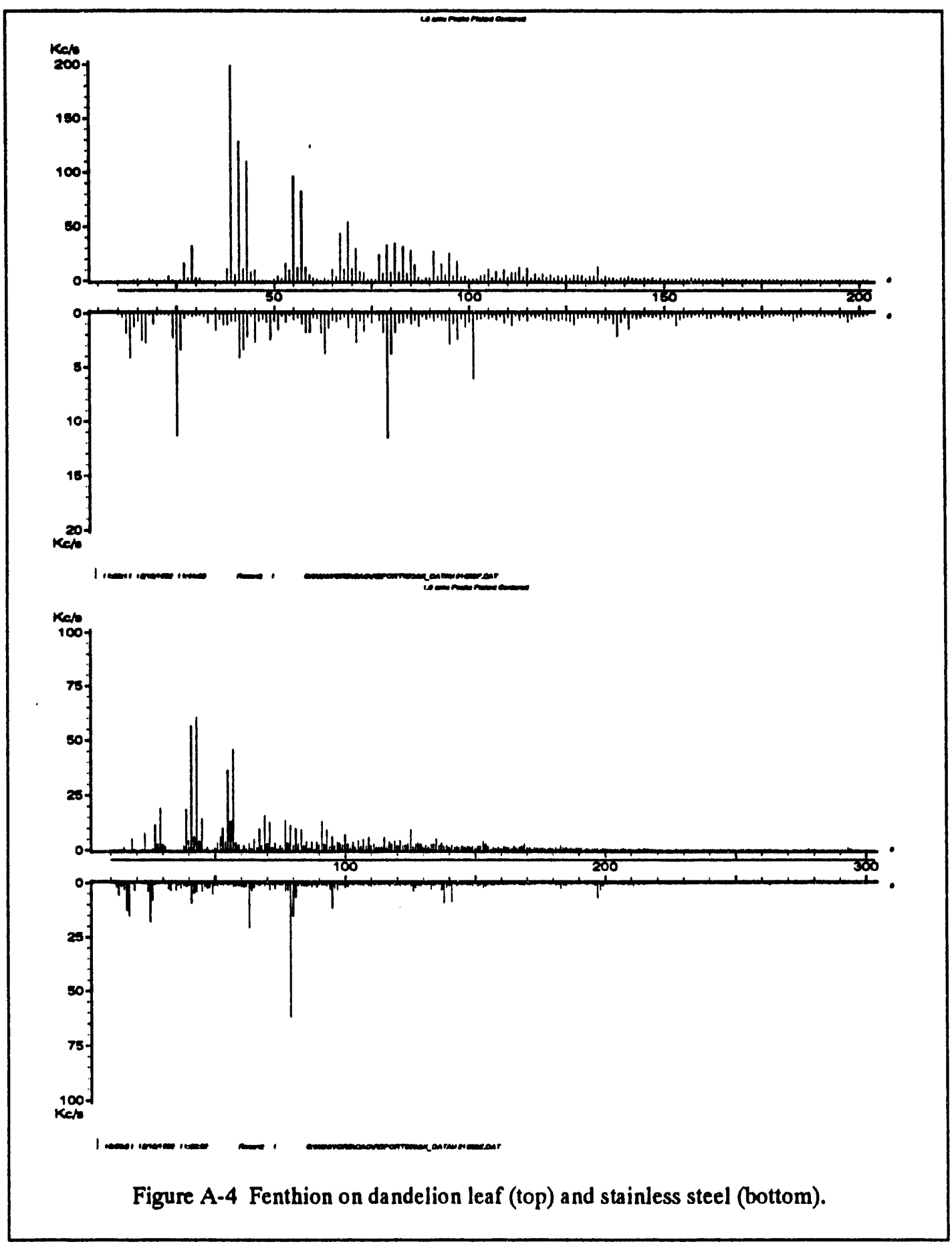




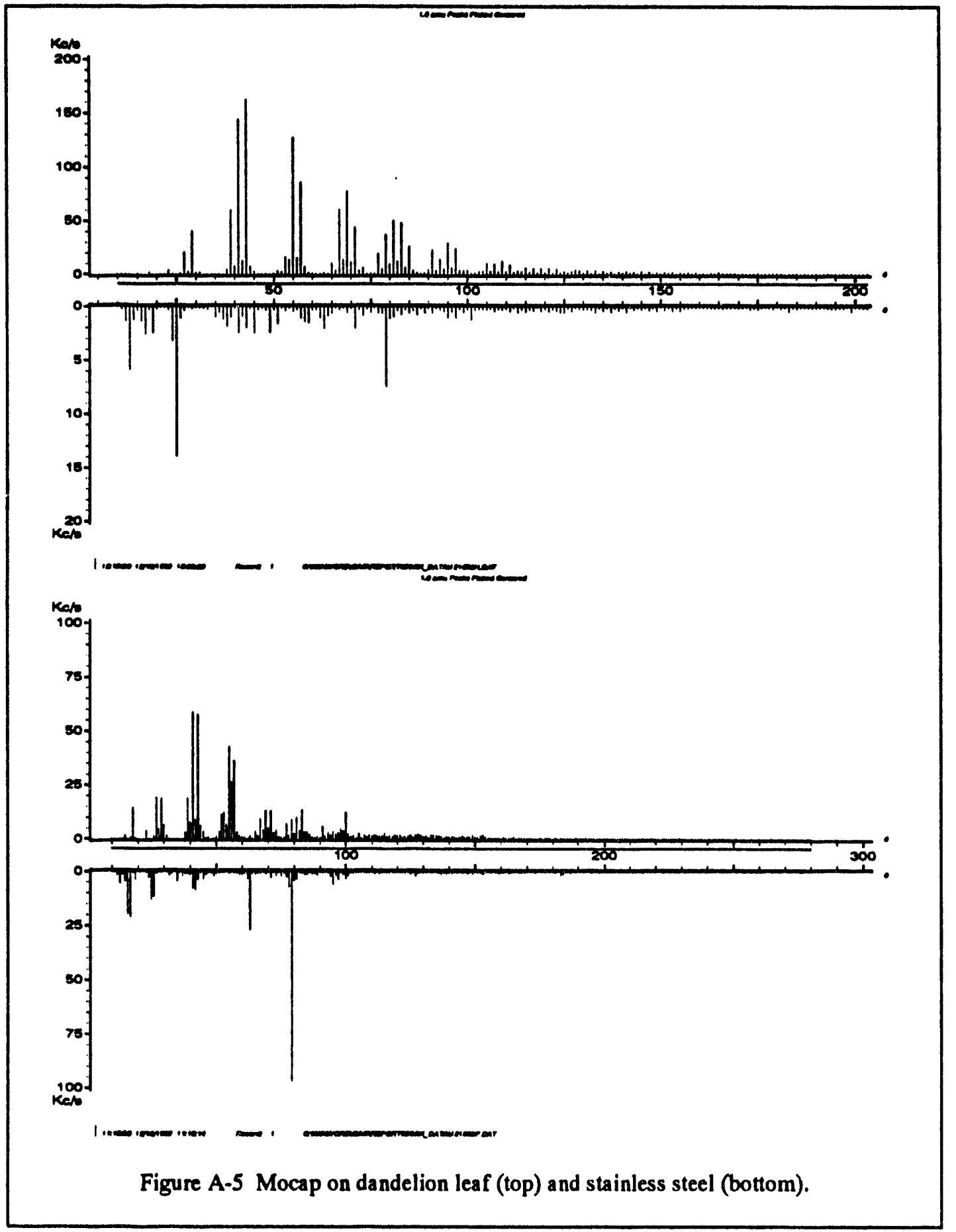




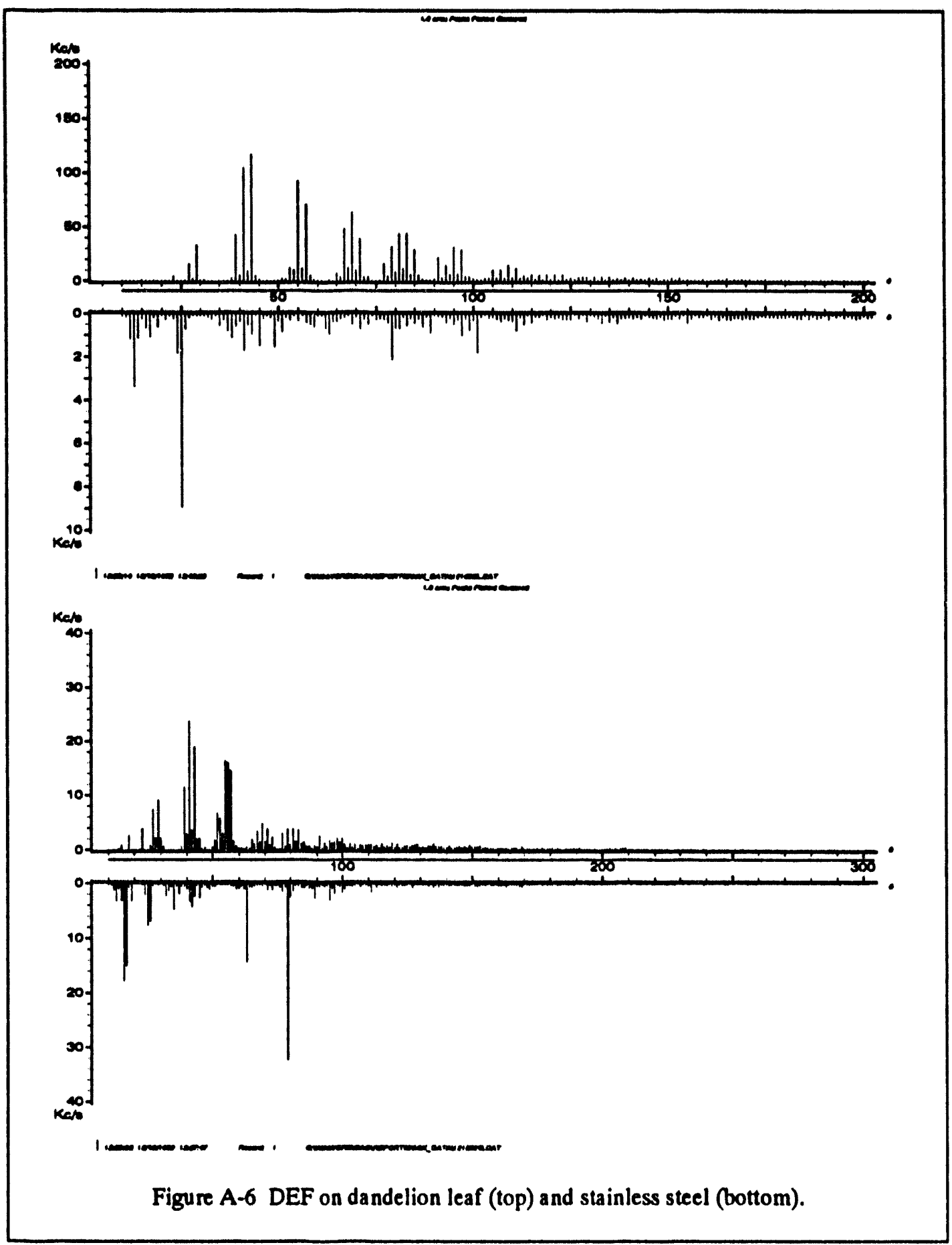




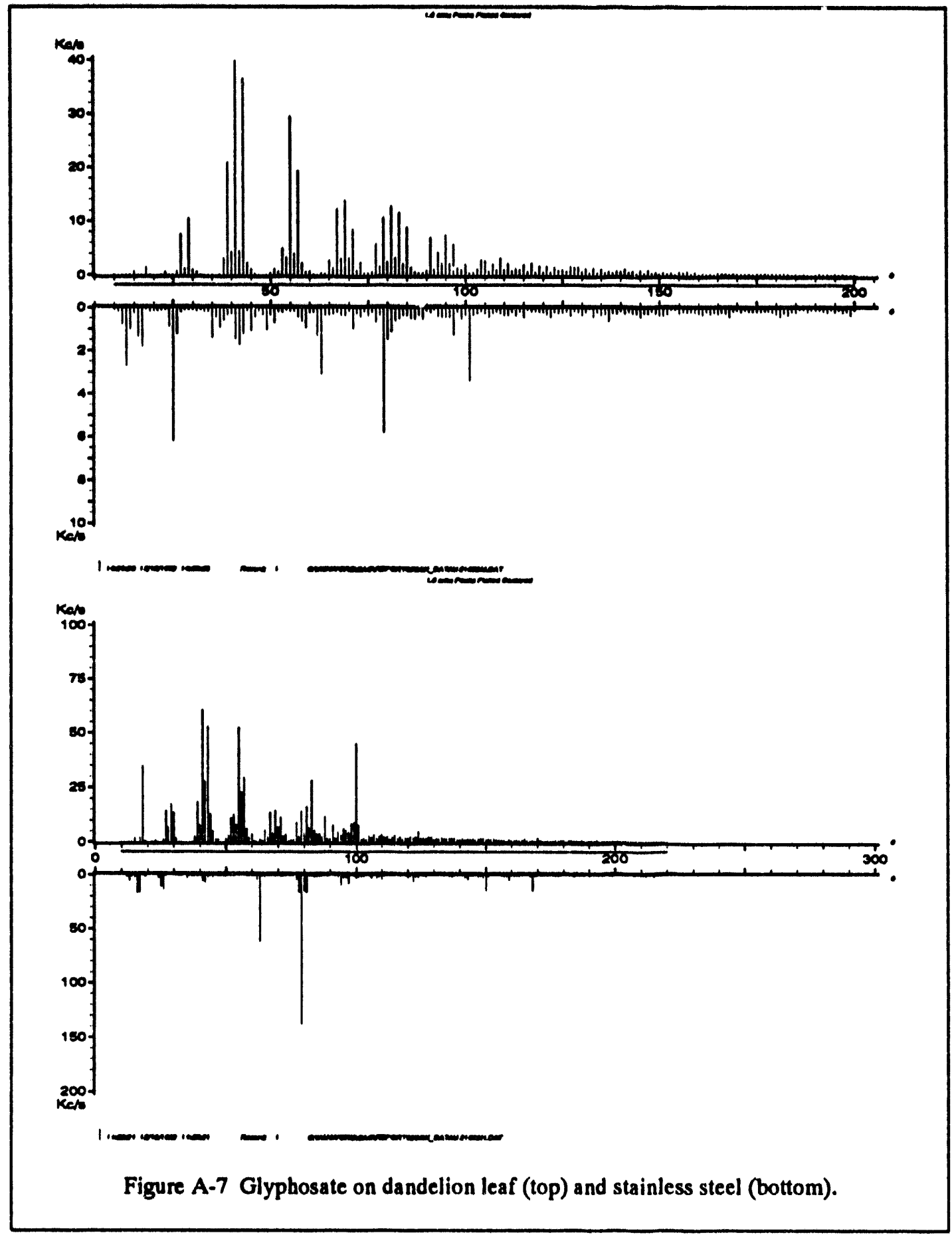




\title{
IV Sample Variability and Anecdotal Experiments
}

\author{
Effects of Sample Varlability on Detection of AMPAs on Vegotation
}

\section{Introduction}

The purpose of these studies was to investigate the secondary ion emission of alkylmethylphosphonic acids (AMPA) from a variety of leaf surfaces using molecular beam surface analysis (MBSA). In previous studies, surface analysis of AMPAs on vegetation surfaces using MBSA was investigated using leaves from dandelion and common bouse plants. Results from these studies suggested that the secondary ion mass spectra of the AMPAs was not dependent on the leaf surface. However, due to the minimal knowledge of the surface chemistry of these leaves and the lack of variety in these leaves, a more vigorous study was warmated. In the present study, isopropyl methylphosphunic acid (IMPA) was spiked on 10 different leaves and analyzed using MBSA. These leaves were chosen based on information published by Stevens and Baker ${ }^{1}$ conceming their surface cbemistry. IMPA was chosen as a representative AMPA.

\section{Experimental}

The quadrupole-based secondary ion mass spectrometer (SIMS) used in the previous vegetation studies was also used in the present work. The leaves used in this study were collected from the following plants: potato, pea, apple, strawberry, bean, corn, beet, rape, cotton, and lemon. Leaves from potato, pea, apple, strawberry, bean, and com were collected fresh (within 24 bours of the analysis) from a local garden. These plants were not treated with any type of agrochemical. The rape leaf was taken from a local field also within 24 hours of analysis. The cotton and lemon leaves were acquired from the Phoenix, Arizona area three days before analysis. Agrochemical treatment of the rape, cotton, and lemon plants is not known. A 100 ppm IMPA aqueous solution was used to spike the leaves.

Prior to analysis, three disks of $3.8 \mathrm{~mm}$ diameter were cut from the leaf with a bole boring tool. The leaf disks were attached to stainless steel planchettes using double-stick tape. After attaching the leaf disks to the planchettes, two leaf disks were spiked with 5 ul of the 100 ppm IMPA solution. One leaf disk was left blank for background analysis. The leaves were allowed to air dry for $\sim 2$ bours before analysis. The sample was then inserted into the instrument and analyzed at a pressure of $5 \times 10^{-7}$ torr. The approximate surface loading of these samples is $35 \mathrm{ng} / \mathrm{mm}^{2}$.

The data was acquired over the mass range of $m / 210$ to 150 . In each spectrum acquired, two scans were signal averaged. Only the anion spectra are discussed in this report; the cation spectra did not contain information useful in identifying IMPA. The spectra were normalized to the $m / 225$ peak $\left(\mathrm{C}_{2} \mathrm{H}^{-}\right)$to account for minor fluctuations in the primary beam intensity. This peak was chosen as it was reproducibly present on both the leaf blank and the IMPA spiked leaves.

\section{Results}

Blank Leaves. Chemical characterization has been reported by Stevens and Baker 1 on the epicuticular waxes of the leaf types used in these experiments. The epicuticular waxes make-up the outer surface of the leaf. In the Stevens and Baker study, the epicuticular waxes were extracted from the leaves using a wash containing a mixture of chloroform and diethyl ether. The extracts were analyzed using thin layer chromatography, gas chromatography, and gas chromatography/mass spectrometry. The surface areas of

1Stevens, P. J. G.; Baker, E. A.; Pestic. Sci., 1987, 19, 265-281. 
each leaf were also determined using an imaging system and were reported in terms of the wax deposited per unit area. The compositions of the waxes and the amount of wax deposited for each leaf type are listed in Table 1.

Table 1. Chemical composition and surface area of epicuticular waxes from various leaves 1

\begin{tabular}{|c|c|c|c|c|c|}
\hline Composition (\%) & Apple & Bean & Cotton & Lemon & Corn \\
\hline Hydrocarbon & 7.6 & 1.0 & 11.0 & 3.8 & 9.0 \\
\hline Alcohol & 50.2 & 15.8 & 83.4 & 96.2 & 70.2 \\
\hline Acetate & 18.9 & 81.6 & & & \\
\hline Ketone & 1.6 & & & & \\
\hline Triterpenoid & 20.5 & & & & \\
\hline Ester & 4.5 & & & & 17.4 \\
\hline Wax Deposit $\left(\mu \mathrm{g} / \mathrm{cm}^{2}\right)$ & 7.5 & 3.1 & 7.7 & 5.6 & 3.8 \\
\hline
\end{tabular}

\begin{tabular}{|c|c|c|c|c|c|}
\hline Composition (\%) & Pea & Potato & Rape & Strawberry & Beet \\
\hline Hydrocasbon & 9.8 & 45.7 & 42.6 & 15.8 & 2.9 \\
\hline Alcohol & 76.7 & 50.8 & 18.2 & 6.2 & 87.7 \\
\hline Acetate & & & & & \\
\hline Ketone & & & 35.8 & & \\
\hline Triterpenoid & & 0.7 & & 48.4 & \\
\hline Ester & 12.6 & & 3.2 & 24.2 & 1.1 \\
\hline Wax Deposit $\left(\mu \mathrm{g} / \mathrm{cm}^{2}\right)$ & 5.3 & 2.0 & 11.7 & 17.8 & 3.8 \\
\hline
\end{tabular}

The information shown in Table 1 indicates that the chemical compositions of the leaves examined in this study bave varied surface chemistries. Background SIM spectra were acquired of the various leaves used in this study with no IMPA present. An example of SIM spectra from two leaf surfaces is shown in Figure 1. The fragmentation was not interpreted in detail in these spectra; however, differences are observed in comparing these spectra which is consistent with the information published by Stevens and Baker. The remainder of the background SIM spectra are shown in the Appendix. 


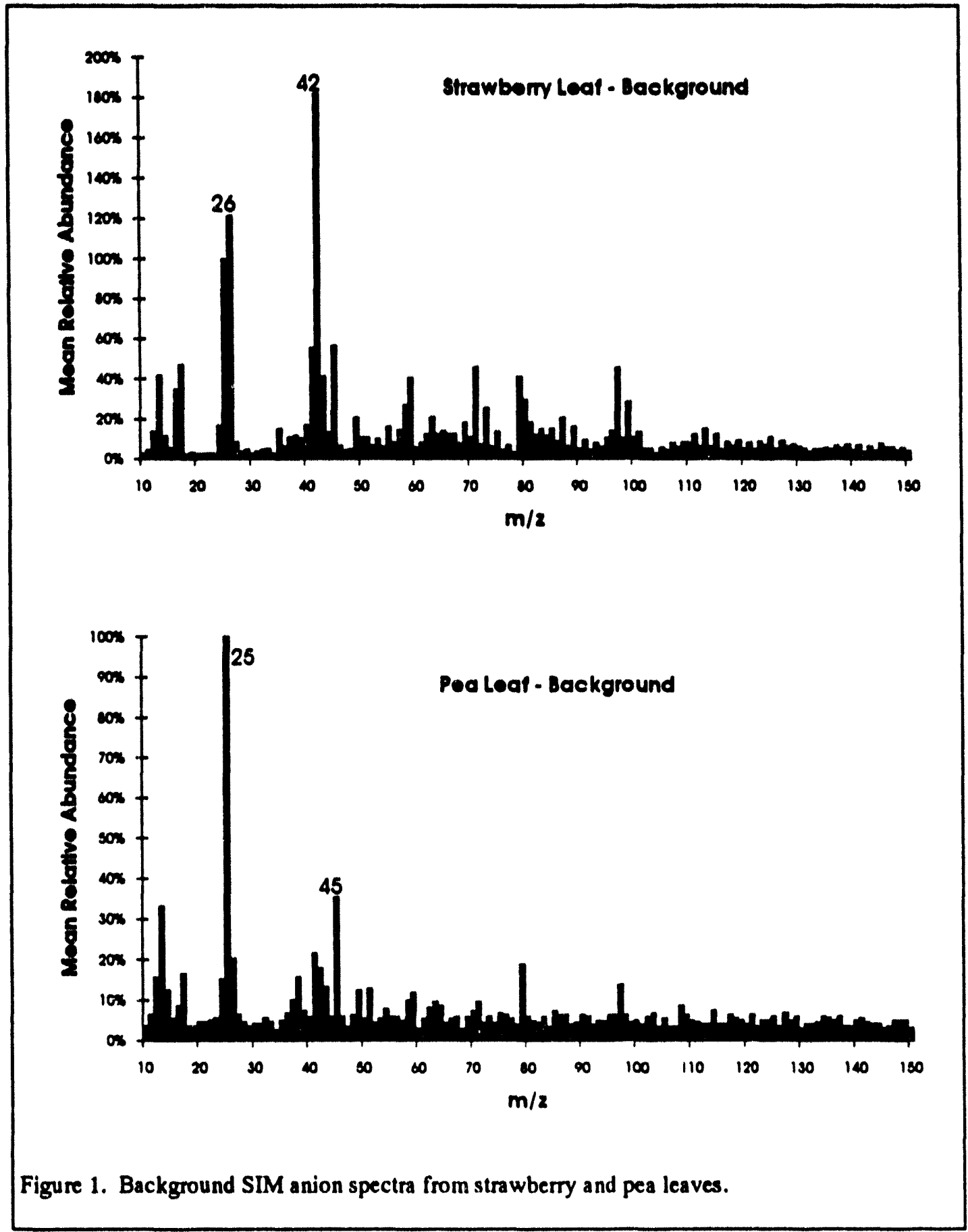


<smiles>CC(C)OP(C)(=O)[O-]</smiles><smiles>CP(=O)([O-])O</smiles>

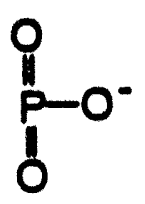

79<smiles>C[Po](C)=O</smiles>

77
0
1
0

63

Figure 2. Characteristic Anion Fragments of IMPA

The results from the MBSA study indicate that IMPA is readily detected on a variety of leaf surfaces. In Figure 3, examples of SIM spectra from IMPA on strawberry and pea leaves are shown. Although the blank spectn of these leaves, as shown in Figure 1, are dissimilar, the peaks characteristic of IMPA are easily observed on these two leaf surfaces. The remainder of the spectra from the other leaf types is presented in Appendix IV-A.

In Table 2, an overview of the results from all the leaf surfaces is given. The only leaf type in which the molecular ion, [IMPA-H]', is not present is on the apple leaf. Analysis of the apple leaf surface for IMPA was repeated on a different apple leaf to verify the results. The molecular ion peak is necessary in order to selectively detect for IMPA. Comparing the surface chemistry of the apple leaf to the other leaf types, it is more varied in composition with a mix of alcobols $(-50 \%)$, acetates $(-20 \%)$, triterpenoids $(-20 \%)$, hydrocarbon ( $-8 \%)$, and the remainder ketones and esters. The other leaf surfaces tend to bave two to three components with one of the components dominating the mixture. Additionally, when the IMPA solution was applied to the apple leaf, it did not wet the surface as well as the other leaves. Possible explanations for the absence of the [IMPA-H] in the SIM anion spectrum on the apple leaf include: a) the IMPA molecule may be strongly adsorbed to the leaf surface making it difficult to sputter into the gas phase as the molecular species, b) the IMPA molecule may absorb into the leaf making it inaccessible for MBSA detection.

IMPA Spiked Leaves. From previous MBSA work of IMPA on dandelion and house plant leaves, characteristic fragment ions of IMPA have been identified from the SIM spectrum and are shown in Figure 2. These peaks serve as the criteria for detectability of IMPA on the various leaf surfaces investigated in the present work. A signal to noise $>3$ was required to deem the peak significant. 


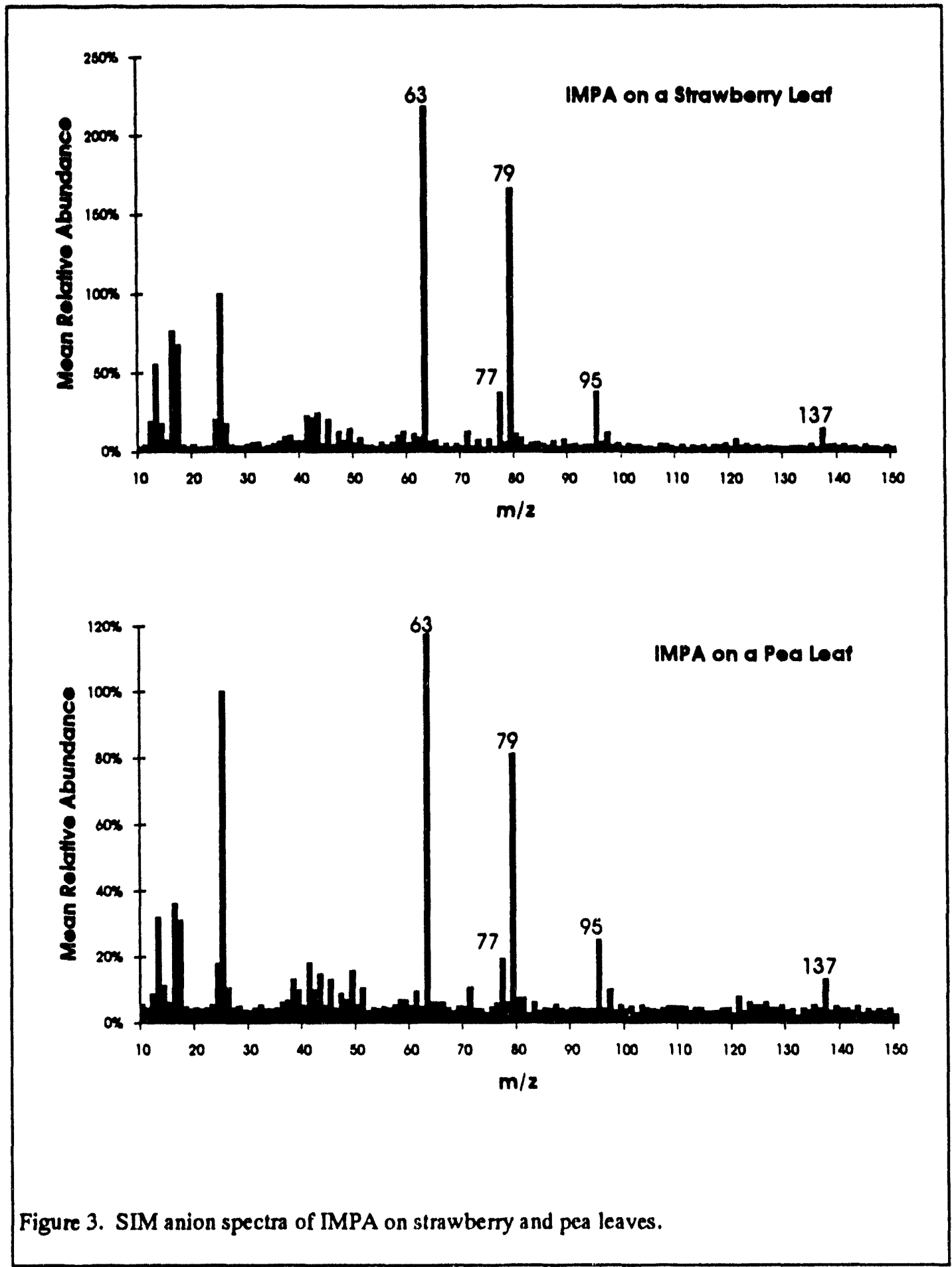


Table 2. Summary of detection of IMPA on various leaves

\begin{tabular}{|c|c|c|}
\hline Type of Leaf & [MMPA-H]* detected & $m / z 95,79,77,63$ detected \\
\hline potato & yes & yes \\
\hline pea & yes & yes \\
\hline apple & no & yes \\
\hline strawberry & yes & yes \\
\hline bean & yes & yes \\
\hline corn & yes & yes \\
\hline beet & yes & yes \\
\hline rape & yes & yes \\
\hline cotton & yes & yes \\
\hline lemon & yes & yes \\
\hline
\end{tabular}

\section{Conclusions and Significance to Direction of Current Work}

Detection of IMPA on a variety of leaf surfaces was investigated to determine possible effects of varying surface chemistry on detectability. Characteristic anion fragments of the IMPA molecule including the molecular ion, [IMPA-H]', were observed in the SIM spectra from all the leaf surfaces with the exception of the apple leaf. Chemical analysis by Stevens and Baker of the epicuticular waxes from leaf types used in these experiments showed that the composition of these waxes varied widely from containing mainly hydrocarbons to mainly alcohols. The epicuticular waxes make-up the outer surface of the leaves. For all but one leaf type investigated, the surface chemistries of the leaves did not impact the SIM anion spectra of IMPA. Based on these results, detection of AMPAs on most leaf types is readily accomplished using MBSA.

It is clear that while sample variability overall seemed to bave little effect on the ability of MBSA to detect the AMPA, for one case, the apple leaf, the molecular ion was not detected. Since it appears (see previous sections of this report) that detection of the molecular ion will be critical for providing high certainty of a positive detection (that is, preventing false positives), we feel it is important to understand what factor(s) of the apple leaf surface are inhibiting production of the molecular ion. Thus future work should address this issue, possible looking at the other AMPAs, varying concentration, and perhaps attempting further characterization of the chemical and physical characteristics of the apple leaf as compared to the other leaves examined. 


\section{Anecdotal Experiments Illustrating Unique Capabilities of MBSA}

\section{Detection of Mustard Decomposition Products on Concrete, Pocks and Soll}

Detection of a decomposition product of mustard, thiodiglycol sulfoxide (TDGO), on concrete, rock and soil samples by molecular beam surface analysis was investigated to determine whether these sample types are amenable for MBSA investigation. Historically the detection of CW degradation products on concrete has been very difficult, requiring involved chemical extraction techniques.

\section{Concrete}

In the this study, a piece of concrete from a weathered sidewalk was used. Background MBSA was accomplished by analysis of a concrete chip "as is". The background spectrum from the an untreated concrete chip is shown in Figure 4. This spectrum is typical of surfaces exposed to organic materials which are ubiquitous to the environment.

Investigation of TDGO detectability by MBSA on concrete was accomplished by exposing concrete chips to aqueous solutions of TDGO. The concrete chips were placed in a small beaker of the TDGO solution for approximately 4 hours, then removed to evaporate the solvent. TDGO was detected on the concrete at $10,000 \mathrm{ppm}$ as evidenced by a peak at $m / z 139$ shown in the cation SIM spectrum in Figure 4. The peak
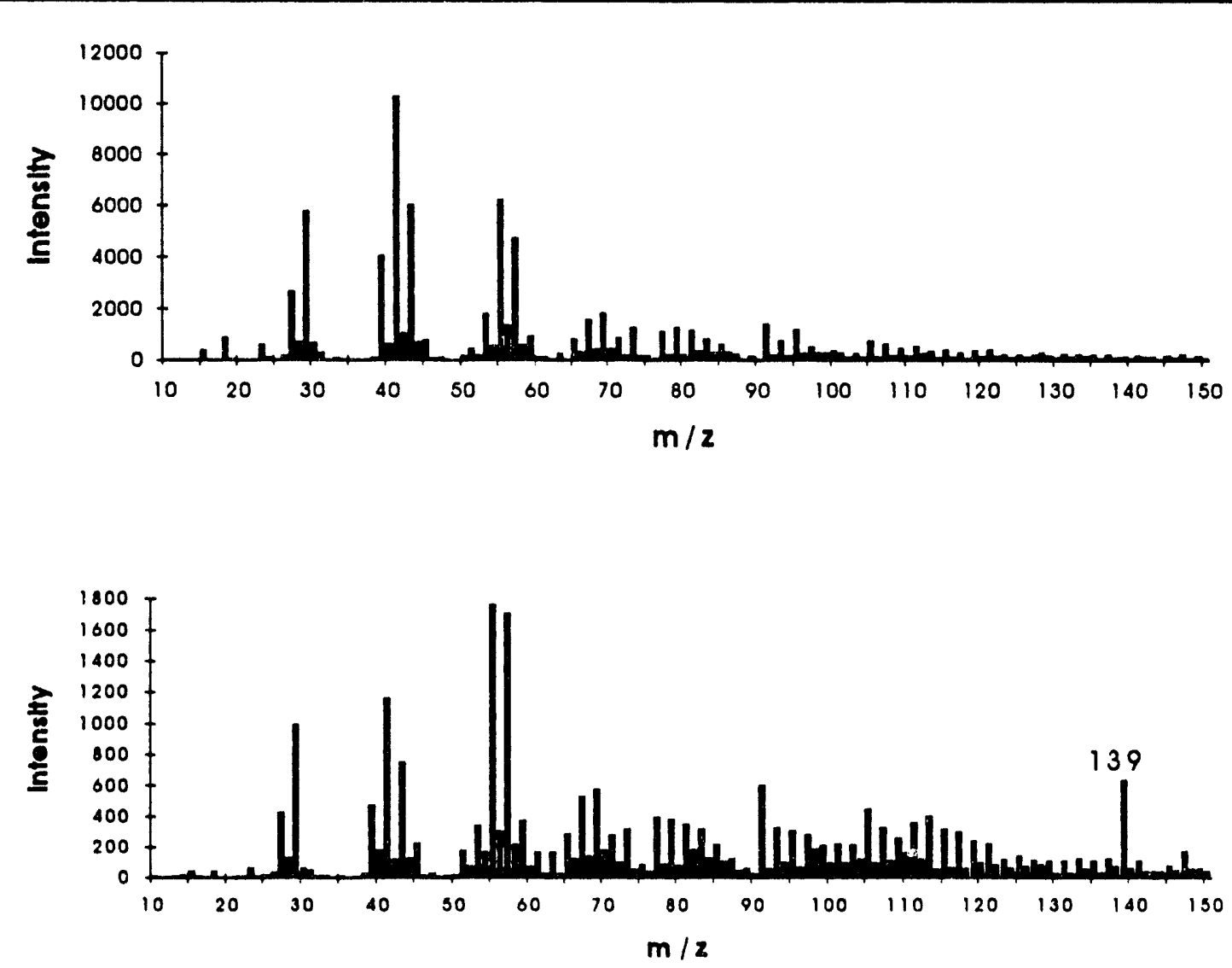

Figure 4. Background cation SIM spectrum of concrete (top) and of TDGO on concrete (bottom). 
at $m / 2139$ is attributed to [TDGO+H] ${ }^{+}$; the structure is shown in Figure 5.

\section{Rock and Soil Study}

Quartz-like rocks and top soil from an area near Edison, New Jersey were used to investigate the detectability of TDGO by MBSA on rock and soil surfaces. The rocks used in this study were small pebbles approximately $5 \mathrm{~mm}$ in diameter. Both pebbles and small quantities<smiles>OCC[SH](O)CCO</smiles>

Figure 5. [TDGO+H] ${ }^{+}$structure. $(-0.05 \mathrm{gm})$ of soil were exposed to TDGO solutions similar to the concrete chips. Before analysis, the pebbles were attached to a sample planchette with double stick tape. The soil was samples by pressing double stick tape into the soil sample. TDGO was detected on both the pebble and the soil at exposure to a 10,000 ppm solution as evidenced by a peak at $m / z 139$ shown in the cation SIM spectrum in Figures 6 and 7.
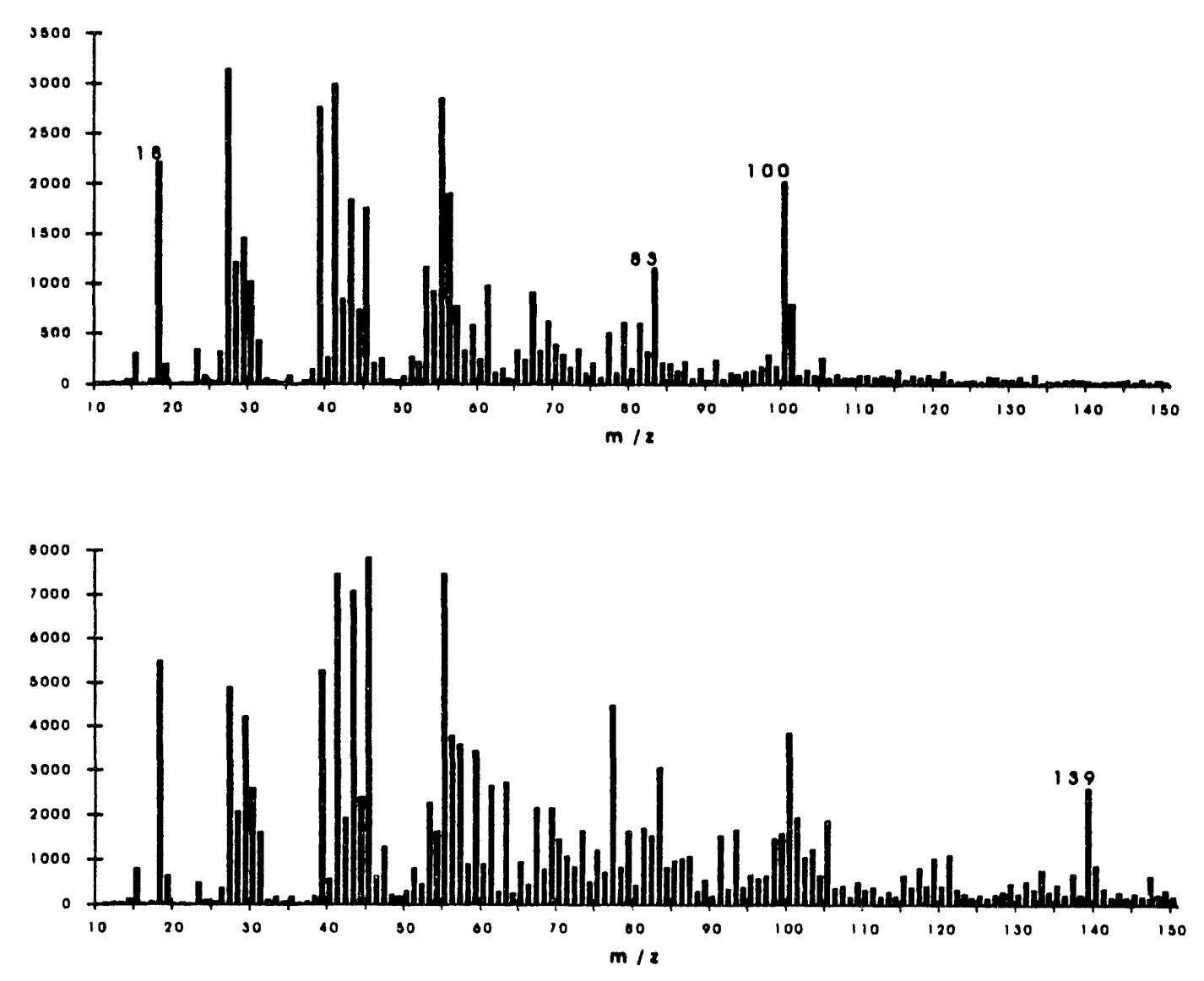

Figure 6. Cation spectrum of weathered rock (top) and rock exposed to TDGO (bottom).

It is interesting to note that in the background SIM spectra of both the rock and soil (Figures 6 and 7), peaks at $m / z \quad 100,83$, and 18 standout from the typical hydrocarbon background noted in Figure 4, the background spectrum associated with concrete. These peaks are attributed to cyclohexylamine, an indoor contaminant identified in our laboratory. A brief discussion of these findings is given later in this section. 


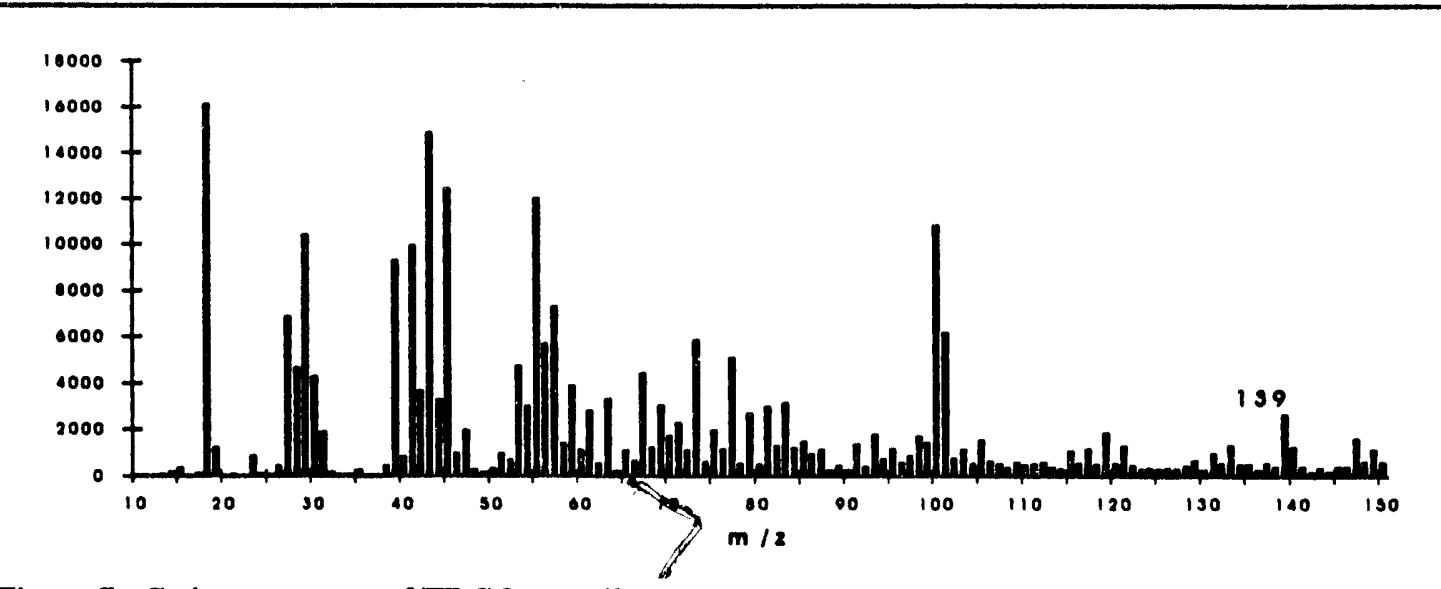

Figure 7. Cation spectrum of TDGO on soil.

\section{Detection of Airborne Contaminants Using Selective Surfaces}

In the investigation of background SIM spectra of a variety of sample types, it was found that cyclohexylamine (CHA) is an indoor contaminant in our laboratory. CHA is used to inhibit scale formation and pipe deterioration in the lab boiler, steam from which is used to humidify our facility. The mean concentration of CHA in the laboratory air was calculated to be $\sim 200 \mathrm{ppb}$, assuming that all of the CHA inserted into the boiler is evaporated into the laboratory air. This probably represents the maximum concentration. Recently we attempted to measure the concentration by taking an air sample, however, the concentration was below detection limits. Even at these extremely low levels, a sample brought into the laboratory acquired adequate CHA for detection with MBSA after $\sim 1$ hour exposure. Ions derived from $\mathrm{CHA}$ are observed on the sample surface at $m / 2100,[\mathrm{M}+\mathrm{H}]^{+} ; 83, \mathrm{C}_{6} \mathrm{H}_{11}{ }^{+}$, and $18, \mathrm{NH}_{4}^{+}$. An example of typical spectra of CHA observed on rock and soil surfaces can be seen in Figure 6 . These ion assignments were verified by treating the surfaces with $\mathrm{D}_{2} \mathrm{O}$, whereupon $\mathrm{m} / z 100$ shifted to 103 , and $m / z$ 18 shifted to 21, thus indicating three exchangeable protons. Surfaces on which CHA has been observed include steel, rocks, and soils. These findings suggest the application of MBSA to gas-phase monitoring by analyzing the surfaces of materials exposed to laboratory air, factory effluents, etc.

\section{Application to Nuclear non-Proliferation}

In a program sponsored by the Department of Energy Office of Waste Management we have investigated the application of MBSA to detection of compounds used in the nuclear weapons production cycle. A primary example is the compound tributyl phosphate (TBP), used as a complexing agent in the processing of uranium and plutonium. We found that TBP could be detected when the samples were exposed to concentrations of TBP in water at levels as low as $10 \mathrm{ppm}$. 


\section{Appendix IV-A}

Individual mass spectra of blank plant leaves and plant leaves spiked with IMPA.

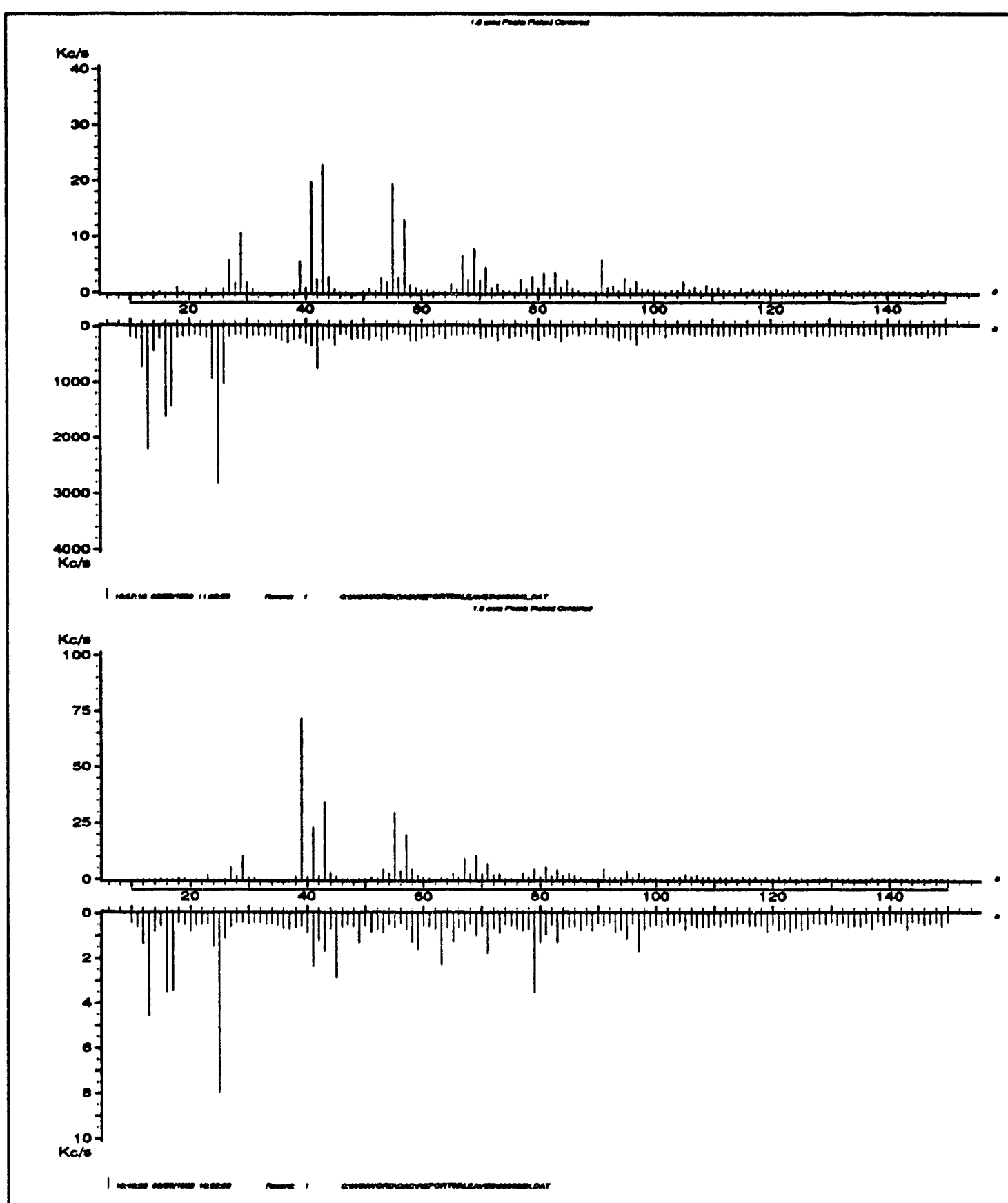

Figure A-1. Secondary ion mass spectrum of blank apple leaf (top) and leaf spiked with IMPA(bottom). 


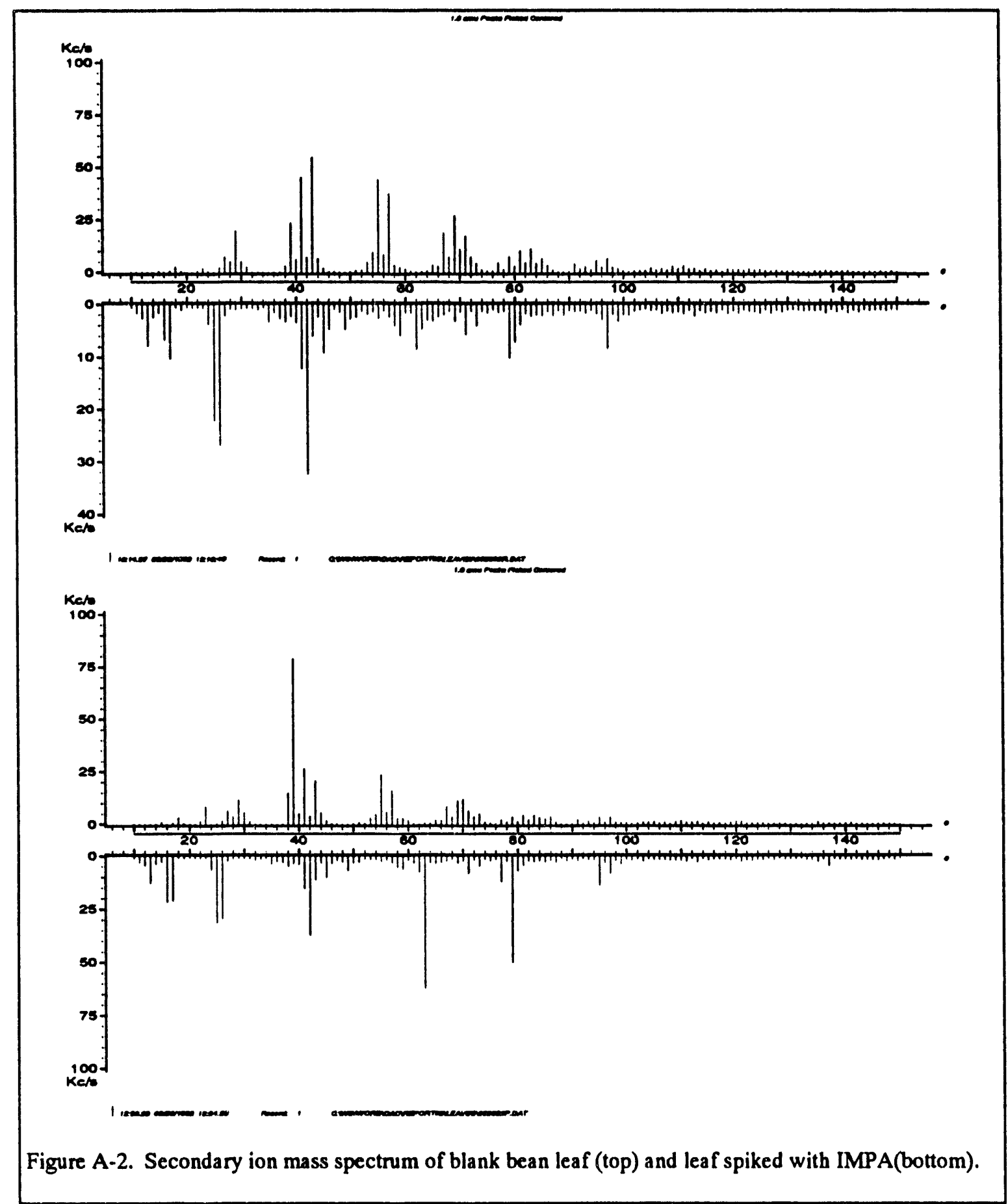




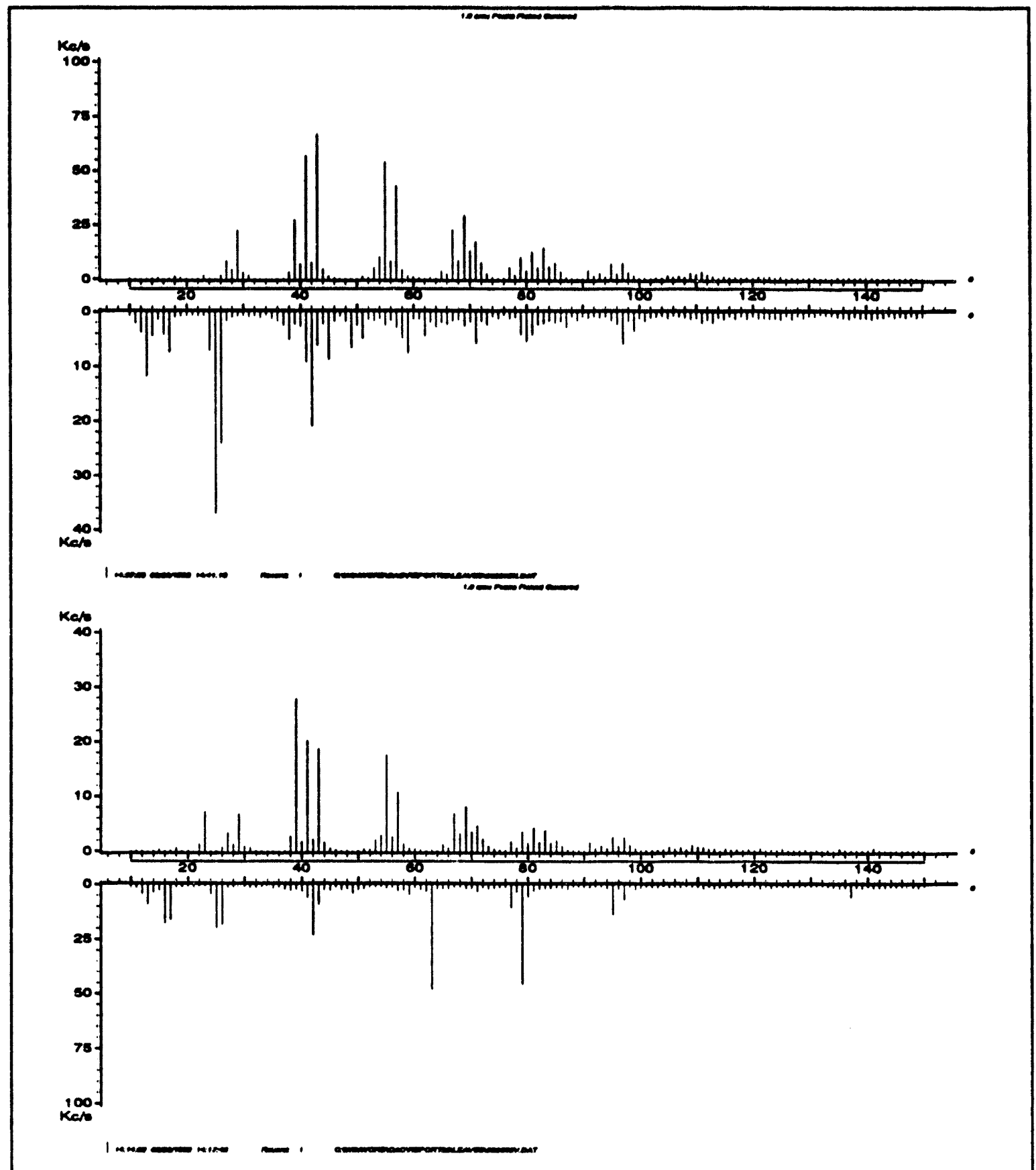

Figure A-3. Secondary ion mass spectrum of blank beet leaf (top) and leaf spiked with IMPA(bottom). 


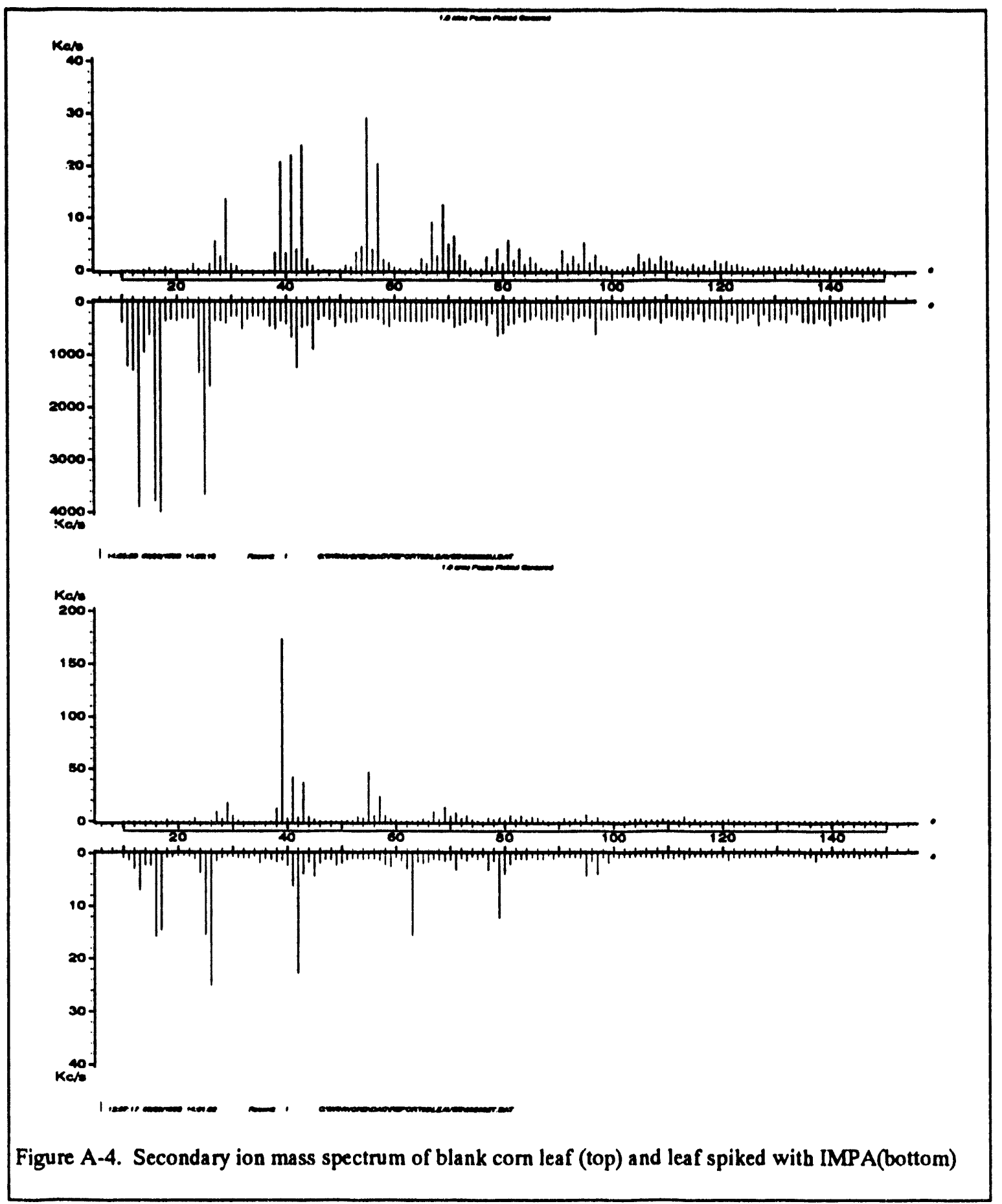




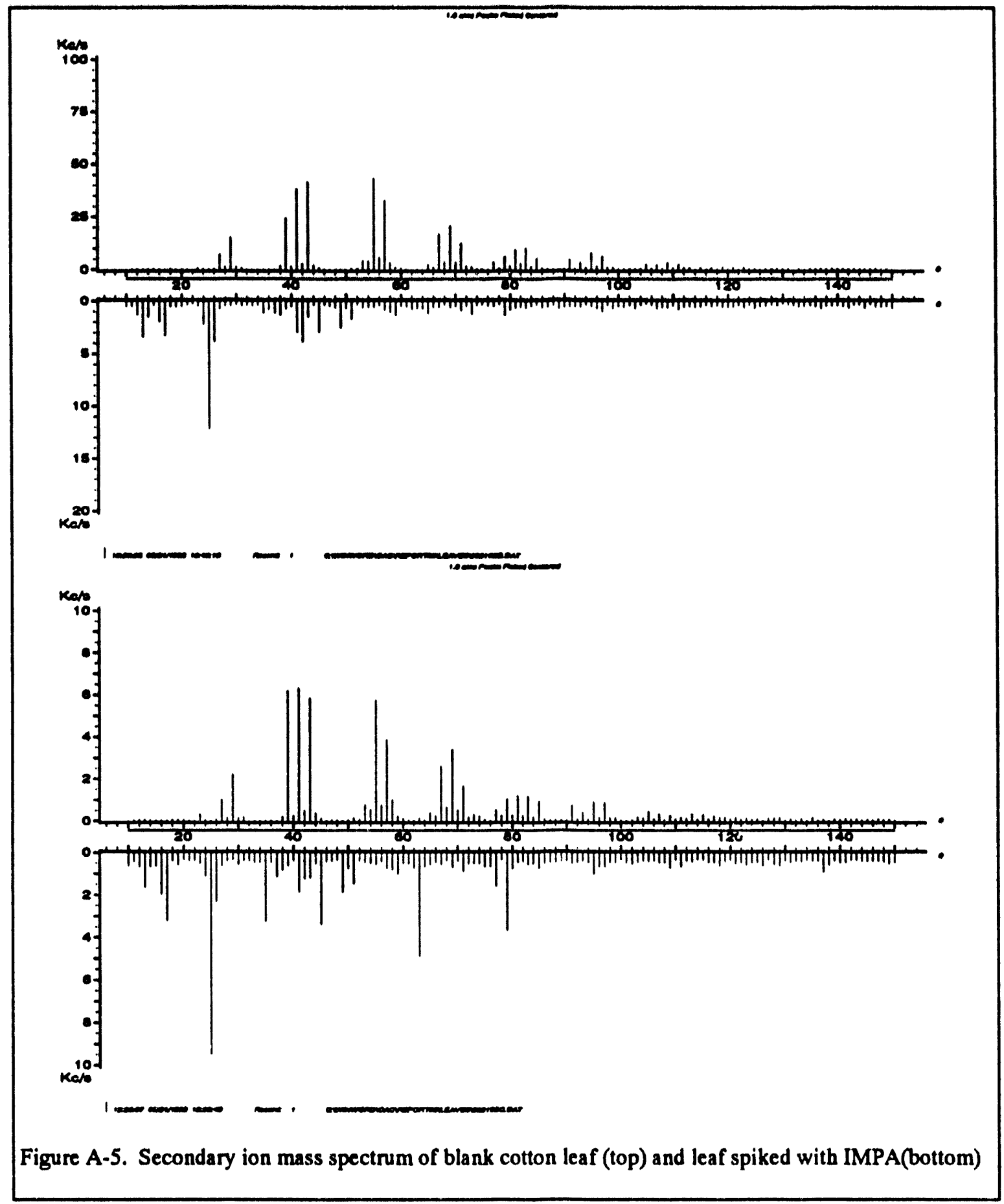




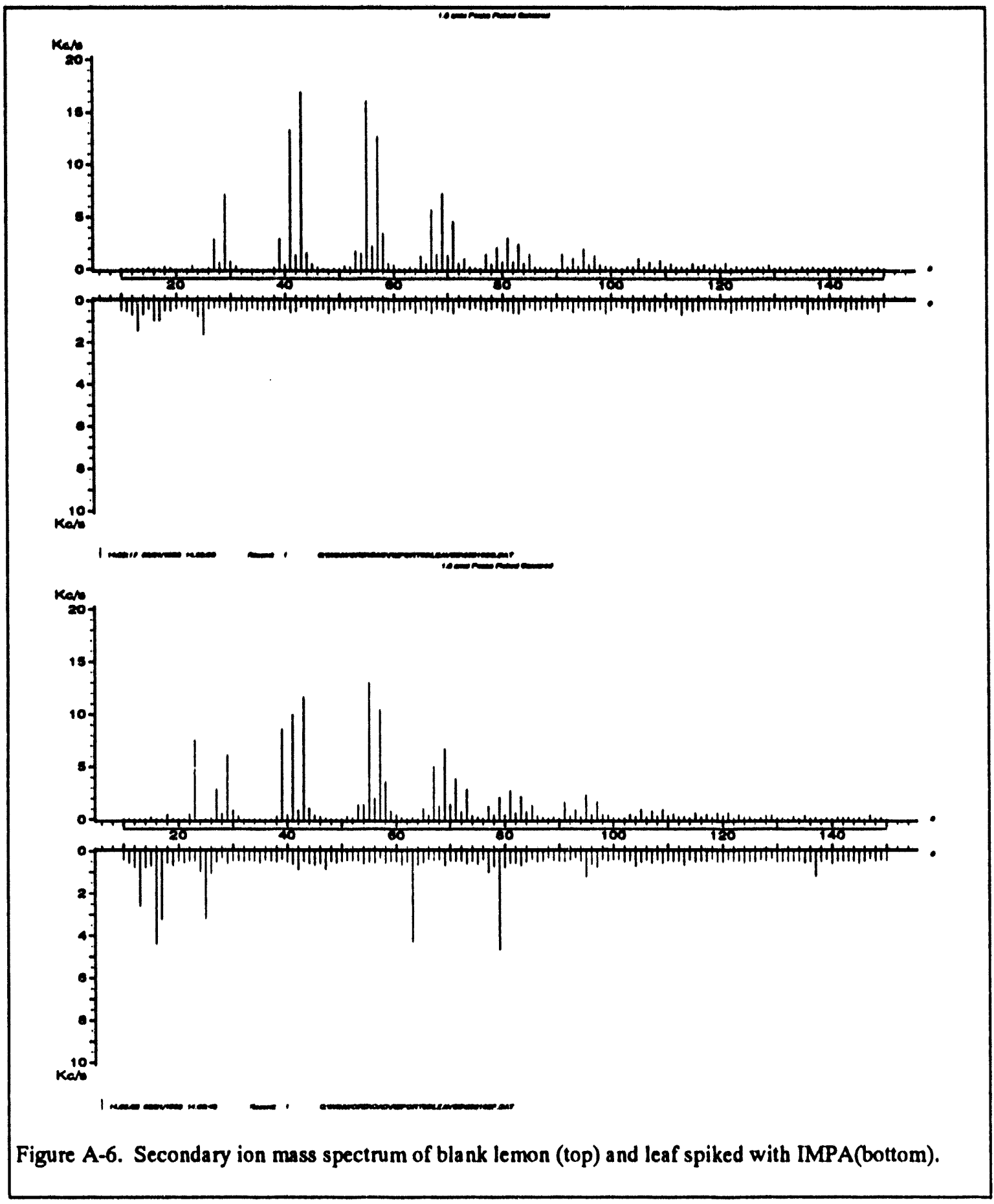




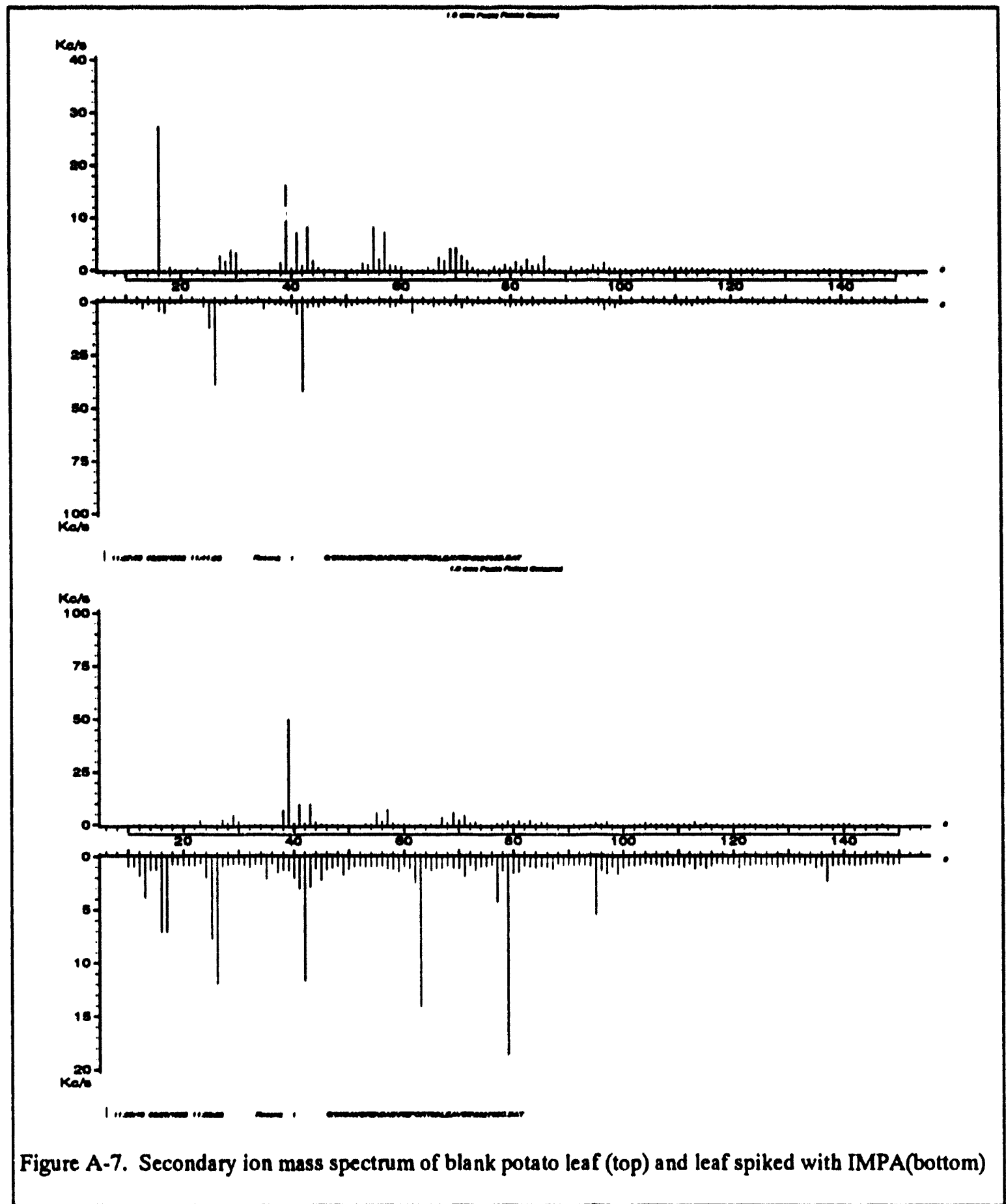




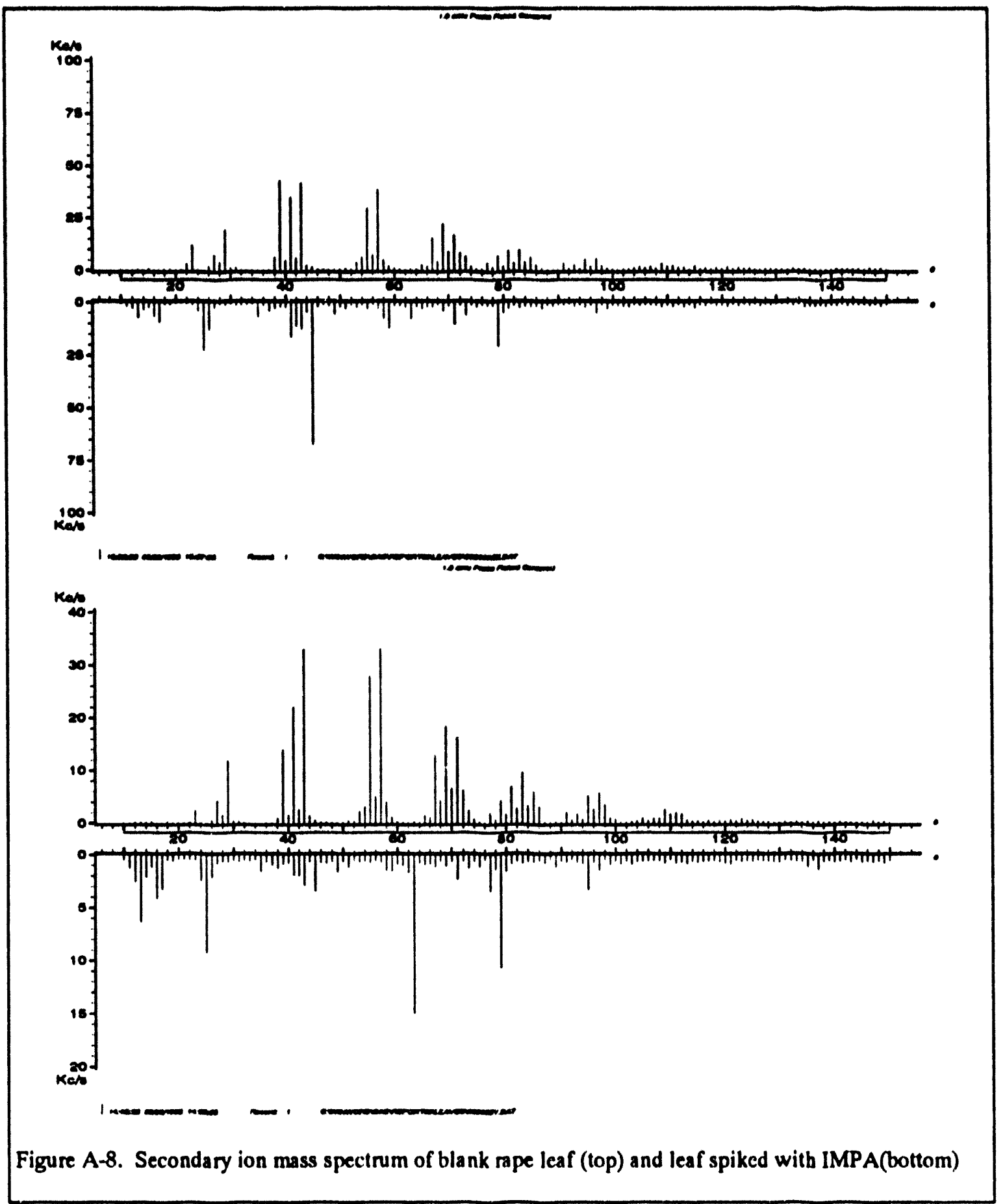


This Page Intentionally Left Blank 


\section{Advantages and Potential Applications of MBSA}

\section{Advantages and Comparison to Other Methods}

The MBSA instrument is intended to augment the current cadre of techniques and methods used to detect trace quantities of chemicals, not to compete with them. As such it is focused on detecting and identifying chemicals with low volatilities that are present primarily on the surface of the sample. This will enable the detection of chemicals not currently amerable to detection by techniques such as Gas Chromatography and Gas Chromatography Mass Spectrometry which rely on volatilizing the sample, and thus are very dependent on the sample volatility. For samples in which the target analyte resides primarily on the surface of the sample, either because of its chemical nature or because of the initial deposition mechanism, MBSA bas a distinct advantage. With MBSA only the top few molecular layers of the surface are sampled, and for these types of samples the surface concentration is very high relative to the bulk of the sample, and thus detection sensitivity is significantly increased. In addition to sample volatility and surface concentration advantages, MBSA technology accepts a very wide range of sample types and can analyze these without any prior chemical treatment, separation, or extraction. The molecular primary beam gun and pulsed extraction sample neutralization components are unique, and distinguish MBSA from standard Secondary lon Mass Spectrometry. No other method of sample neutralization is as widely applicable and easily performed as pulsed extraction sample neutralization, and there are no heavy molecular ion guns available commercially. These unique features are the enabling technology that make MBSA a viable and useful addition to the cadre of analytical tools heing applied to detection of trace quantities of chemicals on solids.

GC/MS The major differences between GC/MS and MBSA are that 1) MBSA is not reliant on sample volatility and can detect non-volatile compounds, 2) MBSA requires no chemical extraction, separation, concentration or derivatization of the sample prior to analysis, 3) MBSA generates no waste solvents or extracts that require disposal, and 4) MBSA is macroscopically non-destructive, altering only the top few molecular layers of the sample and permitting additional tests to be performed on the sample after MBSA.

Ion Mobility Spectrometry (IMS) The major difference between IMS and MBSA are the same as those described for GS/MS. One additional difference is that MBSA is a mass spectral technique, and accordingly, has much higher specificity than does IMS.

Standard SIMS While MBSA is a form of Secondary lon Mass Spectrometry, there are significant differences. MBSA uses a molecular primary beam, produced with a ceramic anion source for ruggedness and lower pumping load, which gives enhanced sensitivity (X10) over conventional SIMS ion guns. MBSA uses pulsed positive/negative secondary ion extraction - no electron flooding is needed to prevent sample charging resulting in less sample damage, operational simplicity, and lower cost. In addition, pulsed secondary ion extraction can give both positive and negative secondary ion spectra in each analysis. MBSA will utilize the Ion Trap Mass Spectrometer with MS/MS capability providing high specificity reduced chemical noise and reduced false positives. Coupled together the ceramic anion source + pulsed secondary ion extraction + ion trap makes benchtop size possible.

\section{Potential Applications}

\section{DOE and DOD}

Instrumentation and methods developed as part of the Molecular Beam Surface Analysis project can be applied to the detection and identification of a wide range of chemicals. Thus uses could include a broad spectrum, ranging from on-site challenge inspections by the CWC International Inspectorate and analysis at field locations (office building environment) of covertly obtained field samples by intelligence operations, to standard laboratory analysis of samples obtained by any means. This technology can also be applied to the detection and identification of 
hazardous and toxic wastes, and thus may find application at DOE sites, and in other areas of the world where the U. S. government is assisting in the surveying and remediation of polluted and contaminated areas. The speed of the analysis coupled with the fact that no sample preparation is needed make this a fast and inexpensive survey tool. The following summarizes the advantages offered by MBSA to potential areas of application within the United States government. Following this summary are possible applications within the broader scope of United States commerce and industry.

Treaty Verification Field support (analysis) for inspection teams in cooperative environments.

Detection of CW precursors and decomposition products: Provides opportunity to quickly prescreen samples and make in-the-field decisions on further sampling.

Sampling is simple; involves retrieving a sample (like leaves, rocks, building materials, swipes), cutting it to suitable size (1/2 inch) and inserting it directly into the instrument. No sample preparation, chromatography, or extraction chemistry is required.

Analysis is rapid, taking $\sim 5$ minutes.

Sample is not destroyed in the analysis and can be kept for further/altemate testing, providing additional evidence to reduce false detection rate.

Device is transportable ( $<200 \mathrm{lbs}$ ), could be operated in the field (in a van), or set up on a berich top (or desk).

Examples of potential uses are surveys of foreign sites performed under treaties, surveys of US DOD/DOE sites, surveys of foreign sites being surveyed for contamination and remediation as part of international cooperative agreements.

Challenge Inspections Field support for challenge inspections under hostile conditions, time constraints critical.

Detection of CW precursors and decomposition products, detection of HE feedstocks, agents, decomposition products: Provides opportunity to quickly prescreen samples and make in-the-field decisions on further sampling.

Fast survey analysis for a wide variety of products.

Requires no sample preparation, no chemical extraction, no generation of wastes.

Samples are preserved.

Wide range of samples can be accommodated (essentially anything solid that can be cut to $\sim 1 / 2$ diameter).

No special sample handling or preservation protocols are required (as in the case of water and soil samples).

Nuclear Non-proliferation Detection of HE feedstocks, agents, decomposition products; detection of chemicals used in nuclear materials processing (e.g. tributyl phosphate).

MBSA is an excellent method for identifying polymers typical of those used as fillers and for shaping.

MBSA has been shown to be highly sensitive to chemicals such as tributyl phosphate used in nuclear fuel processing. 
The same advantages listed for Treaty Verification and Challenge Inspections apply to this application.

Intelligence Analysis of samples collected in a non-cooperative environment.

Instrument could be operated in an office environment, no need for preparatory chemicals, waste disposal, sample preparation.

Samples can be screened prior to shipment to formal analytical laboratory, or samples can be surveyed and then destroyed.

The same advantages described for Treaty Verification and Challenge Inspections also apply to this application.

Routine Analysis Piggyback analysis on existing sample protocols.

Since the technique is non-destructive (to the bulk of the sample) the samples could be analyzed first with this technique before going on for conventional analyses.

Alternately, a small portion (only $\sim 1 / 8$ inch square) of a sample could be used for this analyses, while the bulk went on for other analyses.

The wide range of sample types that this technique accommodates would support many of the samples already collected.

The analysis is fast and inexpensive and could be added to existing protocols with minimal cost impact, while adding another dimension to the overall characterization of the sample.

Remediation/Characterization Detection of hazardous/toxic materials at fabrication/storage/waste sites.

All of the advantages listed for the previous applications would apply.

Examples of potential uses are surveys of US DOD/DOE sites undergoing site remediation/characterization, surveys of foreign sites being surveyed for contamination and remediation as part of international cooperative agreements.

\section{General Applications within U. S. Industry and Commerce}

Agriculture Direct detection of pesticides, herbicides, fungicides, and other chemicals used in the agriculture industry on plant surfaces.

Surveys of imported vegetables, fruits, or general vegetation (flowers/shrubs/trees etc.).

Field surveys for support of crop management (pesticide/fungicide application).

Surveys of stored fruits/vegetables to support storage management (anti-sprouting agents, fungicides).

Applied research of the environmental fate/pathways of pesticides/herbicides/fungicides.

Chemistry Analysis of low volatility compounds at trace ccncentrations.

Determination of molecular structure during development of new low volatility compounds.

Determination of chemical environment at the surface of materials. 
Envirommental Direct detection of trace concentration of hazardous/toxic materials on the surfaces of samples.

Surveys of hazardous waste sites.

Surveys of buildings suffering "sick building" syndrome.

Monitoring of controlled waste storage/destruction facility sites.

Industrial Processes Detection and monitoring of the chemical composition of the surface of materials before, during and after processing.

Supports process control and quality control.

Supports product and process development.

\section{Further Information and Contacts}

Further information on Molecular Beam Surface Analysis can be obtained from:
A. D. Appelbans (208) 526-0862
FAX (208) 526-8541
G. S. Groenewold (208) 526-2803
FAX (208) 526-8541
J. E. Delmore (208) 526-2820
FAX (208) 526-8541

Idaho National Engineering Laboratory

PO Box 1625

Idaho Falls, ID 83415-2208 

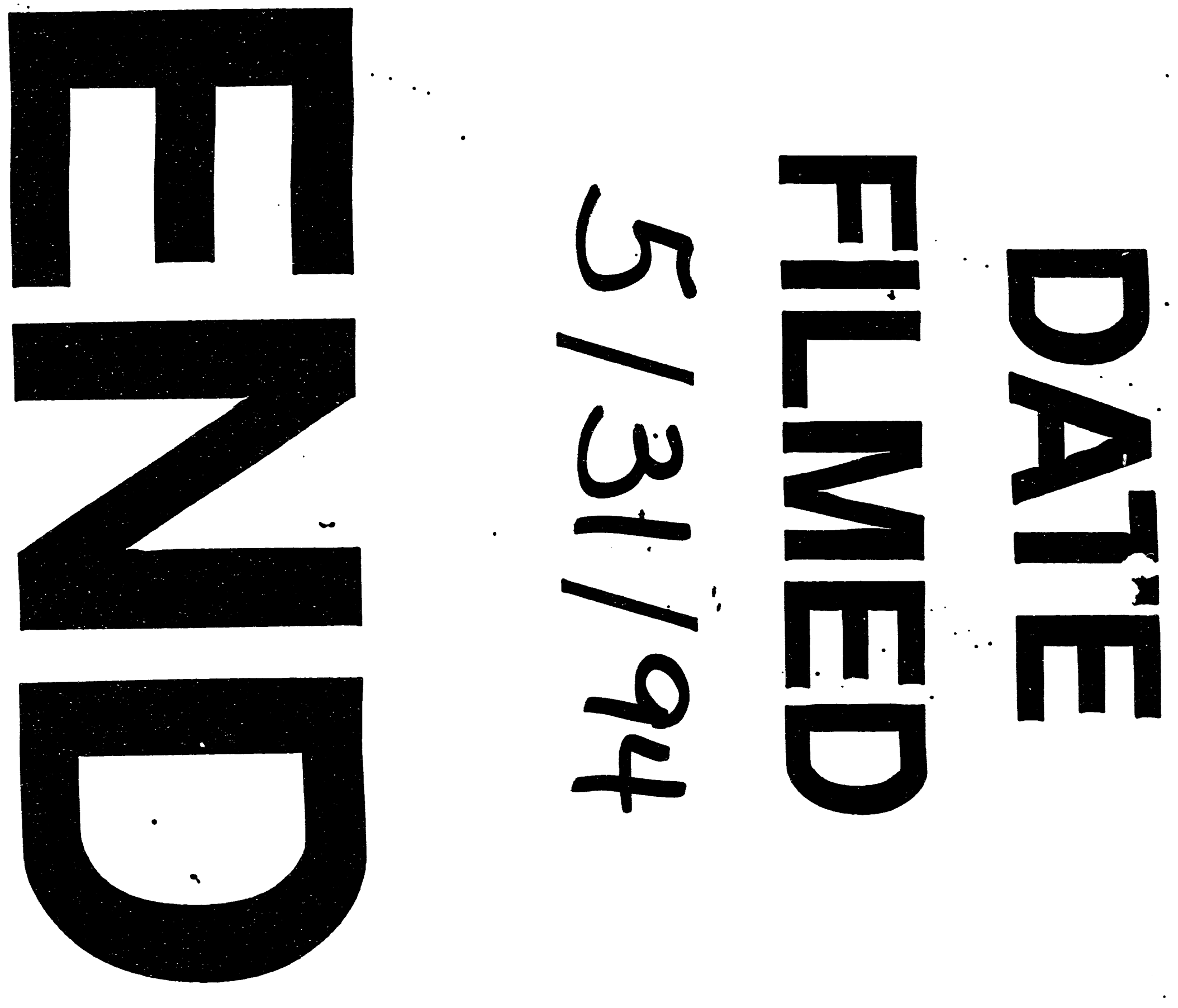
